Prepared in cooperation with the Central Valley Project-Bureau of Reclamation and U.S. Fish and Wildlife Service

\title{
An Evaluation of the Efficacy of Using Environmental DNA (eDNA) to Detect Giant Gartersnakes (Thamnophis gigas)
}

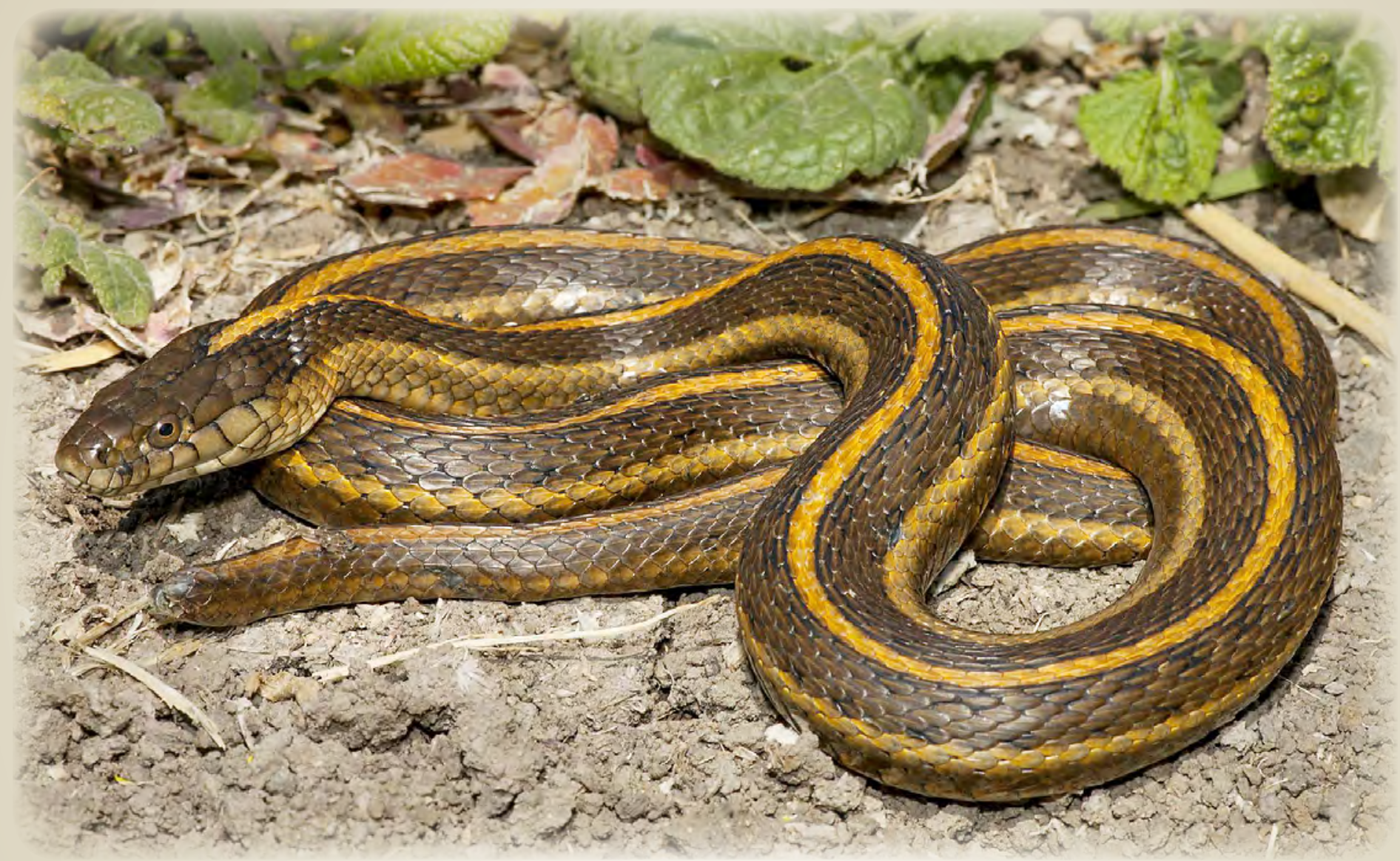

Open-File Report 2017-1123

U.S. Department of the Interior

U.S. Geological Survey 
Cover: Photograph showing giant gartersnake (Thamnophis gigas) in the Natomas Basin, California, 2015. Photograph by Chris Murphy, U.S. Geological Survey. 


\section{An Evaluation of the Efficacy of Using Environmental DNA (eDNA) to Detect Giant Gartersnakes (Thamnophis gigas)}

By Brian J. Halstead, Dustin A. Wood, Lizabeth Bowen, Shannon C. Waters, Amy G. Vandergast, Julia S. Ersan, Shannon M. Skalos, and Michael L. Casazza

Prepared in cooperation with the Central Valley Project-Bureau of Reclamation and U.S. Fish and Wildlife Service

Open-File Report 2017-1123

U.S. Department of the Interior

U.S. Geological Survey 


\section{U.S. Department of the Interior \\ RYAN K. ZINKE, Secretary}

\section{U.S. Geological Survey \\ William H. Werkheiser, Acting Director}

U.S. Geological Survey, Reston, Virginia: 2017

For more information on the USGS - the Federal source for science about the Earth,

its natural and living resources, natural hazards, and the environment-visit

https://www.usgs.gov or call 1-888-ASK-USGS (1-888-275-8747).

For an overview of USGS information products, including maps, imagery, and publications, visit https://store.usgs.gov. The findings and conclusions in this article are those of the author(s) and do not necessarily represent
the views of the U.S. Fish and Wildlife Service.

Any use of trade, firm, or product names is for descriptive purposes only and does not imply endorsement by the U.S. Government.

Although this information product, for the most part, is in the public domain, it also may contain copyrighted materials as noted in the text. Permission to reproduce copyrighted items must be secured from the copyright owner.

Suggested citation:

Halstead, B.J., Wood, D.A, Bowen, Lizabeth, Waters, Shannon, Vandergast, A.G., Ersan, J.S.M., Skalos, S.M., and Casazza, M.L., 2017, An evaluation of the efficacy of using environmental DNA (eDNA) to detect giant gartersnakes (Thamnophis gigas): U.S. Geological Survey Open-File Report 2017-1123, 41 p., https://doi.org/10.3133/ofr20171123.

ISSN 2331-1258 (online) 


\section{Contents}

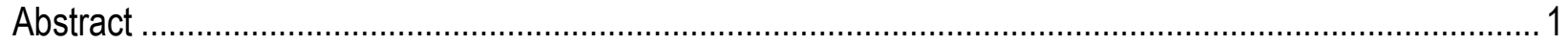

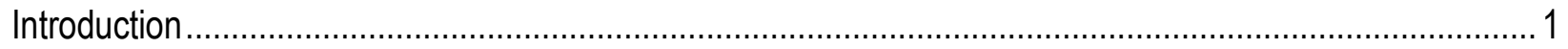

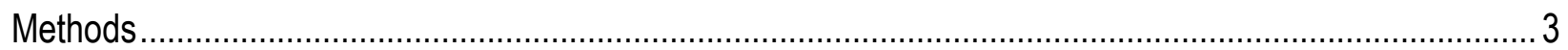

Laboratory Experiment............................................................................................................

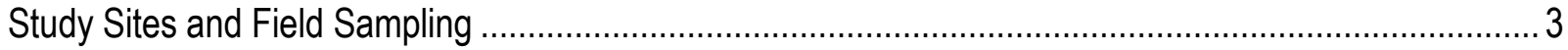

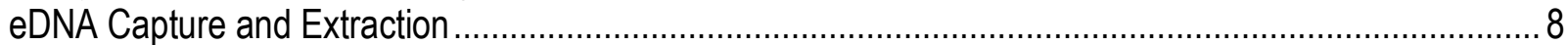

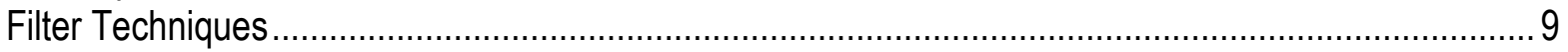

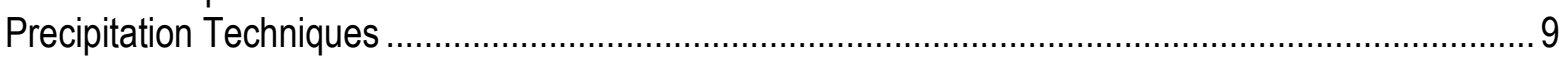

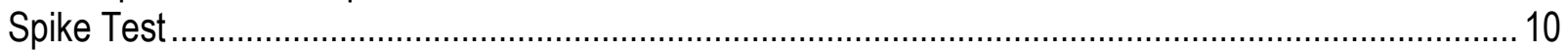

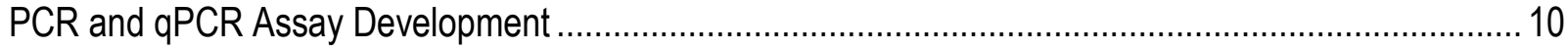

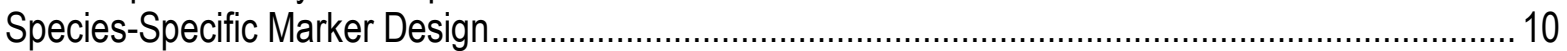

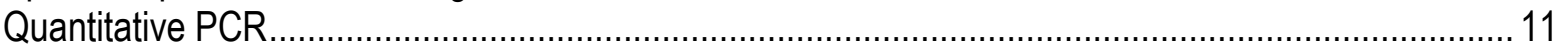

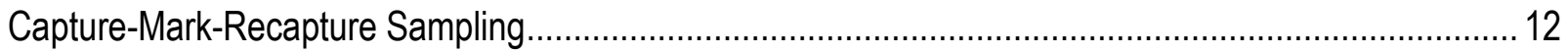

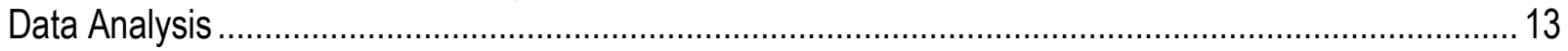

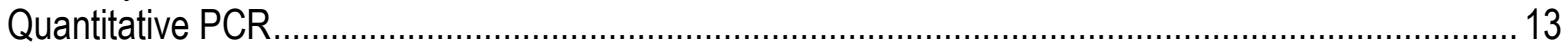

Capture-Mark-Recapture ………........................................................................................ 13

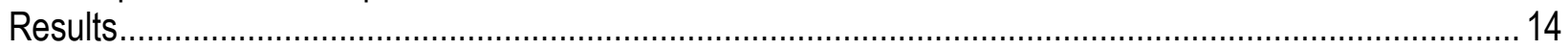

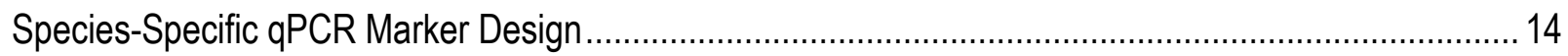

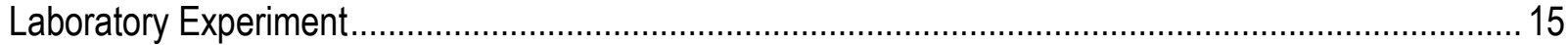

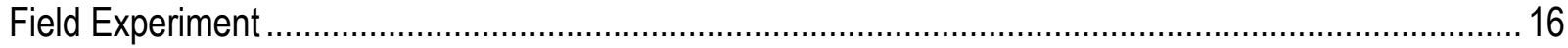

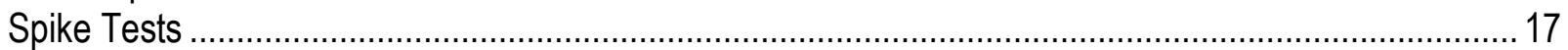

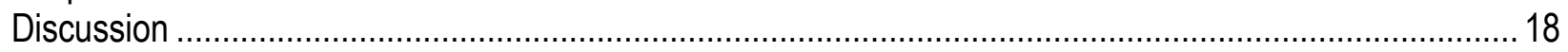

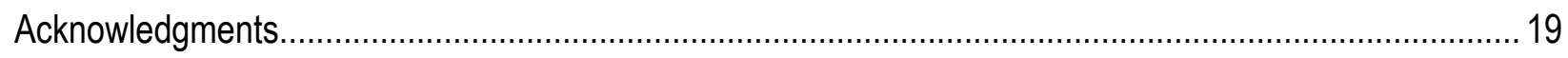

References Cited ................................................................................................................ 19

Appendix 1. Sequence Alignment of Thamnophis gigas and Closely Related and (or) Potentially

Sympatric Snake Species for the Nicotinamide Adenine Dinucleotide (Reduced Form)(NADH)

Dehydrogenase Subunit 4 (ND4) and Primers/Probe Set Used in Quantitative Polymerase

Chain Reaction (qPCR) Assays.

Appendix 2. Sequence Alignment of Nerodia fasciata and Potentially Sympatric Snake Species for the Nicotinamide Adenine Dinucleotide (Reduced Form) (NADH) Dehydrogenase Subunit 4 (ND4) and Primers/Probe Set for Use in Quantitative Polymerase Chain Reaction (qPCR) Assays, in the Central Valley, California .....

Appendix 3. Sequence Alignment of Thamnophis gigas and Potentially Sympatric Snake Species for the Cytochrome b (Cytb) Gene and Primers/Probe Sets for Use in Quantitative Polymerase Chain Reaction (qPCR) Assays, in the Central Valley, California.

Appendix 4. Sequence Alignment of Thamnophis gigas and Nerodia fasciata and Potentially Sympatric Snake Species for the Nicotinamide Adenine Dinucleotide (Reduced Form) (NADH) Dehydrogenase Subunit 2 (ND2) and Primers/Probe Set for Use in Quantitative Polymerase Chain Reaction (qPCR) Assays, in the Central Valley, California 


\section{Figures}

Figure 1. Location of the Betts-Kismat-Silva tract in the Natomas Basin, including sample, giant gartersnake (GGS; Thamnophis gigas) capture, and trap locations used for evaluation of environmental DNA (eDNA) to detect giant gartersnakes in the Sacramento Valley, California, 2015 ......... 4

Figure 2. Location of the Glenn-Colusa Irrigation District canal at Colusa National Wildlife Refuge, including sample, giant gartersnake (GGS; Thamnophis gigas) capture, and trap locations used for evaluation of environmental DNA (eDNA) to detect giant gartersnakes in the Sacramento Valley, California, 2015

Figure 3. Location of East Pond at Colusa National Wildlife Refuge, including sample, giant gartersnake (GGS; Thamnophis gigas) capture, and trap locations used for evaluation of environmental DNA (eDNA) to detect giant gartersnakes in the Sacramento Valley, California, 2015.

Figure 4. Experimental design used to collect 120 field water samples from two locations with known giant gartersnake (Thamnophis gigas) populations across two habitat types within the 2015 field season (May, July, and October).....

Figure 5. Experimental workflow used to evaluate different capture, extraction, and assay techniques for environmental deoxyribonucleic acid (eDNA) detection

\section{Tables}

Table 1. Primer and probe sets for ND4, ND2, and CytB gene regions for giant gartersnake (Thamnophis gigas) and banded watersnake (Nerodia fasciata)....

Table 2. Detection results using the pre-qPCR assay for giant gartersnakes from the different treatments (i.e. DNA source) from the laboratory trials

Tablel 3. qPCR results from field collected water samples by habitat type and sample period using pre-qPCR assay

Table 4. qPCR results from precipitation capture and extraction techniques run with standard and pre-qPCR assays (N, number of samples; rxns, number of reactions)

Table 5. Spiked DNA test results to evaluate the efficiency of filtration and capture techniques 


\section{Conversion Factors}

International System of Units to Inch/Pound

\begin{tabular}{lcl}
\hline & Multiply & By \\
\hline & Length & \\
\hline micrometer, micron $(\mu \mathrm{m})$ & $3.937 \times 10^{-5}$ & inch (in.) \\
millimeter $(\mathrm{mm})$ & 0.03937 & inch (in.) \\
centimeter $(\mathrm{cm})$ & 0.3937 & inch (in.) \\
\hline & Mass & \\
\hline nanogram $(\mathrm{ng})$ & $3.527 \times 10^{-11}$ & ounce, avoirdupois (oz) \\
milligram $(\mathrm{mg})$ & $3.527 \times 10^{-5}$ & ounce, avoirdupois (oz) \\
gram $(\mathrm{g})$ & 0.03527 & ounce, avoirdupois (oz) \\
\hline & Volume & \\
\hline microliter $(\mu \mathrm{L})$ & 0.0002029 & teaspoon (tsp.) \\
milliliter $(\mathrm{mL})$ & 0.2029 & teaspoon (tsp.) \\
liter $(\mathrm{L})$ & 4.167 & cup, legal (c) \\
liter $(\mathrm{L})$ & 2.114 & pint, liquid (pt.) \\
liter $(\mathrm{L})$ & 1.057 & quart, liquid (qt.) \\
liter $(\mathrm{L})$ & 0.2642 & gallon, liquid (gal.) \\
\hline
\end{tabular}

Temperature in degrees Celsius $\left({ }^{\circ} \mathrm{C}\right)$ may be converted to degrees Fahrenheit $\left({ }^{\circ} \mathrm{F}\right)$ as ${ }^{\circ} \mathrm{F}=\left(1.8 \times{ }^{\circ} \mathrm{C}\right)+32$.

\section{Datum}

Horizontal coordinate information is referenced to the Universal Transverse Mercator, North American Datum of 1927 (NAD 27). 


\section{Abbreviations}

$\begin{array}{ll}\text { 6-FAM } & \text { 6-Carboxyflourescein } \\ \text { ABI } & \text { Applied Biosystems, Inc. } \\ \text { BKS } & \text { Betts-Kismat-Silva } \\ \text { Cq }_{\text {q }} & \text { quantification cycle } \\ \text { CI } & \text { credible interval } \\ \text { CMR } & \text { capture-mark-recapture } \\ \text { Cytb } & \text { Cytochrome b } \\ \text { DI } & \text { deionized } \\ \text { DNA } & \text { deoxyribonucleic acid } \\ \text { eDNA } & \text { environmental DNA } \\ \text { GCID } & \text { Glenn-Colusa Irrigation District } \\ \text { gDNA } & \text { genomic DNA } \\ \text { IBFQ } & \text { 3lowa Black }{ }^{\circledR} \text { FQ probe quencher } \\ \text { IDT } & \text { Integrated DNA Technologies } \\ \text { IDT_LNA } & \text { Integrated DNA Technologies dual-labeled DNA probe } \\ \text { JAGS } & \text { Just Another Gibbs Sampler } \\ \text { M } & \text { molar } \\ \text { MCMC } & \text { Markov chain Monte Carlo } \\ \text { MJ } & \text { MJ Research, Inc. } \\ \text { mtDNA } & \text { mitochondrial deoxyribonucleic acid } \\ \text { NADH } & \text { nicotinamide adenine dinucleotide (reduced form) } \\ \text { ND2 } & \text { NADH dehydrogenase subunit 2 } \\ \text { ND4 } & \text { NADH dehydrogenase subunit 4 } \\ \text { nM } & \text { nanomolar } \\ \text { NWR } & \text { National Wildlife Refuge } \\ \text { PCR } & \text { polymerase chain reaction } \\ \text { qPCR } & \text { quantitative polymerase chain reaction } \\ \text { RSC } & \text { relative standard curve } \\ \text { SA } & \text { Short Amplicon } \\ \text { SVL } & \text { snout-vent length } \\ \text { USGS } & \text { U.S. Geological Survey } \\ \text { UV } & \text { ultraviolet } \\ & \end{array}$




\title{
An Evaluation of the Efficacy of using Environmental DNA (eDNA) to Detect Giant Gartersnakes (Thamnophis gigas)
}

By Brian J. Halstead, Dustin A. Wood, Lizabeth Bowen, Shannon C. Waters, Amy G. Vandergast, Julia S. Ersan, Shannon M. Skalos, and Michael L. Casazza

\begin{abstract}
Detecting populations of rare or cryptic species is essential for their conservation. For species like giant gartersnakes (Thamnophis gigas), conventional survey methods can be expensive and inefficient. These sampling difficulties might be overcome by modern techniques that detect deoxyribonucleic acid (DNA) shed by organisms into the environment (eDNA). We evaluated the efficacy of detecting giant gartersnake eDNA in water samples from the laboratory and at locations with known giant gartersnake populations in the Sacramento Valley of California, and failed to detect giant gartersnake DNA in most laboratory and all field samples. Aspects of giant gartersnake biology-such as highly keratinized skin and spending extensive time in the terrestrial environment, as well as hot, sunny, and turbid conditions in wetlands and canals of the Sacramento Valley-likely contributed to low detection probabilities. Although detection of eDNA shows promise under many conditions, further development is needed before sampling for eDNA is a viable option for detecting giant gartersnake populations.

\section{Introduction}

One of the most fundamental questions in ecology is, "Where do species occur?" Although seemingly simple on the surface, detecting animals in the field can be difficult. This is particularly true for rare species, which by definition have limited distributions, occur at low densities, or both. In addition to rarity, many species also are cryptic and difficult to detect, even when present in abundance. Giant gartersnakes (Thamnophis gigas) embody all these characteristics that create difficulties for detection.
\end{abstract}


Giant gartersnakes are listed as threatened under the U.S. and California Endangered Species Acts (California Fish and Game Commission, 1971; U.S. Fish and Wildlife Service, 1993). Although giant gartersnakes have lost more than 90 percent of their historical wetland habitat and have been nearly extirpated from the southern two-thirds of their range (U.S. Fish and Wildlife Service, 1993, 1999), they persist in regions of the Sacramento Valley where rice fields and their associated infrastructure of canals mimic the marsh conditions giant gartersnakes historically inhabited (Halstead and others, 2010). Their dependence on aquatic habitats means that giant gartersnakes are affected by water management decisions, but avoiding potential negative effects of these decisions is hampered by imperfect knowledge of where giant gartersnake populations occur. Snakes in general are difficult to find (Durso and others, 2011), but even among this group giant gartersnakes are notorious for being challenging to detect (Fitch, 1940; Halstead and others, 2011).

Because of their wariness and crypsis, trapping for giant gartersnakes is the standard sampling method for detecting the species. Although detection probabilities with trapping were low (0.12 [0.090.18]; Halstead and others, 2011), efforts to improve traps have increased their efficacy nearly sixfold (Halstead and others, 2013). Despite these improvements, trapping has several drawbacks for detecting the presence of giant gartersnakes. Traps work by detaining individuals until they can be removed from the trap, and individuals must be handled for release. The potential to harm individuals though trapping and processing requires that permits from State and Federal wildlife agencies be obtained to sample for giant gartersnakes. In addition to requiring permits, trapping is expensive, both because of the equipment necessary to trap snakes and because trapping is labor-intensive. Finally, much of the range of giant gartersnakes occurs on private property, and landowners can be reluctant to allow repeated visits by biologists and deployment of equipment on their land.

Deoxyribonucleic acid (DNA)-based detection methods offer possible alternatives to standard trapping techniques for giant gartersnakes that overcome some of the common difficulties associated with trapping. These methods may be able to enhance detection sensitivity and specificity of target wildlife non-invasively through the detection of DNA shed by organisms into water or onto soil. Environmental DNA (eDNA) sampling has been proven effective for detecting diverse organisms, including amphibians (Ficetola and others, 2008; DeJean and others, 2011; Goldberg and others, 2011; McKee, Calhoun, and others, 2015), fishes (DeJean and others, 2011; Jerde and others, 2011), and pathogens (Kirshtein and others, 2007). Recent developments also have indicated that eDNA can be effective for detecting invasive Burmese pythons (Python bivittatus) in Florida (Piaggio and others, 2014; Hunter and others, 2015). Whether eDNA can be an effective method for detecting giant gartersnakes from water samples, and under what conditions, is unknown.

Our primary objective was to examine whether detection of giant gartersnakes using eDNA was a viable and cost-effective method to document presence of the species, in comparison to trapping. We developed an eDNA assay specific to giant gartersnakes and assessed its ability to detect eDNA under controlled laboratory conditions and in the field from populations of known abundance. 


\section{Methods}

\section{Laboratory Experiment}

We first evaluated the ability to detect eDNA under controlled laboratory conditions in April and May 2015. In particular, we evaluated the ability to detect giant gartersnake DNA from three treatments: (1) a live snake (79 g), (2) a shed snakeskin (1.08 g), and (3) a fecal sample (1.4 g) placed in tanks initially containing $40 \mathrm{~L}$ of deionized water. We also ran a control tank during the laboratory experiment that contained only $40 \mathrm{~L}$ of deionized water. The live snake was placed in the tank for $24 \mathrm{~h}$ with a $5 \times$ $15 \times 30 \mathrm{~cm}$ piece of softwood lumber; other treatments were left in the water. From each tank, 1-L water samples were taken by submerging a 1-L Nalgene ${ }^{\mathrm{TM}}$ bottle in the tank just prior to the introduction of the sample (Day 0), and again after 1, 2, 3, 8, 16, and $21 \mathrm{~d}$. Samples were filtered for genomic DNA immediately after collection using a peristaltic pump connected to a vacuum flask fitted with a disposable filter funnel containing a $47-\mathrm{mm}$-diameter $0.45-\mu \mathrm{m}$ nitrocellulose filter. Filters were preserved in ethanol and refrigerated at $4{ }^{\circ} \mathrm{C}$ until they were shipped overnight to the U.S. Geological Survey (USGS) San Diego Field Station for DNA extraction.

\section{Study Sites and Field Sampling}

In addition to the laboratory experiment, we collected water samples in two habitat types (wetland and canal) at each of two sites (the BKS [Betts-Kismat-Silva] tract in the Natomas Basin and the Colusa National Wildlife Refuge [NWR] in the Colusa Basin; figs. 1-3) with known populations of giant gartersnakes. We collected three 1-L water samples in Nalgene ${ }^{\mathrm{TM}}$ bottles at each of three locations at the four sites at three different times (figs. 1-4): (1) May 20, 2015; (2) July 22, 2015; and (3) October 1,2015 . We also collected an additional 1-L water sample from each location during the October 1 , 2015, sampling occasion, for a total of 120 samples. To each sample, we immediately added $1 \mathrm{~mL}$ of 3molar (M) sodium acetate and $33 \mathrm{~mL}$ of 95 percent ethanol (Hunter and others, 2015), and placed each sample in a cooler containing ice. As a negative control, we poured $1 \mathrm{~L}$ of store-bought deionized water into a 1-L Nalgene ${ }^{\mathrm{TM}}$ bottle and added sodium acetate and ethanol using the same procedure as for the grab samples at each sampling location and date, for a total of 36 negative controls. We transported all samples and controls to the U.S. Geological Survey Dixon Field Station within $4 \mathrm{~h}$ of collection, where samples were stored in a non-self-defrosting freezer $\left(-20^{\circ} \mathrm{C}\right)$ until capture and extraction methods were performed. 


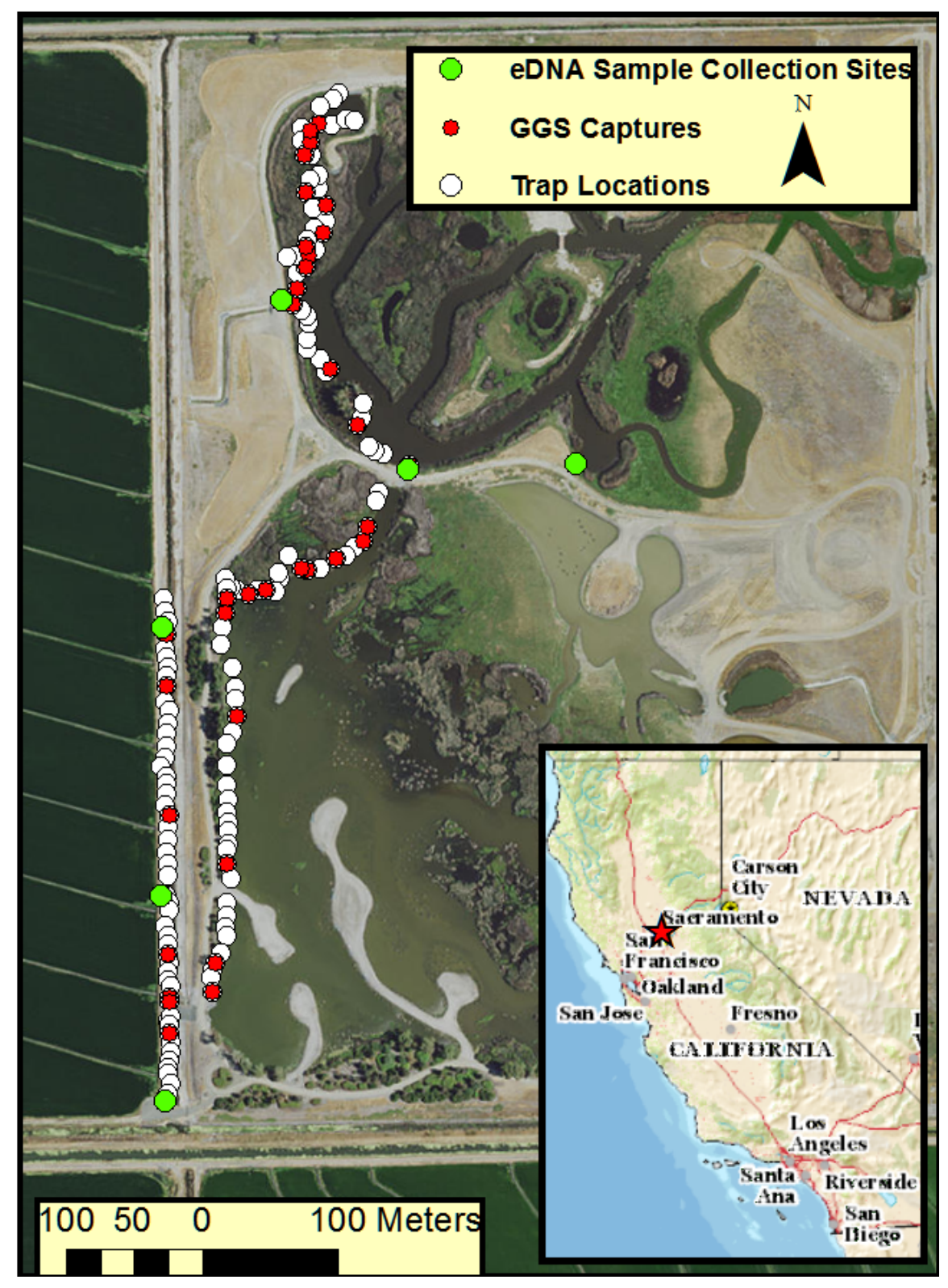

Figure 1. Location of the Betts-Kismat-Silva tract in the Natomas Basin, including sample, giant gartersnake (GGS; Thamnophis gigas) capture, and trap locations used for evaluation of environmental DNA (eDNA) to detect giant gartersnakes in the Sacramento Valley, California, 2015. Horizontal coordinate information is referenced to the Universal Transverse Mercator, North American Datum of 1927 (NAD 27). 


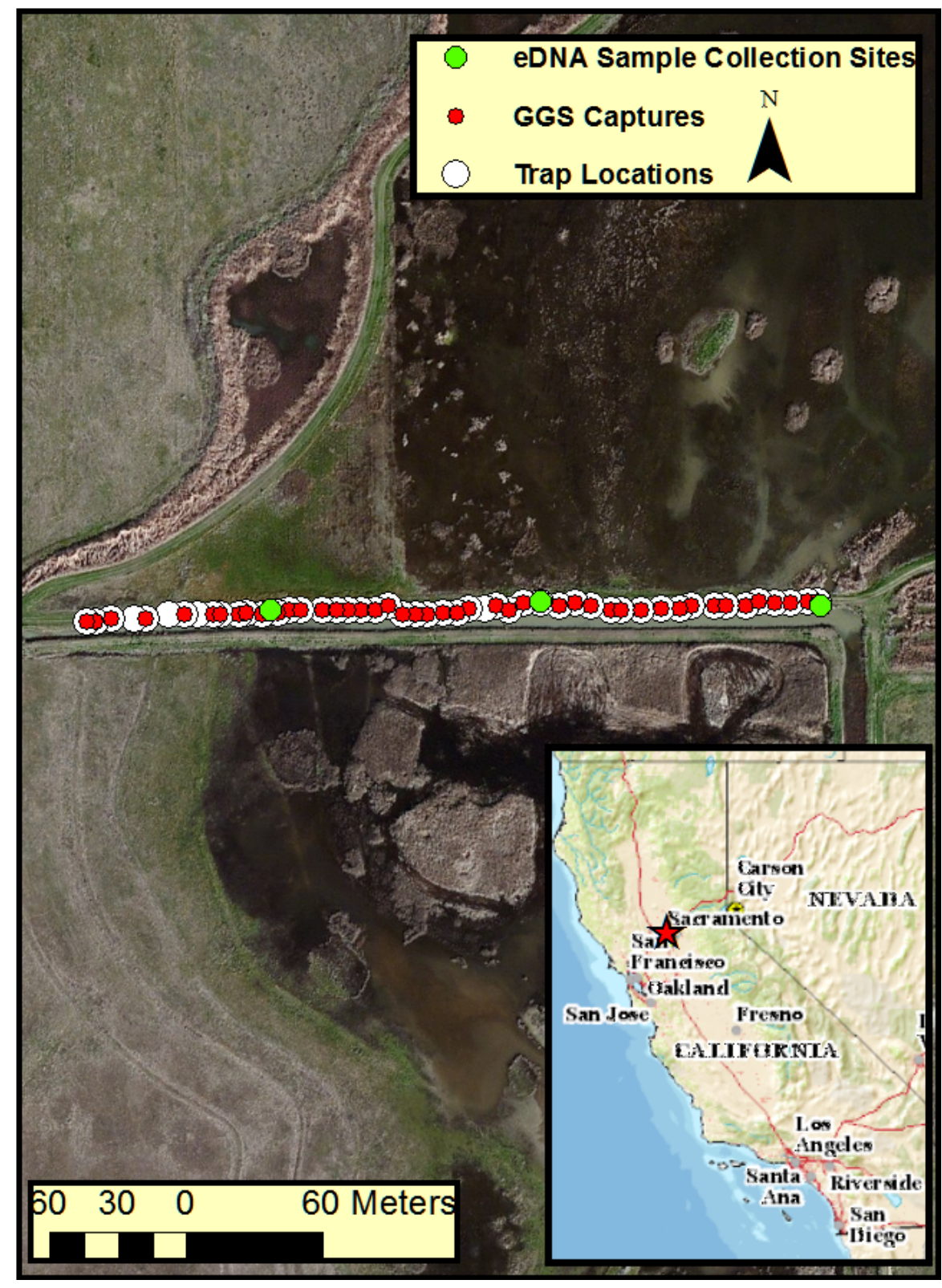

Figure 2. Location of the Glenn-Colusa Irrigation District canal at Colusa National Wildlife Refuge, including sample, giant gartersnake (GGS; Thamnophis gigas) capture, and trap locations used for evaluation of environmental DNA (eDNA) to detect giant gartersnakes in the Sacramento Valley, California, 2015. Horizontal coordinate information is referenced to the Universal Transverse Mercator, North American Datum of 1927 (NAD 27). 


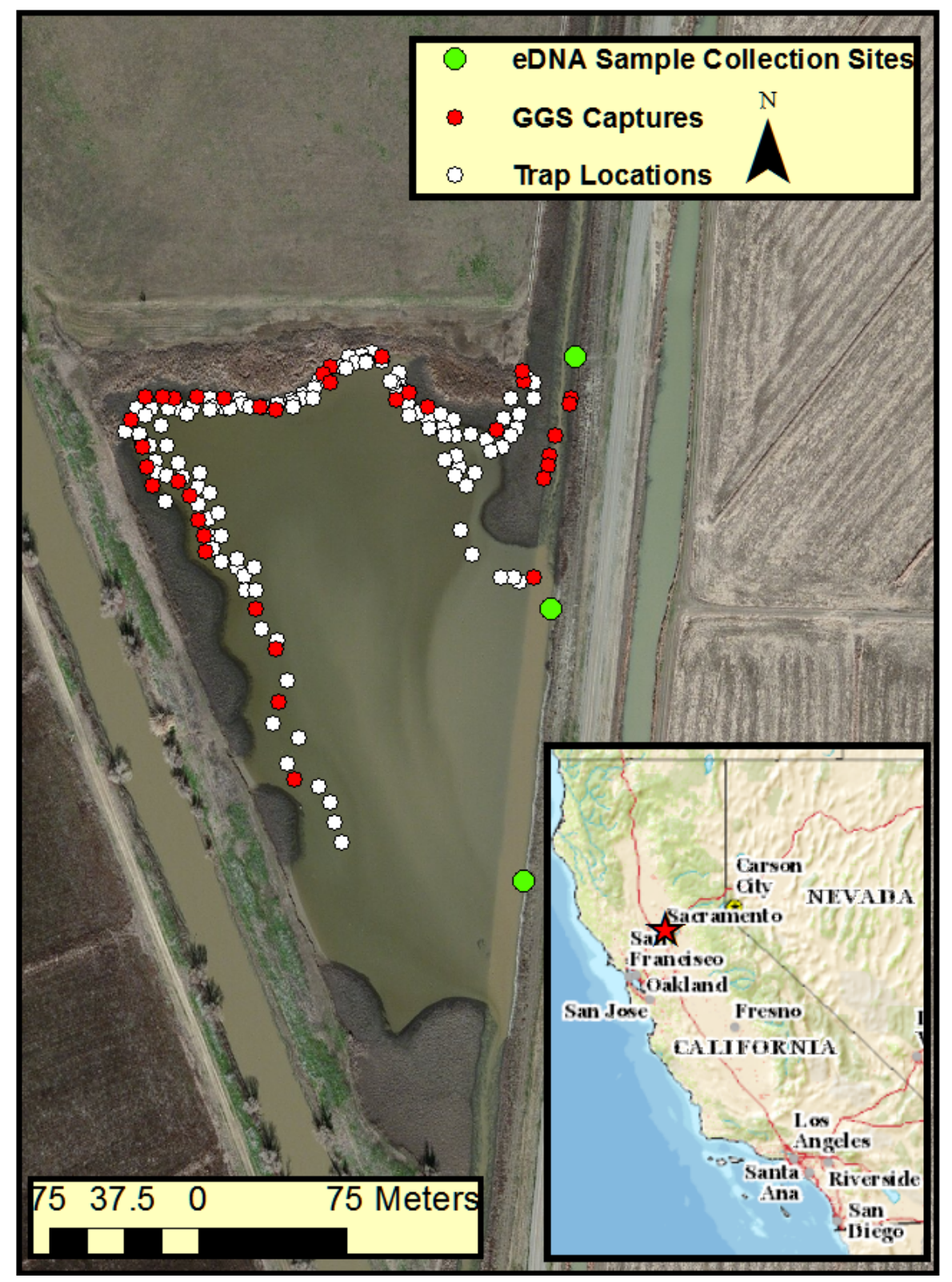

Figure 3. Location of East Pond at Colusa National Wildlife Refuge, including sample, giant gartersnake (GGS; Thamnophis gigas) capture, and trap locations used for evaluation of environmental DNA (eDNA) to detect giant gartersnakes in the Sacramento Valley, California, 2015. Horizontal coordinate information is referenced to the Universal Transverse Mercator, North American Datum of 1927 (NAD 27). 

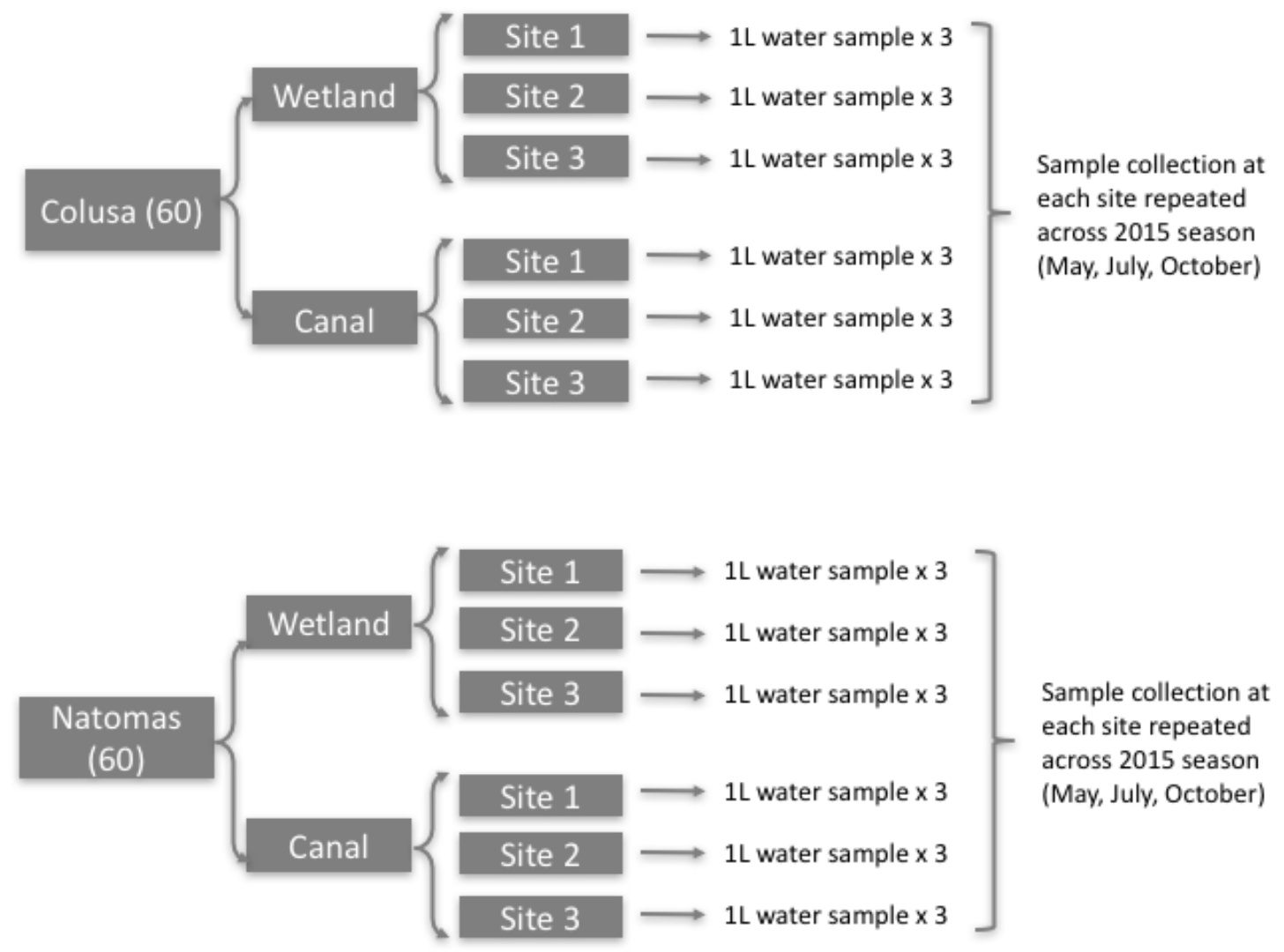

Figure 4. Experimental design used to collect 120 field water samples from two locations with known giant gartersnake (Thamnophis gigas) populations across two habitat types within the 2015 field season (May, July, and October). In October, we collected four 1-liter water samples (instead of three) from each site. 


\section{eDNA Capture and Extraction}

On the basis of literature searches (for example, Deiner and others, 2015; Goldberg and others, 2015; Goldberg and others, 2016; Hunter and others, 2015) and communications with eDNA specialists, we developed a workflow for capture and extraction of eDNA from water samples (fig. 5). However, we also evaluated several alternative capture and extraction methods and polymerase chain reaction (PCR) steps (for example, PCR inhibitor kits) that have been used in eDNA applications (for example, Piaggio and others, 2014, McKee, Spear, and others, 2015, Spens and others, 2017) for comparison to our preferred workflow.

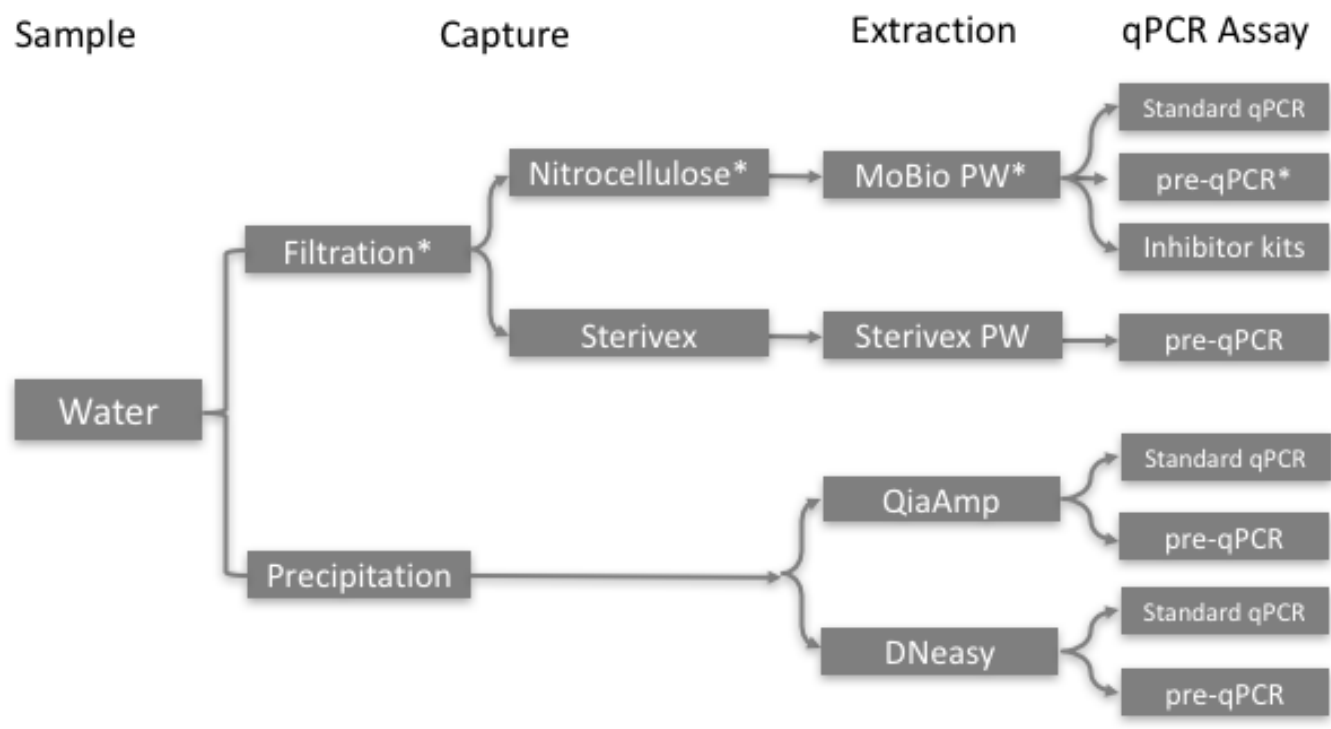

Figure 5. Experimental workflow used to evaluate different capture, extraction, and assay techniques for environmental deoxyribonucleic acid (eDNA) detection. The asterisk indicates the preferred workflow for evaluating most of our laboratory and field samples. 


\section{Filter Techniques}

We captured genomic DNA (gDNA) from laboratory and field-collected water samples using 47-mm-diameter nitrocellulose filters $(0.45 \mu \mathrm{m})$. Prior to filtration, water samples were thawed overnight at room temperature. Because of long filtration times and the necessity of using multiple filters for most field samples, we used a reusable polysulfone filter holder with funnel (Thermo Scientific, Waltham, Massachusetts), and replaced the nitrocellulose filter membranes when the flow rate through the filter was less than $(<) 1$ drop per min. Filters (or filter series) containing genomic DNA were preserved in ethanol and refrigerated at $4{ }^{\circ} \mathrm{C}$ until they were shipped overnight to the USGS San Diego Field Station for DNA extraction. We washed the filter funnel with 50 percent bleach solution, rinsed the filter funnel and clamps with deionized water, and allowed them to air dry between samples.

We used the PowerWater ${ }^{\circledR}$ DNA isolation kit (MoBio Laboratories Inc., Carlsbad, California) with the vacuum manifold protocol option as our primary method of eDNA extraction (fig. 5). We eluted the final volume of DNA in $50 \mu \mathrm{L}$ of elution buffer that was supplied with each kit. We prepared and processed all field negatives in the same way.

We also assessed efficiency of eDNA capture and extraction on a subset $(n=6)$ of fieldcollected water samples using Sterivex ${ }^{\mathrm{TM}}$-GP polyethersulfone filter capsules (EMD Millipore, Darmstadt, Germany) and the PowerWater ${ }^{\circledR}$ Sterivex ${ }^{\mathrm{TM}}$ DNA Isolation kit (MoBio Laboratories Inc., Carlsbad, California; fig. 5) because recent studies have found these filters to yield improved capture efficiency (Bergman and others, 2016, Spens and others, 2017). These samples were collected at some of the same sites as all other samples, but during October 2016. For these samples, we filtered water directly from the source at field sites until water no longer passed through the filter. We recorded the volume of water filtered at each sampling location; sampled volumes had a mean of $348 \mathrm{~mL}$ (range, 110-800 mL). Sterivex ${ }^{\mathrm{TM}}$ filters containing genomic DNA were refrigerated at $4{ }^{\circ} \mathrm{C}$ and shipped overnight to the USGS San Diego Field Station for DNA extraction. We made a modification at step 17 of the supplied extraction protocol by reducing the vortex time to $3 \mathrm{~min}$ (instead of $5 \mathrm{~min}$ ) to reduce the amount of DNA shearing, and eluted the final volume of DNA in $50 \mu \mathrm{L}$ of elution buffer that was supplied with the kit.

\section{Precipitation Techniques}

Although most eDNA studies use a filtration method to capture eDNA, some studies have effectively used precipitation as a means to capture eDNA (Piaggio and others, 2014; Deiner and others, 2015). Therefore, we evaluated whether eDNA capture from precipitation would produce more efficient recovery and concentration of giant gartersnake eDNA on a subset of water samples (fig. 5). To do this, we used the additional 1-L water samples that were collected during the October 1, 2015, sampling occasion. Water samples were preserved with the same procedures as our filtered water samples (addition of $1 \mathrm{~mL}$ of $3 \mathrm{M}$ sodium acetate and $33 \mathrm{~mL}$ of 95 percent ethanol) and were stored at $-20{ }^{\circ} \mathrm{C}$. Prior to extractions, we thawed each water sample overnight in a $4{ }^{\circ} \mathrm{C}$ refrigerator. Once thawed, samples were placed on a magnetic stir plate for $10 \mathrm{~min}$ to thoroughly mix the water column. Once mixed, three $50 \mathrm{~mL}$ subsamples were removed, placed in $50 \mathrm{~mL}$ conical centrifuge tubes, and immediately centrifuged $(3,220 \mathrm{~g}, 45 \mathrm{~min})$ to form a precipitation pellet of the suspended debris. We decanted off the excess water, inverted the tubes on paper towels to dry, and performed eDNA extraction on the recovered pellet at the bottom of the conical tube. We tested two DNA extraction kits 
that have been used in other studies using precipitation extraction techniques (Piaggio and others, 2014, Williams and others, 2017): DNeasy ${ }^{\circledR}$ mericon ${ }^{\circledR}$ Food kit (Qiagen, Inc., Valencia, California) and the QIAamp ${ }^{\circledR}$ DNA mini kit (Qiagen, Inc., Valencia, California). For the DNeasy ${ }^{\circledR}$ mericon ${ }^{\circledR}$ Food kit, we followed the standard protocol for a $200-\mathrm{mg}$ sample, with the exception of eluting in a final of $80 \mu \mathrm{L}$ of

elution buffer. For the QIAamp ${ }^{\circledR}$ DNA mini kit, we resuspended the pellet after centrifugation in $300 \mu \mathrm{L}$ of Qiagen ATL buffer before following the standard extraction protocol. All filtered and precipitation DNA extractions were quantified using a Qubit ${ }^{\circledR} 2.0$ Fluorometer and stored at $-20{ }^{\circ} \mathrm{C}$ until used for quantitative PCR analyses.

\section{Spike Test}

Because positive detections from laboratory and field-collected samples were rare (see section "Results"), we performed a spiked water test to determine the efficiency of our preferred filtration and extraction workflow (fig. 5). Water was collected from a field site at which extensive trapping has occurred, but giant gartersnakes were never detected visually or in traps; $1 \mathrm{~L}$ of water was placed into each of eight plastic containers. Two additional plastic containers were each filled with $1 \mathrm{~L}$ of molecular-grade water as controls for analysis of PCR inhibitors. DNA extracted from a giant gartersnake tail-tip $(36.4 \mathrm{ng} / \mu \mathrm{L})$ was spiked into each container- $-1 \mu \mathrm{L}$ into four field water samples and $10 \mu \mathrm{L}$ into four field water samples. We also spiked the two control containers filled with molecular grade water with 1 and $10 \mu \mathrm{L}$ of giant gartersnake DNA. Water samples were processed using the 0.45 $\mu \mathrm{m}$ nitrocellulose filters and extracted with the PowerWater ${ }^{\circledR}$ DNA isolation kit (Qiagen, Inc., Valencia, California). Each set ( 1 and $10 \mu \mathrm{L})$ of samples was processed with several assay trials outlined in the next section (that is, standard quantitative PCR [qPCR], pre-qPCR, and PCR inhibitor removal kits).

\section{PCR and qPCR Assay Development}

\section{Species-Specific Marker Design}

To obtain multi-species sequence alignments, we extracted gDNA from tissue samples (muscle and tail-tips) of giant gartersnakes and closely related and (or) potentially sympatric species (common gartersnake, T. sirtalis; terrestrial gartersnake, T. elegans; aquatic gartersnake, T. atratus; sierra gartersnake, T. couchii; and banded watersnake, Nerodia fasciata) throughout or near the Central Valley acquired from a previous study (Wood and others, 2015), our own field collections, and museum loans. We used the Qiagen DNeasy ${ }^{\circledR}$ Blood and Tissue Kit (Qiagen, Inc., Valencia, California) to isolate gDNA and assessed the quality and quantity of recovered DNA using a Qubit ${ }^{\circledR} 2.0$ Fluorometer.

Primer and probe designs were developed using multi-species alignments from the mitochondrial DNA (mtDNA) sequence data from our DNA samples and those downloaded from GenBank (Vector NTI ${ }^{\mathrm{TM}}$; InforMax Inc., North Bethesda, Maryland). Alignments consisted of the giant gartersnake, banded watersnake, and the four closely related and (or) potentially sympatric Thamnophis species listed in the preceding paragraph to identify highly differentiated species-specific regions in the nicotinamide adenine dinucleotide (reduced form) (NADH) dehydrogenase subunit 4 (ND4), NADH dehydrogenase subunit 2 (ND2), and Cytochrome b (Cytb) genes (appendixes 1-4). Candidate primer sets for the three genes were screened for positive amplification using conventional PCR techniques 
following the mastermix and cycle requirements recommended for TaqMan ${ }^{\circledR}$ Universal Master Mix II (Applied Biosystems, Foster City, California) on 1-10 g DNA samples for each species within T. gigas and $N$. fasciata. The PCR products that were positive for the two target species (T. gigas and $N$. fasciata) were purified using the Qiagen PCR Purification kit for PCR clean-up (Germantown, Maryland). We confirmed that amplified products were the desired target species using Sanger sequencing on an Applied Biosystems, Inc. (ABI) 3730 Capillary Electrophoresis Genetic Analyzer (University of California, Davis, DNA Sequencing Laboratory).

\section{Quantitative PCR}

In order to obtain high levels of primer sensitivity and specificity of DNA from water samples, we further modified sets of primers for use in qPCR TaqMan ${ }^{\circledR}$ assays with the addition of a probe (table 1). The probes were fluorescently labeled with 6-Carboxyflourescein (6-FAM) and 3' Iowa Black ${ }^{\circledR} \mathrm{FQ}$ probe quencher (IBFQ) (5' and 3', respectively). Following Applied Biosystems TaqMan ${ }^{\circledR}$ protocols, the theoretical lower limit of final probe concentration was determined to allow for yields of the minimum quantity for each target. Probe dilution concentration was 250 nanomolar (nM) in $10-n M$ increments. All PCR and qPCR set up was conducted in a separate lab facility (Davis Field Station) than filtration and precipitation processing, and no gartersnake tissue samples were ever handled in this facility. We developed two qPCR assays for evaluation: (1) a standard qPCR assay; and (2) a pre-qPCR assay. For our standard qPCR assay, each $20-\mu \mathrm{L}$ reaction contained $12.5 \mu \mathrm{L}$ of $1 \mathrm{X}$ TaqMan ${ }^{\circledR}$ Universal Master Mix II (Applied Biosystems, Foster City, California), $0.5 \mu \mathrm{L}$ of 250-nM Integrated DNA Technologies dual-labeled DNA probe (IDT_LNA), $1 \mu \mathrm{L}$ of $500-\mathrm{nM}$ of each IDT primer, and $5 \mu \mathrm{L}$ of gDNA (IDT, Coralville, Iowa). An ABI StepOne-Plus Real-Time PCR system was used with the Fast qPCR profile consisting of $50{ }^{\circ} \mathrm{C}(2 \mathrm{~min}), 95^{\circ} \mathrm{C}(10 \mathrm{~min})$, followed by 40 cycles of $95{ }^{\circ} \mathrm{C}(15 \mathrm{sec})$ and $60{ }^{\circ} \mathrm{C}(1 \mathrm{~min})$ annealing temperatures. For our pre-qPCR assay, samples were subjected to a PCR amplification with the unlabeled IDT primers prior to running the standard qPCR assay with the fluorescently labeled primers and probe. We used the Clontech (Takara Bio USA, Inc.) Advantage ${ }^{\circledR} 2$ PCR protocol to amplify samples, and each $50-\mu \mathrm{L}$ reaction contained $36 \mu \mathrm{L}$ of PCR-Grade Water, $5 \mu \mathrm{L}$ of 10X Advantage ${ }^{\circledR} 2$ Short Amplicon (SA) PCR Buffer, $1 \mu \mathrm{L}$ of $500 \mathrm{nM}$ of each IDT unlabeled primer (Integrated DNA Technologies [IDT], Coralville, Iowa), $1 \mu \mathrm{L}$ of 50X Advantage ${ }^{\circledR} 2$ Polymerase Mix and $5 \mu \mathrm{L}$ of gDNA. The PCR was performed on an MJ Research Inc. (MJ) PTC-200 thermal cycler (MJ, Watertown, Massachusetts) and consisted of one cycle at $95{ }^{\circ} \mathrm{C}$ for $1 \mathrm{~min}$, and then 20 cycles at 95 ${ }^{\circ} \mathrm{C}$ for $30 \mathrm{sec}$, at $60{ }^{\circ} \mathrm{C}$ for $1 \mathrm{~min}$, and $68^{\circ} \mathrm{C}$ for $30 \mathrm{sec}$, with a final extension step of $68^{\circ} \mathrm{C}$ for $1 \mathrm{~min}$. All pre-qPCR samples were then purified using the Qiagen QIAquick PCR Purification kit (Qiagen, Inc., Valencia, California), following the kit-specific protocol and then screened using the standard qPCR assay. For each set of qPCR primer/probes, we again tested one individual of the target species to confirm amplification, in addition to one individual of each of the other closely related species to confirm desired species specificity. 
Table 1. Primer and probe sets for dehydrogenase subunits 4 and 2 , and Cytochrome $b$ gene regions for giant gartersnake (Thamnophis gigas) and banded watersnake (Nerodia fasciata).

[We tested primer sets for dehydrogenase subunits 4 and 2 (ND4 and ND2), and provide additional primer sequences for Cytochrome b (Cytb). Probes were fluorescently labeled with 6-Carboxyflourescein ( 6-FAM) and 3' Iowa Black ${ }^{\mathbb{B}}$ FQ probe quencher (IBFQ). $\geq$, greater than or equal to]

\begin{tabular}{|c|c|c|c|c|}
\hline Species & $\begin{array}{c}\text { Gene } \\
\text { region }\end{array}$ & $\begin{array}{l}\text { Amplicon } \\
\text { length }\end{array}$ & Primer name & Primer sequence $\left(5^{\prime} \geq 3^{\prime}\right)$ \\
\hline \multirow[t]{9}{*}{ T. gigas } & ND4 & 120 & ND4_Forward & CAG-CAA-TCC-TTT-TAA-AAC-TAG-GA \\
\hline & & & ND4_Reverse & GCC-AGT-GTT-GCT-CCT-CAG \\
\hline & & & ND4_LNA Probe & 6FAM-TAA-TAC-AAA-CCC-TCC \\
\hline & ND2 & 141 & ND2_Forward & CAT-CAC-CGC-ATC-AAC-TAC-AAT \\
\hline & & & ND2_Reverse & TAG-GAT-GAG-TCA-TTT-AGG-TAT-AA \\
\hline & & & ND2_LNA Probe & 6FAM-ССТ-ТАТ-АCТ-АСТ-ТCT-ТTC-TAC-АAC-TA \\
\hline & Cytb & 134 & Cytb_Forward & GCC-TCA-CCA-CCT-GAC-TTT-GA \\
\hline & & & Cytb_Reverse & CTT-CGG-TGT-GTA-GTA-GCA-TG \\
\hline & & & Cytb_Probe & CCT-CCA-CTT-TAT-CCT-ACC-ATT \\
\hline \multirow[t]{6}{*}{ N. fasciata } & ND4 & 89 & ND4_Forward & TTC-TAT-CAA-CAC-AAA-TAA-ACC-CC \\
\hline & & & ND4_Reverse & GGG-TGT-GGA-GTA-GTA-TAA-GTA-G \\
\hline & & & ND4_LNA Probe & 6FAM-TTC-AAC-CTT-CAC-AC \\
\hline & $\mathrm{ND} 2$ & 137 & ND2_Forward & AGC-ATT-AAC-CTC-ACT-ACT-CAG \\
\hline & & & ND2_Reverse & GTT-GAG-GCC-ACT-ATT-GTG-TG \\
\hline & & & ND2_LNA Probe & 6FAM-ССТ-ТАТ-АCТ-АСТ-ТCТ-ТTC-TAC-АAC-TA \\
\hline
\end{tabular}

\section{Capture-Mark-Recapture Sampling}

We conducted capture-mark-recapture studies at each of the eDNA water sampling sites during 2015. Because the canal and wetland habitat sites at the BKS tract were near each other, we treated them as a single population and trapped them with 150 traps during April 15, 2015-July 2, 2015 (fig. 1). At Colusa National Wildlife Refuge (NWR), the canal and wetland sites were farther apart, and we trapped these sites separately (figs. 2 and 3). We trapped the Glenn-Colusa Irrigation District (GCID) canal with 50 traps during June 17, 2015-September 20, 2015. We trapped East Pond with 50 traps beginning on June 18, 2015, but low water levels caused by drought forced us to remove the traps on June 22, 2015. 
Therefore, we also report trapping results from 2014 (50 traps during July 18-August 31) and 2016 (50 traps during August 23-September 16) to provide evidence for the occurrence and abundance of snakes at this site. At all sites, we modified commercially available aquatic funnel traps (Tackle Factory, Fillmore, New York) so that they would float, to extend the large opening of the funnel to increase the likelihood of individuals being directed to the trap opening, and by placing cable ties along the small opening of the funnel to prevent the escape of captured individuals (Casazza and others, 2000; Halstead and others, 2013). We positioned transects along the banks or at the edges of emergent vegetation in wetlands, or along the edges of canals, which all act as natural drift fences that direct snake movement to traps. We monitored environmental conditions relevant to giant gartersnake behavior daily at each transect. In particular, we measured water temperatures, air temperatures, sky condition (cloud cover or haze), and fluctuations in water level. We measured, sexed, and uniquely marked each captured giant gartersnake, and released it at its capture location immediately after processing (Wylie and others, 2010).

\section{Data Analysis}

\section{Quantitative PCR}

We analyzed all qPCR samples from laboratory and field trials in triplicate (technical replicates) and calculated the mean quantification cycle $\left(\mathrm{C}_{\mathrm{q}}\right)$ value from the three replicates. As qPCR is based on accumulation of a fluorescent signal during amplification, $\mathrm{C}_{\mathrm{q}}$ values are inverse to the amount of nucleic acid in each sample. Thus, a lower $\mathrm{C}_{\mathrm{q}}$ value corresponds to a greater amount of DNA. For both standard qPCR and pre-qPCR assays, we included negative controls (sterile water) and positive controls for the standard curve (DNA isolated from tissue samples) in each qPCR. We also included an additional negative control for the pre-qPCR assay that was included during the PCR amplification step prior to qPCR. To identify theoretical thresholds of qPCR detection, a 1:10 dilution of DNA was conducted from $10 \mu \mathrm{L}$ of gDNA with $90 \mu \mathrm{L}$ water for both target species and non-target species; $1 \mu \mathrm{L}$ of this dilution was pipetted into the wells. We developed relative standard curves (RSCs) from 1:10 serial dilutions of quantified target-species DNA as follows:

- For the giant gartersnake, $36.4 \mathrm{ng} / \mu \mathrm{L}, 3.64 \mathrm{ng} / \mu \mathrm{L}, 3.64 \times 10^{-1} \mathrm{ng} / \mu \mathrm{L}, 3.64 \times 10^{-2} \mathrm{ng} / \mu \mathrm{L}, 3.64 \times$ $10^{-3} \mathrm{ng} / \mu \mathrm{L} ;$ and

- For the banded watersnake, $23.2 \mathrm{ng} / \mu \mathrm{L}, 2.32 \mathrm{ng} / \mu \mathrm{L}, 2.32 \times 10^{-1} \mathrm{ng} / \mu \mathrm{L}, 2.32 \times 10^{-2} \mathrm{ng} / \mu \mathrm{L}, 2.32 \times$ $\left.10^{-3} \mathrm{ng} / \mu \mathrm{L}\right)$.

The identified RSC slope and y-intercept were used to calculate the DNA concentration for the measured $\mathrm{C}_{\mathrm{q}}$ values.

\section{Capture-Mark-Recapture}

We estimated the abundance of giant gartersnakes at each sampled location using Bayesian analysis of capture-mark-recapture (CMR) models using data augmentation (Royle and Dorazio, 2008). Data augmentation is an approach to CMR analysis in which a large number of all-zero capture histories of individuals that potentially were members of the population, but were unobserved, is appended to the observed capture histories. The abundance estimation problem then becomes analogous to the estimation of occupancy by seeking to answer the question, "how many undetected individuals were actually a part of the population but not observed?" This approach is much more flexible than other approaches to estimation of abundance and allows a unified framework for analysis of detectionnondetection and CMR data (Royle and Dorazio, 2008). 
We fit a full model containing effects of the following on daily individual capture probabilities:

- Sex,

- Snout-vent length (SVL),

- Water temperature,

- Date,

- An ephemeral behavioral response to capture (capture on day $t-1$ affected capture on day $t$, but effects did not persist), and

- Unexplained random temporal variation.

The model did not contain any interactions among variables. We standardized all continuous variables to improve behavior of the Markov chain Monte Carlo (MCMC) algorithm. East Pond was only set for 156 trap days because of water restrictions resulting in complete drying of the pond, and only one snake was captured in 2015. Because of the short sampling time, it was not included in abundance estimates, and we report abundance estimates and number of captures for 2014 and 2016, respectively, to indicate that giant gartersnakes likely occurred at the site continuously. For all abundance sites, we augmented the capture histories of trapped individuals with 500 all-zero capture histories. Five hundred pseudoindividuals was the number deemed adequate because the posterior density for abundance was less than 500 . We used uninformative priors for all parameters of all models-Beta $(1,1)$ for probabilities, $N(0,1.648)$ for regression coefficients, $U(0,10)$ for standard deviations, and $\operatorname{Bin}(1,0.5)$ for indicator variables.

Each model was run on three independent chains of 10,000 iterations each after a burn-in of 5,000 iterations; each chain was thinned by a factor of three. We analyzed each model by calling Just Another Gibbs Sampler (JAGS) 3.4.0 (Plummer, 2014a) from R 3.1.2 (R Core Team, 2014) using the R package rjags (Plummer, 2014b). We summarized posterior distributions with the posterior median and 95 percent credible interval (2.5 and 97.5 percent quantiles of the posterior distribution).

\section{Results}

\section{Species-Specific qPCR Marker Design}

We designed primers and probes for the giant gartersnake and banded watersnake using the mitochondrial ND4, ND2, and Cytb gene regions (table 1) and tested them for cross-species amplification for the five species of snakes used in the multi-species alignments. The qPCR primer/probe sets evaluated for ND2 consistently cross amplified the other Thamnophis species, so we did not explore their utility any further. For the giant gartersnake ND4 primer/probe set, we confirmed the absence of cross-species amplification across the other five species of snakes during qPCR and decided to use this set for further evaluation with laboratory and field eDNA samples. For the banded watersnake, we confirmed the absence of cross-species amplification for the ND4 primer/probe set, but did not evaluate their utility to detect this species with eDNA any further. Similarly, we developed primer and probe sets for Cytb for the giant gartersnake, but we did not evaluate their utility for eDNA applications given the confirmed absence of cross-species amplification using the ND4 primer/probe set. We used serial dilutions of known amounts of gDNA to determine the theoretical lower limits of detection for the giant gartersnake and banded watersnake using these primer/probe sets. The giant gartersnake had a RSC threshold at $3.64 \times 10^{-3} 3 \mathrm{ng} / \mu \mathrm{L}\left(\mathrm{C}_{\mathrm{q}}=36\right)$, which we used as the standard cutoff value for presence/absence (that is, $\mathrm{C}_{\mathrm{q}}<36$ indicates detection). We used the standard curves to estimate copy numbers of unknown samples. 


\section{Laboratory Experiment}

eDNA was extracted from the 34 laboratory water samples having undergone four different treatments (that is, deionized [DI] water tank control, shed skin, live snake, and snake feces). Filtration of 1-L volume of water from each tank was performed as described above (Methods: Laboratory Experiment) except in three instances where multiple filters were used to completely filter the 1-L water sample. In these cases, we extracted the filters separately and combined the supernatant during the column binding step of the PowerWater ${ }^{\circledR}$ DNA isolation kit (Step 16). DNA concentrations from the negative control and DI water control tanks were all less than $0.05 \mathrm{ng} / \mu \mathrm{L}$. DNA concentrations from the treatments ranged from $<0.05$ to $0.225 \mathrm{ng} / \mu \mathrm{L}$ (live snake), $<0.05$ to $2.03 \mathrm{ng} / \mu \mathrm{L}$ (shed skin), and $<0.05$ to $3.2 \mathrm{ng} / \mu \mathrm{L}$ (snake feces). For the standard qPCR assay, we recovered $\mathrm{C}_{\mathrm{q}}$ values that were beyond the limits of detection for all samples and treatments. For the pre-qPCR assay, we recovered $\mathrm{C}_{\mathrm{q}}$ values that were beyond the limits of detection for the live snake and control treatments. However, we recovered $\mathrm{C}_{\mathrm{q}}$ values corresponding to positive detection for the shed skin and snake feces treatments using the preqPCR assay (table 2). For the shed skin treatment, positive detection occurred after $21 \mathrm{~d}$ (shed skin $\mathrm{C}_{\mathrm{q}}$ value 15 or $657.97 \mathrm{ng} / \mu \mathrm{L})$, but not for any previous water samples $(1-16 \mathrm{~d})$. For the snake feces, positive detection occurred after 1,16 , and $21 \mathrm{~d}\left(\mathrm{C}_{\mathrm{q}}\right.$ values 21,23 , and 29$)$.

Table 2. Detection results using the pre-quantitative polymerase chain reaction assay for giant gartersnakes from the different treatments (that is, deoxyribonucleic acid source) from the laboratory trials.

[All samples were run in triplicate; average quantification cycle $\left(\mathrm{C}_{\mathrm{q}}\right)$ values across the three replicates are reported in bold for positive detections. If $\mathrm{C}_{\mathrm{q}}$ values exceeded the detection threshold $\left(\mathrm{C}_{\mathrm{q}}\right.$ values $\left.>36\right)$, they were scored as negative for detection. Note that $\mathrm{C}_{\mathrm{q}}$ values $<36$ indicate positive detections]

\begin{tabular}{lccccccc}
\hline Treatment & $\mathbf{0}$ day & $\mathbf{1}$ day & $\mathbf{2}$ days & $\mathbf{3}$ days & $\mathbf{8}$ days & $\mathbf{1 6}$ days & $\mathbf{2 1}$ days \\
\hline Control tank & Negative & Negative & Negative & Negative & Negative & Negative & Negative \\
Shed skin & Negative & Negative & Negative & Negative & Negative & Negative & $\begin{array}{c}\text { Positive } \\
\left(\mathbf{C}_{\mathbf{q}}: \mathbf{1 5}\right)\end{array}$ \\
Live snake & Negative & Negative & Negative & Negative & Negative & Negative & Negative \\
Snake feces & Negative & $\begin{array}{c}\text { Positive } \\
\left(\mathbf{C}_{\mathbf{q}}: \mathbf{2 1}\right)\end{array}$ & Negative & Negative & Negative & $\begin{array}{c}\text { Positive } \\
\left(\mathbf{C}_{\mathbf{q}}: \mathbf{2 3}\right)\end{array}$ & $\begin{array}{c}\text { Positive } \\
\left(\mathbf{C}_{\mathbf{q}}: \mathbf{2 9}\right)\end{array}$ \\
\hline
\end{tabular}




\section{Field Experiment}

Across all capture methods, DNA concentrations were sufficient to detect giant gartersnakes, assuming that giant gartersnake DNA was present in the eDNA samples. The concentration of total DNA from our field-collected water ranged from $<0.05$ to $29.1 \mathrm{ng} / \mu \mathrm{L}$ for filtered capture methods using the PowerWater ${ }^{\circledR}$ DNA Isolation kit and PowerWater ${ }^{\circledR}$ Sterivex ${ }^{\mathrm{TM}}$ DNA Isolation kit, and from 0.27 to $9.47 \mathrm{ng} / \mu \mathrm{L}$ for the precipitation capture method using the DNeasy ${ }^{\circledR}$ mericon ${ }^{\circledR}$ Food and QIAamp ${ }^{\circledR}$ DNA mini kits.

Quantitative PCR assay controls provided expected positive and negative results for each assay. However, all field trial assays were negative for presence of giant gartersnakes and (or) banded watersnakes using the standard qPCR and pre-qPCR assays (table 3).

Although we did not detect giant gartersnakes with eDNA at the field locations, they were detected with traps at all sites. We captured 35 individual giant gartersnakes a total of 41 times at the combined canal and wetland sites at BKS, and we estimated model-averaged abundance to be 250 (95 percent CI, 94-506) giant gartersnakes. At East Pond in Colusa NWR, we captured one giant gartersnake in 2015 and 11 giant gartersnakes in 2016 (each individual was captured once). At East Pond in 2014, we captured 30 individual giant gartersnakes a total of 46 times, and we estimated modelaveraged abundance to be 115 (95 percent CI, 43-468) giant gartersnakes. At the GCID canal, we captured 34 individual giant gartersnakes 60 times, and we estimated model-averaged abundance to be 53 (95 percent CI, 39-133) giant gartersnakes. We did not detect any banded watersnakes in traps.

Table 3. Quantitative polymerase chain reaction (qPCR) results from field-collected water samples by habitat type and sample period using pre-qPCR assay.

[All samples were run in triplicate; quantification cycle $\left(\mathrm{C}_{\mathrm{q}}\right)$ values across the three replicates were averaged. If $\mathrm{C}_{\mathrm{q}}$ values exceeded the detection threshold $\left(\mathrm{C}_{\mathrm{q}}\right.$ values $\left.>36\right)$, they were scored as negative for detection. DNA, deoxyribonucleic acid; $\mathrm{N}$, number of samples; \# rxns, number of reactions]

\begin{tabular}{llcll}
\hline Habitat type & Sample period & $\begin{array}{c}\text { N } \\
\text { (\# rxns) }\end{array}$ & $\begin{array}{c}\text { Average total DNA } \\
\text { concentration (ng/uL) }\end{array}$ & Detection \\
\hline Wetland & March & $11(33)$ & $0.86(0.05-4.30)$ & Negative \\
Wetland & July & $6(18)$ & $2.6(0.21-8.01)$ & Negative \\
Wetland & October & $6(18)$ & $4.5(0.49-14.5)$ & Negative \\
Wetland total & & $\mathbf{2 3 ( 6 9 )}$ & & \\
Canal & March & $15(45)$ & $0.37(0.05-2.00)$ & Negative \\
Canal & July & $6(18)$ & $3.08(0.92-5.91)$ & Negative \\
Canal & October & $6(18)$ & $2.43(0.05-10.30)$ & Negative \\
Canal total & & $\mathbf{2 7}(\mathbf{8 1})$ & & \\
\hline
\end{tabular}




\section{Spike Tests}

Spiked DNA was not detected for most of the tests attempted (table 4). The only detectable results were from the samples that were subjected to the pre-qPCR assay; $1-\mu \mathrm{L}$ spike tests with preqPCR resulted in average $C_{q}$ values of 19.0 , whereas $10-\mu \mathrm{L}$ spike tests resulted in average $C_{q}$ values of 15.6 (table 5). Control samples spiked with $10 \mu \mathrm{L}$ of DNA, having been extracted and processed without any optimization techniques, were at the margin of detection using quantitative PCR with average $C_{q}$ values of 36.8. Samples that were treated with PCR inhibitor removal kits (GeneReleaser ${ }^{\circledR}$ and OneStep ${ }^{\mathrm{TM}}$ ) prior to qPCR assays did not improve the efficiency of capture and detection. Efficiency of extraction was calculated using the pre-qPCR assay samples and the equation of [(nanograms DNA recovered, as clarified by the $\mathrm{C}_{\mathrm{q}}$ value relationship to the standard curve)/nanograms DNA spiked $) \times 100]$. Efficiency of recovery at $1-\mu \mathrm{L}$ spike was 55 percent $[(20.2 \mathrm{ng}$ recovered/36.4 $\mathrm{ng}$ spiked $) \times 100]$ and efficiency at $10-\mu \mathrm{L}$ spike was 38 percent $[(140 \mathrm{ng}$ recovered/364 ng spiked $) \times 100]$.

Table 4. Quantitative polymerase chain reaction (qPCR) results from precipitation capture and extraction techniques run with standard and pre-qPCR assays.

[The two environmental deoxyribonucleic acid extraction kits used were QIAamp ${ }^{\circledR}$ DNA mini kit (Qiagen, Inc.) and DNeasy ${ }^{\circledR}$ Mericon ${ }^{\circledR}$ Food kit (Qiagen, Inc.). $\mathrm{C}_{\mathrm{q}}$, quantification cycle; N, number of samples; rxns, number of reactions; >, greater than]

\begin{tabular}{lccc} 
Assay type & $\begin{array}{c}\text { QIAamp } \\
\mathbf{N}(\mathbf{r x n s})\end{array}$ & $\begin{array}{c}\text { DNeasy }{ }^{\circledR} \text { mericon } \\
\mathbf{N}(\mathbf{r x n s})\end{array}$ & Detection \\
\hline Standard qPCR & $5(15)$ & $4(12)$ & Negative $\left(\mathrm{C}_{\mathrm{q}}>36\right)$ \\
pre-qPCR & $5(15)$ & $4(12)$ & Negative $\left(\mathrm{C}_{\mathrm{q}}>36\right)$ \\
\hline
\end{tabular}

Table 5. Spiked deoxyribonucleic acid test results to evaluate the efficiency of filtration and capture techniques.

[Water was collected at Tule Basin field site, a site where giant gartersnakes have not been detected, despite extensive trapping effort. All samples were run in triplicate; average quantification cycle $\left(\mathrm{C}_{\mathrm{q}}\right)$ values across the three replicates are reported in bold for positive detections. If $\mathrm{C}_{\mathrm{q}}$ values exceeded the detection threshold $\left(\mathrm{C}_{\mathrm{q}}\right.$ values $\left.>36\right)$, they were scored as negative for detection. DNA, deoxyribonucleic acid; N, number of samples; \# rxns, number of reactions; PCR, polymerase chain reaction; qPCR, quantitative PCR; uL, microliter]

\begin{tabular}{llcllll}
\hline $\begin{array}{c}\text { Water replicates } \\
\text { (1 liter) }\end{array}$ & $\begin{array}{c}\text { Amount of DNA } \\
\text { added } \\
\text { (uL) }\end{array}$ & $\begin{array}{c}\mathbf{N} \\
\text { (\# rxns) }\end{array}$ & $\begin{array}{c}\text { Standard } \\
\text { qPCR }\end{array}$ & pre-qPCR & $\begin{array}{c}\text { OneStep }{ }^{\mathrm{TM}} \\
\text { inhibitor- } \\
\text { pre-PCR }\end{array}$ & $\begin{array}{c}\text { GeneReleaser } \\
\text { inhibitor-pre- } \\
\text { PCR }\end{array}$ \\
\hline Field samples & 1 -uL spike & $4(48)$ & Negative & $\begin{array}{c}\text { Positive }\left(\mathbf{C}_{\mathbf{q}}:\right. \\
\mathbf{1 9 . 0})\end{array}$ & Negative & Negative \\
& 10 -uL spike & $4(48)$ & Negative & $\begin{array}{c}\text { Positive }\left(\mathbf{C}_{\mathbf{q}}:\right. \\
\mathbf{1 5 . 6})\end{array}$ & Negative & Negative \\
Control samples & 1 -uL spike & $2(24)$ & Negative & Negative & Negative & Negative \\
& $10-\mathrm{uL}$ spike & $2(24)$ & Negative & Negative & Negative & Negative \\
\hline
\end{tabular}




\section{Discussion}

Even under ideal laboratory conditions, detection of giant gartersnake eDNA in water was not always successful. Unlike amphibian and fish skin, reptile skin is highly keratinized with $\beta$-keratin and impermeable to water, leaving the mouth and cloaca as the only potential surfaces to shed DNA into the water column. The impermeability of reptile skin to water was further evidenced by the pattern of detection of eDNA in the shed skin treatment; only after soaking for $21 \mathrm{~d}$ was eDNA detectable in the shed skin treatment. The fecal treatment had the highest detectability, but was still only detectable in three of the six samples after being introduced into the water. The pattern of detection (after 1, 16, and $21 \mathrm{~d}$, with nondetection at 1,3, and $8 \mathrm{~d}$ ) indicates that the concentration of giant gartersnake DNA left behind in feces was too small to be reliably detected in a 1-L sample of water in this treatment.

The low detectability of giant gartersnake eDNA in the laboratory was not improved by field conditions. Despite relatively high abundances of giant gartersnakes at all sites, we failed to detect giant gartersnake DNA in any field samples. Several aspects of giant gartersnake biology likely contribute to the limitations of detecting this species with eDNA. As mentioned in the preceding paragraph, the $\beta$ keratin in reptile skin is impermeable to water, and limits the amount of DNA shed by snakes while they are in water. Further exacerbating the limited DNA shed by reptiles is that the average adult female giant gartersnake spends 59 (95 percent CI, 53-66) percent of its time on land (albeit very close to water) in mid-July - the amount of time spent on land is greater earlier and later in the active season, and reaches 100 percent during brumation (Halstead and others, 2015). Large males make even more extensive use of the terrestrial environment (68 [95 percent CI, 57-77] percent; Halstead and others, 2015), and it is unknown to what extent juvenile and smaller adult snakes use the aquatic environment. If giant gartersnakes also primarily defecate and shed on land, the opportunity to detect their DNA in water could be extremely limited.

In addition to species characteristics, the physical and chemical characteristics of the environment likely further limit detection of giant gartersnakes by eDNA. The Mediterranean climate of the California Central Valley, combined with the marsh habitat of giant gartersnakes, exposes DNA near the top of the water column to high temperatures and intense ultraviolet (UV) radiation, which may quickly degrade the limited amount of giant gartersnake DNA in the water. Stickler and others (2015) showed that eDNA degradation rates were lowest in aquatic habitats with cooler temperatures $\left(5^{\circ} \mathrm{C}\right)$ and protection from solar radiation (that is, low UV radiation levels). Additionally, the water in canals and wetlands often contains large amounts of suspended solids, especially fine clay particles that could bind to DNA and make it less available for extraction. Many agricultural chemicals, including herbicides, pesticides, and fertilizers, are heavily applied in the Central Valley; it is unknown how this chemical environment might also affect the persistence or extraction of DNA.

Based on our results, eDNA is not currently a viable alternative to trapping to determine the occurrence of giant gartersnakes. For comparison, using a simple binomial model for detection probability at the level of effort for field sampling at each of the sites, daily detection probability for BKS was 0.36 (95 percent credible interval [CI], 0.26-0.47), for East Pond was 0.26 (95 percent CI, 0.03-0.67), and for GCID was 0.85 (95 percent CI, 0.76-0.91). In $4 \mathrm{~d}$ of sampling (chosen to represent setting traps on Monday and removing them on Friday), the cumulative detection probability at each of these sites would be 0.84 (95 percent CI, 0.71-0.93), 0.90 (95 percent CI, 0.27-greater than 0.99), and $>0.99$ (95 percent CI, greater than 0.99-greater than 0.99) at BKS, East Pond, and GCID, respectively. Despite the good performance of traps for detecting giant gartersnakes at these and other sites where they are abundant, detecting giant gartersnakes with eDNA remains appealing. The ability to detect species simply by filtering water and extracting and amplifying DNA has many advantages both to researchers and the organisms, and we hope that future development of technology will enable detection 
of lower and lower concentrations of DNA to the point where detection of giant gartersnakes using this method is feasible. On the basis of this study, the preferred workflow used $1 \mathrm{~L}$ of water sample, captured eDNA through filters using a $0.45-\mu \mathrm{m}$ nitrocellulose filter, extracted eDNA using the PowerWater ${ }^{\circledR}$ DNA kit, and amplified target DNA with the pre-qPCR assay without the need for further processing with inhibitor removal kits. However, additional tests for inhibition and exploration of methods for inhibitor removal might be productive avenues for future assay development. As a supplement to this workflow, we suggest using larger amounts of water $(>1 \mathrm{~L})$ as a possible means to increase eDNA detection. Filtration methods have been used to process larger volumes as great as $5 \mathrm{~L}$ (Goldberg and others, 2016), but the suspended particulate matter (that is, organic and inorganic materials) in the water from the sites we sampled quickly clogged the filters and remained a challenge for processing even $1 \mathrm{~L}$ of water. Therefore, we also suggest evaluating whether using larger filter pore sizes (for example, $1-10 \mu \mathrm{m})$ — to accommodate the higher volume of water to be filtered $(\geq 5 \mathrm{~L})$ affects the concentration of giant gartersnake eDNA captured and increases detectability (Turner and others, 2014). Finally, we developed several sets of primer/probes using other gene regions that could be further evaluated for eDNA detection applications (appendixes 1-4). Until then, however, trapping remains the best method for evaluating the occurrence of this rare snake.

\section{Acknowledgments}

We thank Caren Goldberg (Washington State University) and Jonathan Richmond (USGS) for constructive reviews of an earlier version of this report. Lisa Parker (USGS) provided administrative support. The Natomas Basin Conservancy and the U.S. Fish and Wildlife Service Sacramento National Wildlife Refuge complex provided site access and logistical support. Snakes were handled in accordance with IACUC Protocol WERC-2014-01 and as stipulated in U.S. Fish and Wildlife Service Recovery Permit TE-020548-5 and California Department of Fish and Wildlife Scientific Collecting Permit 10779.

\section{References Cited}

Bergman, P.S., Schumer G., Blankenship S., and Campbell, E., 2016, Detection of adult green sturgeon using environmental DNA analysis: PLoS ONE, v. 11, p. e0153500.

California Fish and Game Commission, 1971, Animals of California declared to be endangered or threatened: California Code of Regulations, title 14, section 670.5.

Casazza, M.L., Wylie, G.D., and Gregory, C.J., 2000, A funnel trap modification for surface collection of aquatic amphibians and reptiles: Herpetological Review, v. 31, p. 91-92.

Deiner K., Walser J.-C., Mächler, E., and Altermatt, F., 2015, Choice of capture and extraction methods affect detection of freshwater biodiversity from environmental DNA: Biological Conservation, v. 183, p. 53-63.

DeJean, T., Valentini, A., Duparc, A., Pellier-Cuit, S., Pompanon, F., Taberlet, P., and Miaud, C., 2011, Persistence of environmental DNA in freshwater systems: PLoS One, v. 6, p. e23398.

Durso, A.M., Willson, J.D., and Winne, C.T., 2011, Needles in haystacks-Estimating detection probability and occupancy of rare and cryptic snakes: Biological Conservation, v. 144, p. 1,5081,515 .

Ficetola, G.F., Miaud, C., Pompanon, F., and Taberlet, P., 2008, Species detection using environmental DNA from water samples: Biology Letters, v. 4, p. 423-425.

Fitch, H.S., 1940, A biogeographical study of the ordinoides artenkreis of garter snakes (genus Thamnophis): Berkeley, University of California Publications in Zoology, v. 50, p. 485-530. 
Goldberg, C.S., Pilliod, D.S., Arkle, R.S., and Waits, L.P., 2011, Molecular detection of vertebrates in stream water-A demonstration using Rocky Mountain tailed frogs and Idaho giant salamanders: PLoS One, v. 6, p. e22746.

Goldberg, C.S., Strickler, K.M., and Pilliod, D.S., 2015, Moving environmental DNA methods from concept to practice for monitoring aquatic macroorganisms: Biological Conservation, v. 183, p. 1-3.

Goldberg, C.S., Turner, C.R., Deiner, K., Klymus, K.E., Thomsen, P.F., Murphy, M.A., Spear, S.F., McKee, A., Oyler-McCance, S.J., Cornman, R.S., Laramie, M.B., Mahon, A.R., Lance, R.F., Pilliod, D.S., Strickler, K.M., Waits, L.P., Fremier, A.K., Takahara, T., Herder, J.E., and Taberlet, P., 2016, Critical considerations for the application of environmental DNA methods to detect aquatic species: Methods in Ecology and Evolution, v. 7, p. 1,299-1,307.

Halstead, B.J., Skalos, S.M, Wylie, G.D., and Casazza, M.L., 2015, Terrestrial ecology of semi-aquatic giant gartersnakes (Thamnophis gigas): Herpetological Conservation and Biology, v. 10, p. 633-644.

Halstead, B.J., Wylie, G.D., and Casazza, M.L., 2010, Habitat suitability and conservation of the Giant Gartersnake (Thamnophis gigas) in the Sacramento Valley of California: Copeia, v. 2010, p. 591-599. Halstead, B.J., Wylie, G.D., and Casazza, M.L., 2013, Efficacy of trap modifications for increasing capture rates of aquatic snakes in floating aquatic funnel traps: Herpetological Conservation and Biology, v. 8, p. 65-74.

Halstead, B.J., Wylie, G.D, Casazza, M.L., and Coates, P.S., 2011, Bayesian adaptive survey protocols for resource management: The Journal of Wildlife Management, v. 75, p. 159-160.

Hunter, M.E., Oyler-McCance, S.J., Dorazio, R.M., Fike, J.A., Smith, B.J., Hunter, C.T., Reed, R.N., and Hart, K.M., 2015, Environmental DNA (eDNA) sampling improves occurrence and detection estimates of invasive Burmese pythons: PLoS One, v. 10, p. e0121655.

Jerde, C.L., Mahon, A.R., Chadderton, W.L., and Lodge, D.M., 2011, "Sight-unseen" detection of rare aquatic species using environmental DNA: Conservation Letters, v. 4, p. 150-157.

Kirshtein, J.D., Anderson, C.W., Wood, J.S., Longcore, J.E., and Voytek, M.A., 2007, Quantitative PCR detection of Batrachochytrium dendrobatidis DNA from sediments and water: Diseases of Aquatic Organisms, v. 77, p. 11-15.

McKee, A.M., Spear, S.F., and Pierson, T.W., 2015, The effect of dilution and the use of a postextraction nucleic acid purification column on the accuracy, precision, and inhibition of environmental DNA samples: Biological Conservation, v. 183, p. 70-76.

McKee, A.M., Calhoun, D.L., Barichivich, W.M., Spear, S.F., Goldberg, S.S, and Glenn, T.C., 2015, Assessment of environmental DNA for detecting presence of imperiled aquatic amphibian species in isolated wetlands: Journal of Fish and Wildlife Management, v. 6, p. 498-510.

Piaggio, A.J., Engeman, R.M., Hopken, M.W., Humphrey, J.S., Keacher, K.L., Bruce, W.E., and Avery, M.L., 2014, Detecting an elusive invasive species - A diagnostic PCR to detect Burmese python in Florida waters and an assessment of persistence of environmental DNA: Molecular Ecology Resources, v. 14, p. 374-380.

Plummer, M., 2014a, JAGS software version 3.4.0. https://sourceforge.net/projects/mcmc-jags/ (accessed 6 September 2017).

Plummer, M., 2014b, rjags_-Bayesian graphical models using MCMC, R package version 3-13. https://cran.r-project.org/web/packages/rjags/index.html (accessed 6 September 2017).

$\mathrm{R}$ Core Team, 2014, R-A language and environment for statistical computing: Vienna, Austria, $\mathrm{R}$ Foundation for Statistical Computing.

Royle, J.A., and Dorazio, R.M., 2008, Hierarchical modeling and inference in ecology-The analysis of data from populations, metapopulations, and communities: London, Academic Press, 464 p. 
Spens, J., Evans, A.R., Halfmaerten, D., Knudsen, S.W., Sengupta, M.E., Mak, S.S.T., Sigsgaard, E.E., and Hellström, M., 2017, Comparison of capture and storage methods for aqueous macrobial eDNA using an optimized extraction protocol-Advantage of enclosed filter: Methods in Ecology and Evolution, v. 8, no. 5, p 635-645.

Strickler, K.M., Fremier, A.K., and Goldberg, C.S., 2015, Quantifying effects of UV-B, temperature, and $\mathrm{pH}$ on eDNA degradation in aquatic microcosms: Biological Conservation, v. 183, p. 85-92.

Turner, C.R., Barnes, M.A., Xu, C.C.Y., Jones, S.E., Jerde, C.L., and Lodge, D.M., 2014, Particle size distribution and optimal capture of aqueous macrobial eDNA: Methods in Ecology and Evolution, $\mathrm{v}$. 5, p. 676-684.

U.S. Fish and Wildlife Service, 1993, Endangered and threatened wildlife and plants - Determination of threatened status for the giant garter snake: Federal Register, v. 58, p. 54,053-54,066.

U.S. Fish and Wildlife Service, 1999, Draft recovery plan for the giant garter snake (Thamnophis gigas): U.S. Fish and Wildlife Service, Sacramento, California.

Williams, K.E., Huyvaert, K.P., and Piaggio, A.J, 2017, Clearing muddied waters - Capture of environmental DNA from turbid waters, PLos ONE v. 12, p. e0179282.

Wood, D.A., Halstead, B.J., Casazza, M.L., Hansen, E.C., Wylie, G.D, and Vandergast, A.G., 2015, Defining population structure and genetic signatures of decline in the giant gartersnake (Thamnophis gigas)_-Implications for conserving threatened species within highly altered landscapes: Conservation Genetics, v. 16, p. 1,025-1,039.

Wylie, G.D., Casazza, M.L., Gregory, C.J., and Halstead, B.J., 2010, Abundance and sexual size dimorphism of the giant gartersnake (Thamnophis gigas) in the Sacramento Valley of California: Journal of Herpetology, v. 44, p. 94-103. 
Appendix 1. Sequence Alignment of Thamnophis gigas and Closely Related and (or) Potentially Sympatric Snake Species for the Nicotinamide Adenine Dinucleotide (Reduced Form)(NADH) Dehydrogenase Subunit 4 (ND4) and Primers/Probe Set Used in Quantitative Polymerase Chain Reaction (qPCR) Assays

[Sequences highlighted in blue indicate the conserved nucleotides across the different species' sequence alignments]

T. ATRATUS_ND4
T. COUCHII_ND4
T. ELEGANS_ND4
T. GIGAS_ND 4
T. SIRTALIS_ND4
N. FASCIATA_ND4
ND4_Probe
ND4_FORWARD
ND4_REVERSE
Consensus

T. ATRATUS_ND4

T. COUCHII_ND4

T. ELEGANS ${ }^{-}$ND4

THGI_ND 4

T. SİRTALIS_ND 4

N. FASCIATA ND4

ND4_Probe

ND4 FORWARD

ND4_REVERSE

Consēnsus

T. ATRATUS_ND4

T. COUCHII ND4

T. ELEGANS ND4

THGI ND4

T.SIRTALIS_ND4

N.FASCIATA_ND4

ND4 Probe

ND4_FORWARD

ND 4_REVERSE

Consensus
1

(1) GCAGCAATCCTTTTAAAACTGGGGGGCTATGGTATTATCCGGATAATACAAACTCTCCCAACAATAAAAACAGACGCGTT

(1) GCAGCAATCCTTTTAAAACTAGGAGGCTATGGTATTATCCGAATAATACAAACTCTCCCAACAATAAAAACAGACGCGTT

(1) GCAGCARTCCTTTTAAAACTGGGAGGCTATGGYATTATCCGAATAATACAAACTCTCCCAACAATAAAAACAGACGCGTT

(1) GCAGCAATCCTTTTAAAACTAGGAGGCTACGGYATTATCCGAATAATACAAACCCTCCCAACAATAAAAACAGACGCGTT

(1) GCAGCAATCCTTTTAAAACTRGGGGGCTACGGYATTATCCGAATAACACAAACTCTCCCAACAATAAAAACGGACACATT

(1) GCAGCAATCCTTTTAAAACTGGGGGGTTATGGTATTATCCGAATAACACAAACCCTCCCAACAATAAAAACAGACACATT

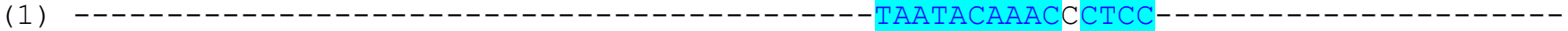

(1) -CAGCAATCCTTTTAAAACTAGGA-

(1)

GCAGCAATCCTTTTAAAACT Gg GgCTA Gg ATtATCCGAATAATACAAAC CTCCCAACAATAAAAACAGAC C TT
81

CCTTCCATTTATCGTCCTTGCCCTCTGAGGAGCAACACTGGCTAATCTTACCTGCTTACAACAAACAGACCTAAAATCCT

(81) CCTTCCATTTATCGTCCTTGCCCTCTGAGGAGCAACACTGGCTAATCTTACCTGCTTACAACAAACAGACCTAAAATCCT

(81) CCTACCATTTATCGTCCTTGCCCTTTGAGGAGCAACATTGGCTAATCTTACCTGCTTACAACAAACAGACCTAAAATCCT

(81) CCTTCCATTTATYGTCCTTGCCCTCTGAGGAGCAACACTGGCTAATCTTACCTGCTTACAACAAACAGACCTAAAATCCT

(81) TCTACCATTTATYATCCTCGCCCTTTGAGGAGCAMCATTAGCCAACCTCACTTGCCTACAACAAACAGATCTAAAGTCCC

(81) CCTACCATTTATCGTTCTTGCCCTCTGAGGAGCAACACTAGCTAACCTTACCTGCTTACAACAAACAGAYCTAAAATCCT

(81)

(81)

$(81)$

(81) CCT CCAT

CCT CCATTTAT GTCCTTGCCCTCTGAGGAGCAACACTGGCTAA CTTACCTGCTTACAACAAACAGA CTAAAATCCT 161

240

(161) TAATCGCATATTCATCTGTCAGTCATATAGGCCTTGTTATTTCTGCCATTATAATCCAAACACAATGAAGtCTGTCAGGA

(161) TAATCGCATATTCATCTGTTAGTCATATAGGCCTAGTCATTTCTGCCATTATAATCCAAACACAATGAAGTCTGTCGGGA

(161) TAATCGCATATTCATCTGTTAGYCATATRGGCCTAGTYATTTCYGCCATTATAATCCAAACACAATGAAGTCTGTCAGGA

(161) TAATCGCATATTCATCTGTCAGTCATATRGGCCTAGYCATTTCTGCCATTATAATCCAAACACAATGAAGTCTGTCAGGA

(161) TAATCGCATATTCATCTATCAGCCAYATAGGTTTGGTTATTTCCGCCATTATAATCCAAACACAATGAAGCCTATCAGGG

(161) TAATCGCATATTCATCCATCAGCCAYATRGGCCTRGTTATTTCCGCCATTATAATCCAAACACAATGAAGCCTATCCGGA

$(161)$

$(161)$

$(161)$

(161)

TAATCGCATATTCATCT T Ag CA AT GGCCT GT ATTTC GCCATTATAATCCAAACACAATGAAg CT TC GGA 
T. ATRATUS ND4 (241) ACCATAGCCCTAATAATCGCCCACGGATTTACCTCATCAGCACTTTTCTGCCTAGCTAACACCTCCTATGAACGAACAAA T. COUCHII_ND4 (241) GCCATAACCCTAATAATCGCCCACGGATTTACCTCATCAGCACTTTTCTGCCTAGCTAACACCTCCTATGAACGAACAAA T. ELEGANS ${ }^{-}$ND4 (241) ACCATAGCCYTAATAATCGCCCACGGATTYACCTCATCAGCACTTTTCTGYCTAGCTAACACCTCCTATGAACGAACAAA T.SIRTALIS_ND4 (241) ACCATAGCCCTAATAATCGCCCACGGATTCACCTCATCAGCACTCTTCTGCCTAGCCAACACYTCCTATGAACGAACAAA

(241) ACCATGGCCCTAATAATCGCCCAYGGATTTACCTCATCAGCACTCTTCTGCCTAGCCAATACTTCCTATGAACGAACAAA ND4 Probe ND 4 FORWARD ND4_REVERSE Cons̄ensus (241) (241)

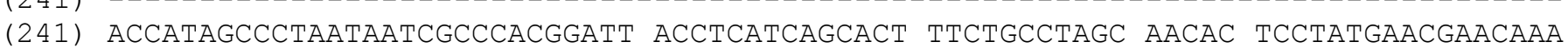
321

T. ATRATUS_ND4 (321) AACTCGAATTATAATCCTCACACGAGGTCTACACAACATCCTTCCTATAATAACCACCTGATGGCTATTAATCAATCTAA T. COUCHII ${ }^{-}$ND4 (321) AACTCGAATTATAATCCTTACACGAGGACTACACAACATCCTTCCTATAATAACCACCTGATGACTATTAATCAATCTAA T. ELEGANS_ND4 (321) AACYCGAATTATAATCCTCACACGAGGRCTACACAACATCCTTCCTATAATAACCACCTGATGRYTATTAATCAATYTAA THGI ND4 - (321) AACTCGAATTTTAATCCTCACACGAGGACTACACAACATCCTTCCTATAATAACCACCTGATGACTATTAATCAATCTAA T.SIRTALIS ND4 (321) AACCCGAATTATAATCCTCACGCGAGGACTACACAAYATCCTTCCCATAATAACCACCTGGTGGTTATTAATTAACCTAA N.FASCIATA_ND4 (321) AACCCGAATTATAATCCTCACACGAGGACTACACAAYATCCTTCCCATAATAACCRCCTGRTGRTTRCTAATTAACCTAA ND4 Probe ND4_FORWARD ND4_REVERSE Consensus 401

T. ATRATUS ND4 (401) TAMACATTGCTACTCCCCCCACCATAAACTTCACAGGCGAGTTATTAATCGCCTCATCCCTATTCAACTGATGTCCCACA T. COUCHII_ND4 (401) TAMACATTGCTACTCCCCCCACCATAAACTTCACAGGCGAGTTATTAATCGCCTCATCCCTATTCAATTGATGTCCCACA T. ELEGANS ${ }^{-}$ND4 (401) TAMACATTGCTACTCCCCCCACCATAAACTTCACAGGCGAGTTATTAATCGCCTCATCCCTATTCAACTGATGTCCCACA THGI ND4 (401) TAMACATTGCTACTCCCCCCACCATAAACTTCACAGGCGAGTTATTAATCGCCTCATCCCTATTCAACTGATGTCCCACA T.SIRTALIS_ND4 (401) TAMATATTGCCACTCCCCCCAGCATAAACTTCACAGGCGAATTATTAATCGCCTCATCCCTATTCAACTGATGTCCCACA N.FASCIATA_ND4 (401) TAMATATTGCCACCCCCCCCAGCATAAACTTCACAGGCGAATTAYTAATCGCCTCCTCCCTATTCAACTGATGYCCCACA ND4_Probe ND4 FORWARD ND4 REVERSE Consensus

$(401)$

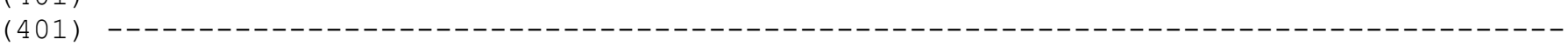

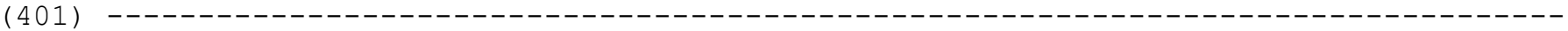

(401) TAMA ATTGC ACTCCCCCCA CATAAACTTCACAGGCGA tTATTAATCGCCTCATCCCTATtCAACTGATGTCCCACA 481

T. ATRATUS_ND4 (481) ACAATTATTATATTCGGACTATCTATACTAATCACAGCATCtTACTCTCTTCATATgTTCCTATCAACACAAATAAACCT T. COUCHII_ND4 (481) ACAATTATTATATTCGGACTATCTATACTAATCACAGCATCTTACTCTCTTCATATGTTCCTATCAACACAAATAAACCT T. ELEGANS ND4 (481) ACAATTATTATATTCGGACTATCTATACTAATCACAGCATCTTACTCTCTTCATATGTTCCTATCAACACAAATAAACCT THGI ND4 T.SIRTALIS_ND4

(481) ACAATTAYTATATTTGGACTATCTATACTAATCACAGCATCTTACTCTCTTCATATGTTCCTATCAACACAAATAAACCT N.FASCIATA_ND4

(481) ACAATTATCATGTTTGGATTATCAATACTAATTTCAGCATCCTACTCCCTCCACATATTCTTATCAACACAAATAAACCT ND4_Probe

(481)

ACAATTATTATATTTGGACTATCAATACTAATCACRGCATCCTACTCCCTYCACATATTTCTATCAACACAAATAAACCC 


\section{Appendix 1.-Continued}

ND4 FORWARD ND4_REVERSE Consensus

T. ATRATUS T. COUCHII ND4 T. ELEGANS ${ }^{-}$ND4 THGI ND4 T.SIRTALIS_ND4 N.FASCIATA-ND4 ND4 Probe ND4 FORWARD ND4 REVERSE

Consensus

T. ATRATUS ND4 T. COUCHII ${ }^{-} \mathrm{ND}$ T. ELEGANS ${ }^{-}$ND4 THGI_ND4 T.SIRTALIS_ND4 N.FASCIATA ND4 ND4 Probe ND4 FORWARD ND4 REVERSE Consensus

T. ATRATUS_ND4 T. COUCHII ND4 T. ELEGANS ND4 THGI ND4 T.SIRTALIS ND4 N.FASCIATA ND4 ND4 Probe ND 4 FORWARD ND4 REVERSE Consensus
(481)

(481) ACAATTATTATATT GGACTATC ATACTAATCACAGCATC TACTC CT CA AT TTCCTATCAACACAAATAAACCT 561

640

(561) CACACCATCAAACACCCCAATTCAACCCACACACTCACGAGAACACCTACTTATACTACTCCACACCCTACCACTTATCC (561) CACACCATCAAACGCCCCAATTCAACCCACACACTCACGAGAACACCTACTTATACTACTCCACACCCTACCACTTATCC (561) CACACCATCAAACACCCCYATTCAACCCACACACTCACGAGAACACCTACTTATACTACTCCACACCCTACCACTTATCC (561) CACACCATCAAACGCCCCAATTCAACCCACACATTCACGAGAACACCTACTTATACTCCTCCACACCCTACCACTTATCC (561) YACACCATCAAACACCCCTATTCAACCCACACATTCACGAGAGCACCTACTTATACTCCTCCACACCCTACCACTTATCC (561) CCСАСТАTCAAACACCCCCATTCAACCTTCACACTCACGAGAACAYCTACTTATACTACTCCACACCCTACCACTTATCC (561) ---------------------------------------------------------------------

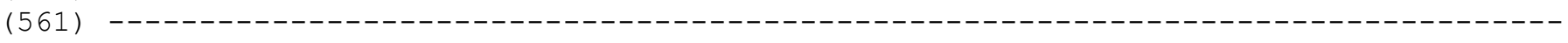
$(561)$

(561) CACACCATCAAAC CCCC ATTCAACCCACACA TCACGAGAACACCTACTTATACT CTCCACACCCTACCACTTATCC 641

(641) TGGTCTCCTTAAAACCCGAACTGGTAATTTAGTGTATGTAATTTAAATAAAATATCAAGCTGTGACCCTGACAATAGGAA GGTCTCCTTAAAACCCGAACTGGTAATTTAGTGTATGTAATTTAAATAAAATATCAAGCTGTGACCCTGACAATAGGAA TGGTCTCCTTAAAACCCGAACTGGTAATTTAGTGTATGTAATTTAAATAAAATATCAAGCTGTGACCCTGACAATAGGAA

(641) TGGTCTCCTTAAAACCCGAACTGGTAATTTAGTGTATGTAATTTAAATAAAATATCAAGCTGTGACCCTGACAATAGGAA

(641) TGGTCTCCTTAAAACCCGAACTGGTAATTTAGTGTATGTAATTTAAATAAAATATCAAGCTGTGACCCTGACAATAGGAA

(641) TGGTCTCCTTAAAACCCGAACTGGTAATTAAGTTATGTAATTTAAATAAAATATCAAGCTGTGACCCTGACAATAGGAA

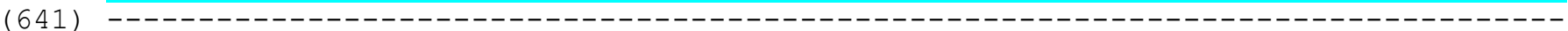

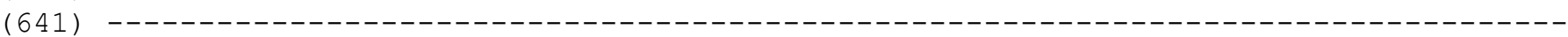

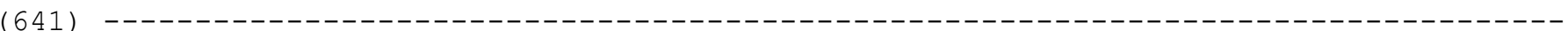

(641) TGgTCTCCTTAAAACCCGAACTGGTAATTAGTGTATGTAATTTAAATAAAATATCAAGCTGTGACCCTGACAATAGGAA 721

(721) TCCTCCTCATACACCAGAGGGCGCAATAAGACCTGCTAACTCTTAAACCCGGAAATAACAGCCGGCCCCCTCTACCAAAG

(721) TCCTCCTCATACACCAGAGGGCGCAATAAGACCTGCTAACTCTTAAACCCGGAAATAACAGCCGGCCCCCTCTACCAAAG

(721) TCCTCCTCATACACCAGAGGGCGCAATAAGACCTGCTAACTCTTAAACCCGGAAATAACAGCCGGCCCCCTCTACCAAAG

(721) TCCTCCTCATACACCAGAGGGCGCAATAAGACCTGCTAACTCTTAAACCCGGAAATAACAGCCGGCCCCCTCTACCAAAG

721) TCCTCCTCATACACCAGAGGGCGCAATAAGACCTGCTAACTCTTAAACCCGGAAATAACAGCCGGCCCCCTCTACCAAAG

(721) TCCTCCTCATACACCAGAGGGCGCAATAAGACCTGCTAACTCTTAAACCCGGAAATAACAGCCGGCCCCCTCTACCAAAG

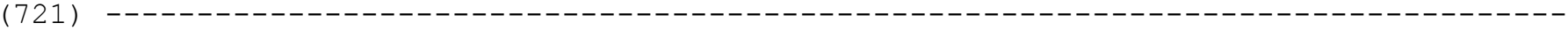

(721) --- - - - - - - - - - - - - - - - - - - - - - - - - - - - - - - - - - - - - - - - - - - - - - - - - - - - - - - -

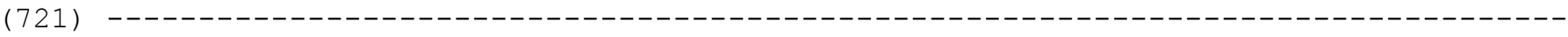

(721) TCCTCCTCATACACCAGAGGGCGCAATAAGACCTGCTAACTCTTAAACCCGGAAATAACAGCCGGCCCCCTCTACCAAAG 
Appendix 1.-Continued

801

T. ATRATUS_ND4 (801) GATAATAGTATTCCACTGGTCTTAGGCACCAAAATCYT

T. COUCHII ND4 (801) GATAATAGTATTCCACTGGTCTTAGGCACCAAAATCYT

T. ELEGANS_ND4 (801) GATAATAGTATTCCACTGGTCTTAGGCACCAAAATCYT

THGI_ND4 - $(801)$ GATAATAGTATTCCACTGGTCTTAGGCACCAAAATCYT

T.SIRTALIS ND4 (801) GATAATAGTATTCCACTGGTCTTAGGCACCAAAATCYT

N.FASCIATA_ND4 (801) GATAATAGTATTCCACTGGTCTTAGGCACCAAAATCYT

ND4 Probe

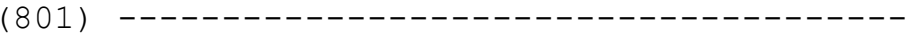

ND4 FORWARD

ND4 REVERSE

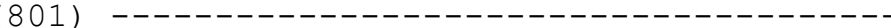

Consensus

(801) GAtAATAgTATTCCACTGGTCTTAgGCACCAAAATCYT 


\section{Appendix 2. Sequence Alignment of Nerodia fasciata and Potentially Sympatric Snake Species for the}

Nicotinamide Adenine Dinucleotide (Reduced Form) (NADH) Dehydrogenase Subunit 4 (ND4) and Primers/Probe Set for Use in Quantitative Polymerase Chain Reaction (qPCR) Assays, in the Central Valley, California

[Sequences highlighted in blue indicate the conserved nucleotides across the different species' sequence alignments]

T. ATRATUS_ND4

T. COUCHII-ND4

T. ELEGANS ND4

T. GIGAS_ND 4

T.SIRTALİS ND4

N.FASCIATA ND4

N.FASCIATA_ND4 F

N.FASCIATA Probe

N.FASCIATA_ND4_R

Consensus

T. ATRATUS ND4

T. COUCHII ND4

T. ELEGANS_ND4

T. GIGAS ND 4

T.SIRTALI S ND4

N. FASCIATA ND4

N.FASCIATA ND4 F

N.FASCIATA_Probe

N.FASCIATA ND4 R

Consensus

T. ATRATUS ND4

T. COUCHII_ND4

T. ELEGANS ND4

T. GIGAS ND 4

T.SIRTALIS_ND4

N.FASCIATA ND4

N.FASCIATA_ND4_F

N.FASCIATA Probe

N.FASCIATA_ND4_R

Consensus
(1) GCAGCAATCCTTTTAAAACTGgGgGgCTATGGTATTATCCGgATAATACAAACTCTCCCAACAATAAAAACAGACGCGTT

(1) GCAGCAATCCTTTTAAAACTAGGAGGCTATGGTATTATCCGAATAATACAAACTCTCCCAACAATAAAAACAGACGCGTT

(1) GCAGCARTCCTTTTAAAACTGGGAGGCTATGGYATTATCCGAATAATACAAACTCTCCCAACAATAAAAACAGACGCGTT

(1) GCAGCAATCCTTTTAAAACTAGGA GGCTACGGYATTATCCGAATAATACAAACCCTCCCAACAATAAAAACAGACGCGTT

(1) GCAGCAATCCTTTTAAAACTRGGGGGCTACGGYATTATCCGAATAACACAAACTCTCCCAACAATAAAAACGGACACATT

(1) GCAGCAATCCTTTTAAAACTGGGgGgTTATGGTATTATCCGAATAACACAAACCCTCCCAACAATAAAAACAGACACATT

(1)

(1)

(1) GCA 81

(81) CCTTCCATTTATCGTCCTTGCCCTCTGAGGAGCAACACTGGCTAATCTTACCTGCTTACAACAAACAGACCTAAAATCCT

(81) CCTTCCATTTATCGTCCTTGCCCTCTGAGGAGCAACACTGGCTAATCTTACCTGCTTACAACAAACAGACCTAAAATCCT

(81) CCTACCATTTATCGTCCTTGCCCTTTGAGGAGCAACATTGGCTAATCTTACCTGCTTACAACAAACAGACCTAAAATCCT

(81) CCTTCCATTTATYGTCCTTGCCCTCTGAGGAGCAACACTGGCTAATCTTACCTGCTTACAACAAACAGACCTAAAATCCT

(81) TCTACCATTTATYATCCTCGCCCTTTGAGGAGCAMCATTAGCCAACCTCACTTGCCTACAACAAACAGATCTAAAGTCCC

(81) CCTACCATTTATCGTTCTTGCCCTCTGAGGAGCAACACTAGCTAACCTTACCTGCTTACAACAAACAGAYCTAAAATCCT

(81)

(81)

$(81)$

(81)

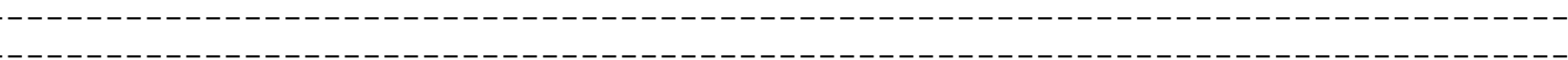

CCT CCATTTAT GTCCTTGCCCT TGAGGAGCAACA T GCTAA CTTACCTGCTTACAACAAACAGA CTAAAATCCT 161

240

(161) TAATCGCATATTCATCTGTCAGTCATATAGGCCTTGTTATTTCTGCCATTATAATCCAAACACAATGAAGTCTGTCAGGA

(161) TAATCGCATATTCATCTGTTAGTCATATAGGCCTAGTCATTTCTGCCATTATAATCCAAACACAATGAAGTCTGTCGGGA

(161) TAATCGCATATTCATCTGTTAGYCATATRGGCCTAGTYATTTCYGCCATTATAATCCAAACACAATGAAGTCTGTCAGGA

(161) TAATCGCATATTCATCTGTCAGTCATATRGGCCTAGYCATTTCTGCCATTATAATCCAAACACAATGAAGTCTGTCAGGA

(161) TAATCGCATATTCATCTATCAGCCAYATAGGTTTGGTTATTTCCGCCATTATAATCCAAACACAATGAAGCCTATCAGGG

(161) TAATCGCATATTCATCCATCAGCCAYATRGGCCTRGTTATTTCCGCCATTATAATCCAAACACAATGAAGCCTATCCGGA

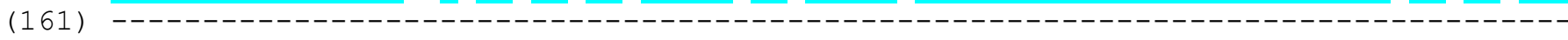

(161)

(161)

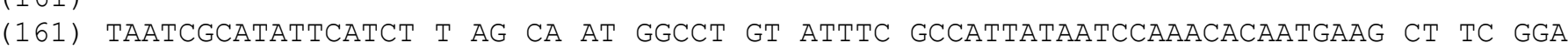




\section{Appendix 2.-Continued}

241

T. ATRATUS_ND4

T. COUCHII ND4

T. ELEGANS ND 4

T. GIGAS ND 4

T.SIRTALIS ND4

N.FASCIATA_ND4

N.FASCIATA_ND4_F

N.FASCIATA_Probe

N.FASCIATA_ND4_R

Consensus

T. ATRATUS ND4

T. COUCHII_ND4

T. ELEGANS ND4

T. GIGAS ND 4

T.SIRTALIS_ND4

N. FASCIATA ND4

N.FASCIATA_ND4_F

N.FASCIATA_Prōe N. FASCIATA ND4 R

Consensus

T. ATRATUS_ND4

T. COUCHII_ND4

T. ELEGANS ND4

T. GIGAS_ND 4

T.SIRTALISSND 4

N.FASCIATA_ND4

N.FASCIATA_ND4_F

N.FASCIATA Probe

N.FASCIATA_ND4_R (401)

Consensus
(241) ACCATAGCCCTAATAATCGCCCACGGATTTACCTCATCAGCACTTTTCTGCCTAGCTAACACCTCCTATGAACGAACAAA

(241) GCCATAACCCTAATAATCGCCCACGGATTTACCTCATCAGCACTTTTCTGCCTAGCTAACACCTCCTATGAACGAACAAA

(241) ACCATAGCCYTAATAATCGCCCACGGATTYACCTCATCAGCACTTTTCTGYCTAGCTAACACCTCCTATGAACGAACAAA

(241) ACCATAGCCCTAATAATCGCTCACGGATTTACCTCATCAGCACTTTTCTGCCTAGCCAACACCTCCTATGAACGAACAAA

(241) ACCATAGCCCTAATAATCGCCCACGGATTCACCTCATCAGCACTCTTCTGCCTAGCCAACACYTCCTATGAACGAACAAA

(241) ACCATGGCCCTAATAATCGCCCAYGGATTTACCTCATCAGCACTCTTCTGCCTAGCCAATACTTCCTATGAACGAACAAA

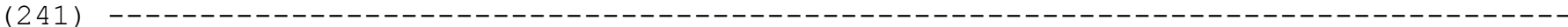

(241) --

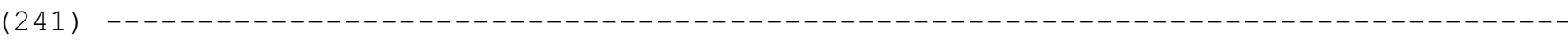

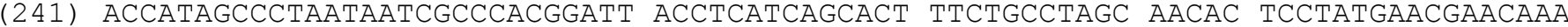
321

400

(321) AACTCGAATTATAATCCTCACACGAGGTCTACACAACATCCTTCCTATAATAACCACCTGATGGCTATTAATCAATCTAA

(321) AACTCGAATTATAATCCTTACACGAGGACTACACAACATCCTTCCTATAATAACCACCTGATGACTATTAATCAATCTAA

(321) AACYCGAATTATAATCCTCACACGAGGRCTACACAACATCCTTCCTATAATAACCACCTGATGRYTATTAATCAATYTAA

(321) AACTCGAATTTTAATCCTCACACGAGGACTACACAACATCCTTCCTATAATAACCACCTGATGACTATTAATCAATCTAA

(321) AACCCGAATTATAATCCTCACGCGAGGACTACACAAYATCCTTCCCATAATAACCACCTGGTGGTTATTAATTAACCTAA

(321) AACCCGAATTATAATCCTCACACGAGGACTACACAAYATCCTTCCCATAATAACCRCCTGRTGRTTRCTAATTAACCTAA

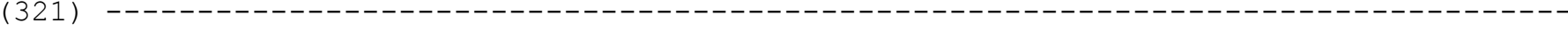

(321) --- - - - - - - - - - - - - - - - - - - - - - - - - - - - - - - - - - - - - - - - - - - - - - - - - - - - - - - - -

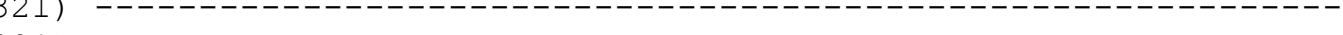

(321) AAC CGAATTATAATCCTCACACGAgG CTACACAA ATCCTTCC ATAATAACCACCTG TG TATTAAT AA CTAA

(401) TAMACATTGCTACTCCCCCCACCATAAACTTCACAGGCGAGTTATTAATCGCCTCATCCCTATTCAACTGATGTCCCACA

(401) TAMACATTGCTACTCCCCCCACCATAAACTTCACAGGCGAGTTATTAATCGCCTCATCCCTATTCAATTGATGTCCCACA

(401) TAMACATTGCTACTCCCCCCACCATAAACTTCACAGGCGAGTTATTAATCGCCTCATCCCTATTCAACTGATGTCCCACA

(401) TAMACATTGCTACTCCCCCCACCATAAACTTCACAGGCGAGTTATTAATCGCCTCATCCCTATTCAACTGATGTCCCACA

(401) TAMATATTGCCACTCCCCCCAGCATAAACTTCACAGGCGAATTATTAATCGCCTCATCCCTATTCAACTGATGTCCCACA

(401) TAMATATTGCCACCCCCCCCAGCATAAACTTCACAGGCGAATTAYTAATCGCCTCCTCCCTATTCAACTGATGYCCCACA ( 401 ) ----

(401) ---

(401) TAMA ATTGC ACTCCCCCCA CATAAACTTCACAGGCGA TTATTAATCGCCTCATCCCTATTCAACTGATGTCCCACA 


\section{Appendix 2.-Continued}

481

T. ATRATUS_ND4

T. COUCHII ND4

T. ELEGANS ND4

T. GIGAS_ND 4

T.SIRTALIS ND4

N.FASCIATA_ND4

N.FASCIATA_ND4_F

N.FASCIATA_Probe

N.FASCIATA_ND4_R

Consensus

T. ATRATUS ND4

T. COUCHII_ND4

T. ELEGANS ND4

T. GIGAS ND 4

T.SIRTALIS_ND4

N.FASCIATA_ND4

N.FASCIATA_ND4_E

N.FASCIATA_Probe N.FASCIATA ND4 R

Consensus

T. ATRATUS_ND4

T. COUCHII ND4

T. ELEGANS ND4

T. GIGAS_ND 4

T.SIRTALISSND 4

N.FASCIATA_ND4

N.FASCIATA_ND4_F

N.FASCIATA Probe

N.FASCIATA_ND4_R

Consensus
(481) ACAATTATTATATTCGGACTATCtATACTAATCACAGCATCTTACTCTCTTCATATgTTCCTATCAACACAAATAAACCT

(481) ACAATTATTATATTCGGACTATCTATACTAATCACAGCATCTTACTCTCTTCATATGTTCCTATCAACACAAATAAACCT

(481) ACAATTATTATATTCGGACTATCTATACTAATCACAGCATCTTACTCTCTTCATATGTTCCTATCAACACAAATAAACCT

(481) ACAATTAYTATATTTGGACTATCTATACTAATCACAGCATCTTACTCTCTTCATATGTTCCTATCAACACAAATAAACCT

(481) ACAATTATCATGTTTGGATTATCAATACTAATTTCAGCATCCTACTCCCTCCACATATTCTTATCAACACAAATAAACCT

(481) ACAATTATTATATTTGGACTATCAATACTAATCACRGCATCCTACTCCCTYCACATATTTCTATCAACACAAATAAACCC

(481) --

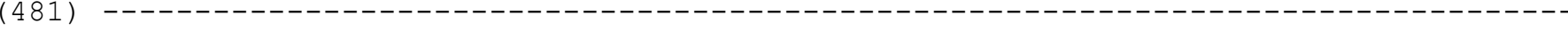

(481) ACAATTATTATATT GGACTATC ATACTAATCACAGCATC TACTC CT CA AT TTCCTATCAACACAAATAAACCT 561

640

(561) CACACCATCAAACACCCCAATTCAACCCACACACTCACGAGAACACCTACTTATACTACTCCACACCCTACCACTTATCC

(561) CACACCATCAAACGCCCCAATTCAACCCACACACTCACGAGAACACCTACTTATACTACTCCACACCCTACCACTTATCC

(561) CACACCATCAAACACCCCYATTCAACCCACACACTCACGAGAACACCTACTTATACTACTCCACACCCTACCACTTATCC

(561) CACACCATCAAACGCCCCAATTCAACCCACACATTCACGAGAACACCTACTTATACTCCTCCACACCCTACCACTTATCC

(561) YACACCATCAAACACCCCTATTCAACCCACACATTCACGAGAGCACCTACTTATACTCCTCCACACCCTACCACTTATCC

(561) CCCACTATCAAACACCCCCATTCAACCTTCACACTCACGAGAACAYCTACTTATACTACTCCACACCCTACCACTTATCC

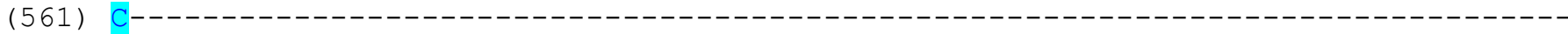

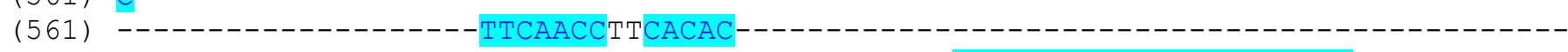

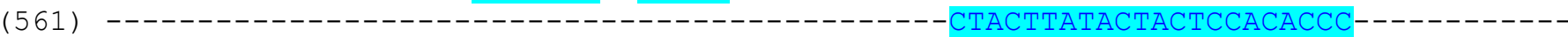

(561) CACACCATCAAAC CCCC ATTCAACCCACACACTCACGAGAACACCTACTTATACTACTCCACACCCTACCACTTATCC 641

720

(641) TGGTCTCCTTAAAACCCGAACTGGTAATTTAGTGTATGTAATTTAAATAAAATATCAAGCTGTGACCCTGACAATAGGAA

(641) TGGTCTCCTTAAAACCCGAACTGGTAATTTAGTGTATGTAATTTAAATAAAATATCAAGCTGTGACCCTGACAATAGGAA

(641) TGGTCTCCTTAAAACCCGAACTGGTAATTTAGTGTATGTAATTTAAATAAAATATCAAGCTGTGACCCTGACAATAGGAA

(641) TGGTCTCCTTAAAACCCGAACTGGTAATTTAGTGTATGTAATTTAAATAAAATATCAAGCTGTGACCCTGACAATAGGAA

(641) TGGTCTCCTTAAAACCCGAACTGGTAATTTAGTGTATGTAATTTAAATAAAATATCAAGCTGTGACCCTGACAATAGGAA

(641) TGGTCTCCTTAAAACCCGAACTGGTAATTTAGTGTATGTAATTTAAATAAAATATCAAGCTGTGACCCTGACAATAGGAA

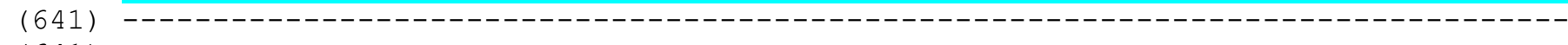

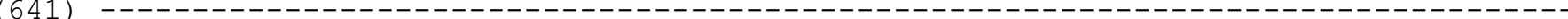

(641) TGGTCTCCTTAAAACCCGAACTGGTAATTTAGTGTATGTAATTTAAATAAAATATCAAGCTGTGACCCTGACAATAGGA 


\section{Appendix 2.-Continued}

721

800

T. ATRATUS_ND4

T. COUCHII_ND4

T. ELEGANS ND4

T. GIGAS N $\bar{D} 4$

T.SIRTALIS_ND4

N.FASCIATA_ND4

N.FASCIATA ND4_F (721)

N.FASCIATA_Probe (721)

N.FASCIATA_ND4_R (721)
Consensus
(721)

(721) TCCTCCTCATACACCAGAGGGCGCAATAAGACCTGCTAACTCTTAAACCCGGAAATAACAGCCGGCCCCCTCTACCAAAG

(721) TCCTCCTCATACACCAGAGGGCGCAATAAGACCTGCTAACTCTTAAACCCGGAAATAACAGCCGGCCCCCTCTACCAAAG

(721) TCCTCCTCATACACCAGAGGGCGCAATAAGACCTGCTAACTCTTAAACCCGGAAATAACAGCCGGCCCCCTCTACCAAAG

(721) TCCTCCTCATACACCAGAGGGCGCAATAAGACCTGCTAACTCTTAAACCCGGAAATAACAGCCGGCCCCCTCTACCAAAG

(721) TCCTCCTCATACACCAGAGGGCGCAATAAGACCTGCTAACTCTTAAACCCGGAAATAACAGCCGGCCCCCTCTACCAAAG

T. ATRATUS ND4

T. COUCHII-ND4

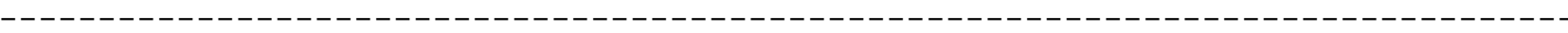

TCCTCCTCATACACCAGAGGGCGCAATAAGACCTGCTAACTCTTAAACCCGGAAATAACAGCCGGCCCCCTCTACCAAAG 801

838

(801) GATAATAGTATTCCACTGGTCTTAGGCACCAAAATCYT

(801) GATAATAGTATTCCACTGGTCTTAGGCACCAAAATCYT

T. ELEGANS_ND4 (801) GATAATAGTATTCCACTGGTCTTAGGCACCAAAATCYT

T. GIGAS ND 4

(801) GATAATAGTATTCCACTGGTCTTAGGCACCAAAATCYT

T.SIRTALIS_ND4 (801) GATAATAGTATTCCACTGGTCTTAGGCACCAAAATCYT

N.FASCIATA_ND4 (801) GATAATAGTATTCCACTGGTCTTAGGCACCAAAATCYT

N.FASCIATA ND4 F (801) - - - - - - -

N.FASCIATA_Probe (801) -------------------------------

N.FASCIATA ND4 R (801) -----------------------------------

Consensus (801) GATAATAGTATTCCACTGGTCTTAGGCACCAAAATCYT 
Appendix 3. Sequence Alignment of Thamnophis gigas and Potentially Sympatric Snake Species for the Cytochrome b (Cytb) Gene and Primers/Probe Sets for Use in Quantitative Polymerase Chain Reaction (qPCR) Assays, in the Central Valley, California

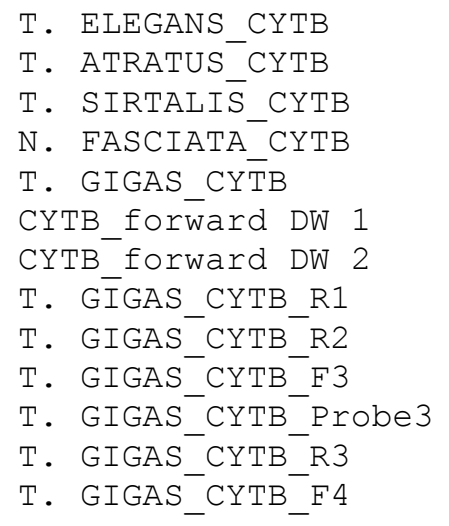

T. ELEGANS_CYTB T. ATRATUS CYTB T. SIRTALIS CYTB NEFA CYTB T. GI $G A S$ CYTB CYTB forward DW 1 CYTB forward DW 2 T. GIGAS CYTB R1 T. GIGAS_CYTB_R2 T. GIGAS CYTB F3 T. GIGAS CYTB Probe3 T. GIGAS ${ }^{-} \mathrm{CYTB}^{-} \mathrm{R} 3$ T. GIGAS_CYTB_F4
(1) ATGCCCAACCAACACACACTTCTATTATTCAACCTATTACCAGTAGGACTAAACATCTCAGCATGATGAAACTTTGGATC

(1) ATGCCCAACCAACACACACTTCTATTATTCAACCTATTACCAGTAGGACTAAACATCTCAACTTGATGAAACTTTGGGTC

(1) ATGTCCAACCAACATACACTTCTATTATTCAACCTACTACCTGTGGGACTAAACATTTCAACCTGATGAAATTTTGGGTC

(1) ATGCCCAACCAACACACACTTCTATTATTCAACCTATTACCAGTAGGACTAAACATCTCAACTTGATGAAACTTTGGGTC

(1) ATGCCCAACCAACACACACTTCTATTATTCAACCTATTACCAGTAGGACTAAACATCTCAACTTGATGAAACTTTGGGTC

(1)

(1)

(1)

(1)

(1)

(1)

(1)

(1)

81

(81) CATACTACTAACCTGCTCAgCCCTACAAATCATtACAGGATTCTTCCTAGCAATCCACTATACAGCCAACATTAACCTAG

(81) TATACTACTAACCTGCTCAGCACTACAAATCATTACAGGATTCTTTCTAGCAATCCACTATACAGCTAACATCAACCTAG

(81) TATGCTATTAACCTGCTCAGCACTACAAATCATTACAGGGTTCTTTCTAGCAATCCACTATACAGCCAACATTAACCTAG

(81) TATACTACTAACCTGCTCAgCACTACAAATCATtACAGGATTCTTTCTAGCAATCCACTATACAGCTAATATCAACCTAG

(81) TATACTACTAACCTGCTCAGCACTACAAATCATtACAGGATTCTTTCTAGCAATCCACTATACAGCTAACATCAACCTAG

(81)

$(81)$

$(81)$

(81)

$(81)$

(81)

(81)

(81) 


\section{Appendix 3.-Continued}

T. ELEGANS CYTB

T. ATRATUS CYTB

T. SIRTALIS__CYTB

NEFA CYTB

T. GIGAS_CYTB

CYTB forward DW 1

CYTB forward DW 2

T. GIGAS_CYTB_RI

T. GIGAS ${ }^{-}$CYTB ${ }^{-}$R2

T. GIGAS_CYTB_F3

T. GIGAS CYTB Probe3

T. GIGAS_CYTB-R3

T. GIGAS_CYTB_F4

T. ELEGANS_CYTB

T. ATRATUS_CYTB

T. SIRTALI $\bar{S} \_$CYTB

NEFA CYTB

T. GIGAS_CYTB

CYTB_forward DW 1

CYTB forward DW 2

T. GĪGAS_CYTB_R1

T. GIGAS CYTB R2

T. GIGAS_CYTB_F3

T. GIGAS_CYTB-Probe3

T. GIGAS CYTB R3

T. GIGAS_CYTB_F4

T. ELEGANS_CYTB

T. ATRATUS CYTB

T. SIRTALIS CYTB

NEFA CYTB

T. GIGAS CYTB

CYTB forward DW 1

CYTB forward DW 2

T. GIGAS CYTB R1

T. GIGAS_CYTB-R2

T. GIGAS ${ }^{-} \mathrm{CYTB}^{-} \mathrm{F} 3$

T. GIGAS_CYTB-Probe3

T. GIGAS CYTB R3

T. GIGAS_CYTB_F4
161

240

(161) CCTTTTCATCCATCATCCATATTACACGTGATGTACCATATGGGTGAATCATACAAAACACCCATGCAATCGGCGCATCC

(161) CCTTTTCATCCATCATCCACATTACACGTGACGTACCATATGGATGAATCATACAAAACACCCATGCAATCGGCGCATCC

(161) CCTTCTCATCTATTATCCACATTACACGTGACGTACCATACGGATGAATTATACAAAATACCCATGCAATCGGCGCATCC

(161) CCTTCTCATCTATCATCCACATCACCCGCGACGTKCCATATGGATGAATTATACAAAACACCCATGCAATCGGCGCATCC

(161) CCTTTTCATCCATCATCCATATTACACGTGACGTACCATATGGATGAATCATACAAAACACCCATGCAATCGGCGCATCC

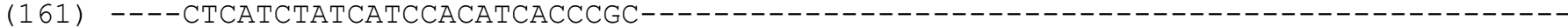

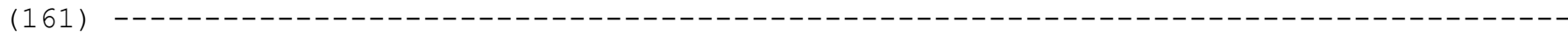

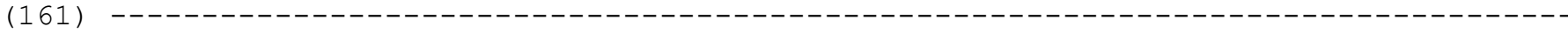

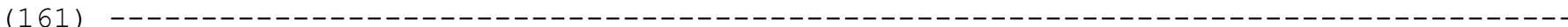

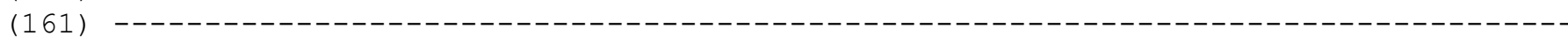

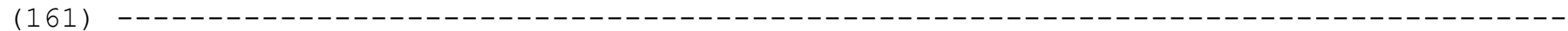

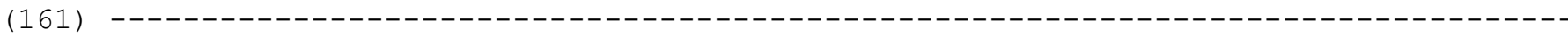

(1)

241

320

(241) AтAтTтTтAтTTGCATtTACATTCACATCGCACGAGGCCTCTACTACGGCTCCTACTTAAACAAAGAAGTCTGACTATC

(241) ATATTTTTTATTTGCATCTACATTCACATCGCACGAGGRCTCTACTACGGCTCCTACTTAAACAAAGAAGTCTGACTATC

(241) ctgTTTTTtATCTGtATtTATATTCACATTGCACGGGGACTCTACTATGGCTCCTACTTAAACAAAGAAGTRTGACTATC

(241) ATGTTTTTtATTTGCATCTACATTCACATTGCACGAGGACTTTATTACGGCTCCTATCTTAACAAAGAAGTCTGACTATC

(241) ATGTTTTTCATTTGCATCTACATTCACATCGCACGAGGGCTCTACTACGGCTCCTACTTAAACAAAGAAGTCTGACTATC

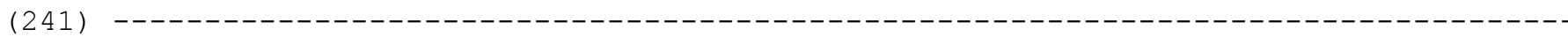

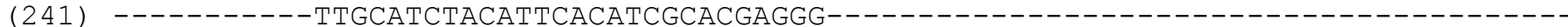

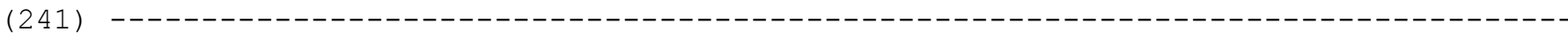

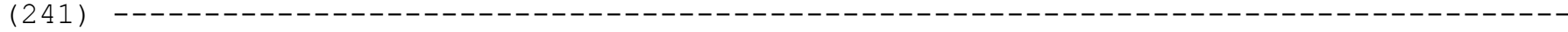

(241) --- - - - - - - - - - - - - - - - - - - - - - - - - - - - - - - - - - - - - - - - - - - - - - - - - - - - - - - -

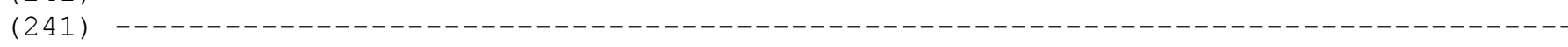

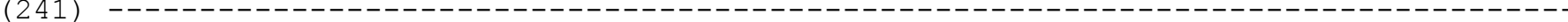

(241)

321

400

(321) CGGAACCACCCTCTTAATTATCCTAATAGCTACAGCCTTCTTCGGCTATGTCCTCCCATGAGGACAAATATCATTCTGAG

(321) CGGAACCACCCTTCTAATTATCCTAATAGCCACAGCCTTCTTTGGCTATGTCCTCCCATGAGGACAAATATCATTCTGAG

(321) AGGAACTACCCTTTTAATCRYTCTAATAGCCACAGCCTTCTTTGGCTATGTCCTCCCATGAGGACAAATATCATTCTGAG

(321) CGGAACCACCCTTTTAATCACCCTAATAGCCACAGCCTTCTTTGGCTACGTCCTCCCATGAGGACAAATATCATTCTGAG

(321) CGGAACCACCCTTCTAATTATCCTAATAGCCACAGCCTTCTTCGGCTATGTCCTCCCATGAGGACAAATATCATTCTGAG

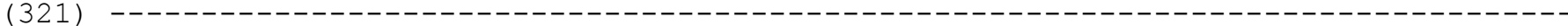

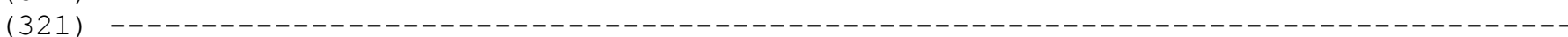

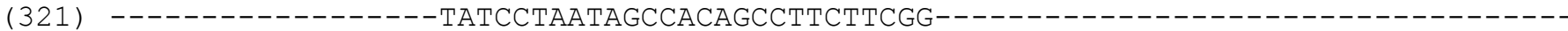

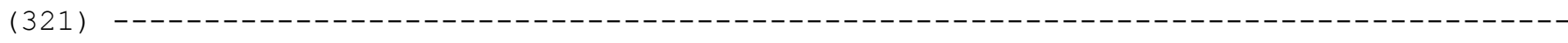

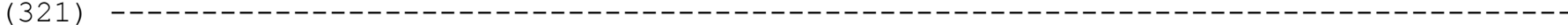

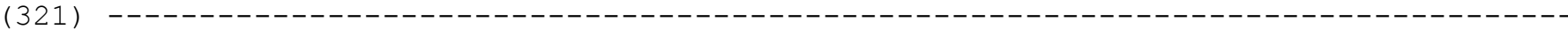

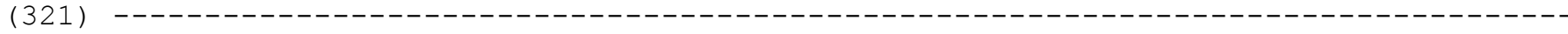

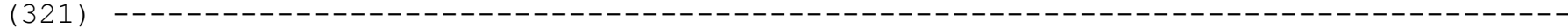




\section{Appendix 3.-Continued}

T. ELEGANS CYTB

T. ATRATUS CYTB

T. SIRTALIS__CYTB

NEFA CYTB

T. GIGAS_CYTB

CYTB forward DW 1

CYTB forward DW 2

T. GIGAS_CYTB_RI

T. GIGAS ${ }^{-} \mathrm{CYTB}^{-} \mathrm{R} 2$

T. GIGAS_CYTB_F3

T. GIGAS CYTB Probe 3

T. GIGAS_CYTB-R3

T. GIGAS_CYTB_F4

T. ELEGANS CYTB

T. ATRATUS_CYTB

T. SIRTALIS_CYTB

NEFA_CYTB

T. GIGAS CYTB

CYTB forward DW 1

CYTB forward DW 2

T. GIGAS_CYTB_R1

T. GIGAS CYTB R2

T. GIGAS_CYTB_F3

T. GIGAS_CYTB_Probe3

T. GIGAS_CYTB-R3

T. GIGAS_CYTB_E4

T. ELEGANS CYTB

T. ATRATUS CYTB

T. SIRTALIS_CYTB

NEFA CYTB

T. GI GAS CYTB

CYTB forward DW 1

CYTB forward DW 2

T. GĪGAS_CYTB_R1

T. GIGAS ${ }^{-}$CYTB R2

T. GIGAS CYTB F3

T. GIGAS_CYTB_Probe3

T. GIGAS CYTB R3

T. GIGAS_CYTB_F4
401

480

(401) CAGCAACTGTAATTACAAACCTACTAACCGCCGTACCATATCTCGGAAACAGCCTCACCACCTGACTTTGAGGCGGATTC

(401) CAGCAACTGTAATTACAAACCTACTAACCGCCGTACCATATCTCGGAAACAGCCTCACCACCTGACTTTGAGGCGGATTC

(401) CAGCAACAGTAATTACAAACCTACTAACCGCCGTACCTTACCTAGGAAACACCCTCACAACCTGACTYTGGGGCGGATTC

(401) CAGCAACAGTAATCACAAACCTACTAACCGCCGTACCATACCTTGGAAACAGTCTCACAACCTGACTCTGAGGCGGATTC

(401) CAGCAACTGTAATTACAAACCTACTAACCGCCGTACCATATCTCGGAAACAGCCTCACCACCTGACTTTGAGGCGGATTC

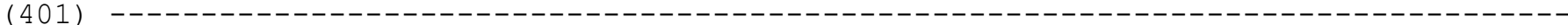

$(401)--------------------------------------------------------------------$

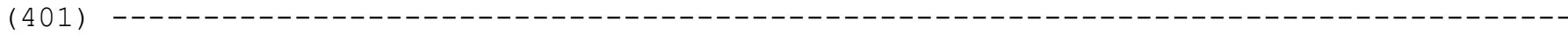

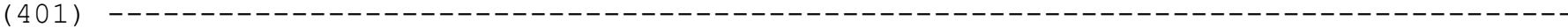

( 401 ) -------------------------------------------GCCTCACCACCTGACTTTGA--------

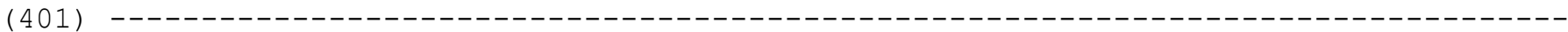

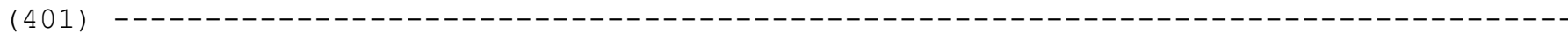

$(401)$

481

560

(481) TCAATTAATGACCCGACCCTAACACGATTCTTTGCCCTCCACTTTATCCTACCATTTGCCATTATCTCACTATCTTCCAT

(481) TCAATTAATGACCCCACCCTAACACGATTCTTTGCCCTTCACTTTATCCTACCATTTGCCATTATCTCACTATCCTCCAT

(481) TCAATTAATGATCCAACCCTAACACGATTCTTTGCTCTCCATTTCATTCTACCATTTGCCATTATTTCATTATCCTCCAT

(481) TCAATTAATGACCCAACCCTAACACGATTCTTTGCCCTACATTTTATCCTTCCTTTTGCCATCATCTCACTATCTTCCAT

(481) TCAATTAATGACCCGACCTTAACACGATTCTTTGCCCTCCACTTTATCCTACCATTTGCCATTATCTCACTATCTTCCAT

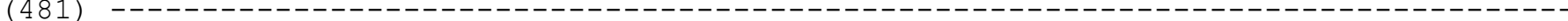

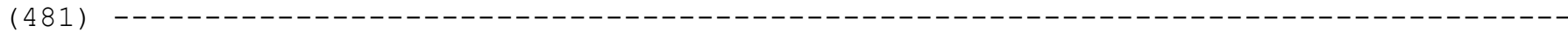

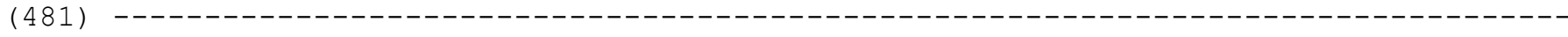

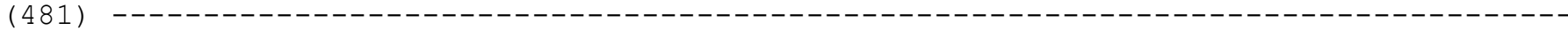

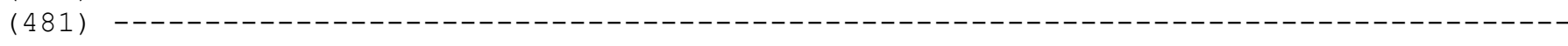

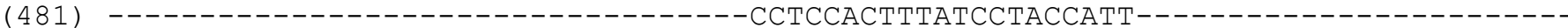

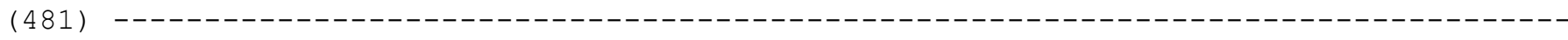

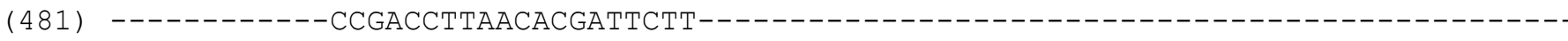

561

640

(561) CCACATTATGCTACTACACACCGAAGGCTCAAGCAACCCACTAGGAACAAACTCAGACATCGACAAAATCCCATTCCATC

(561) CCACATTATGCTACTACACACCGAAGGCTCAAGCAACCCACTAGGAACAAACTCAGACATCGACAAAATCCCATTCCATC

(561) CCACATTATACTATTACACACTGAAGGCTCAAGCAACCCACTAGGAACAAACTCAGACATTGACAAAATCCCATTCTACC

(561) TCACATCATACTACTACACACCGAGGGTTCAAGCAACCCCTTAGGAACAAACTCAGACATTGACAAAATCCCATTCCACC

(561) CCACATCATGCTACTACACACCGAAGGCTCAAGCAACCCACTAGGAACAAACTCAGACATTGACAAAATCCCATTCCATC

(561) ----------------------------------------------------------------------------

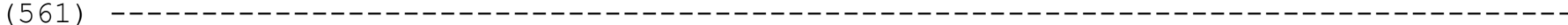

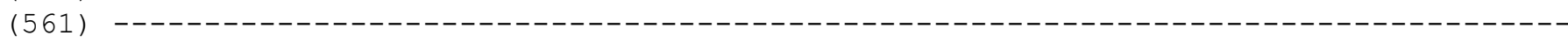

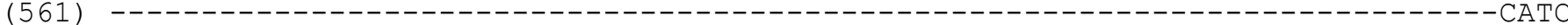

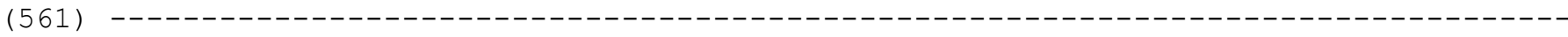

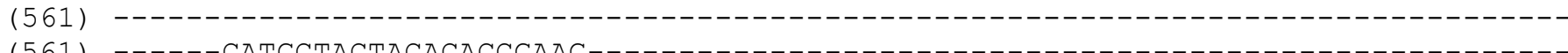

(561) -----CATGCTACTACACACCGAAG-----

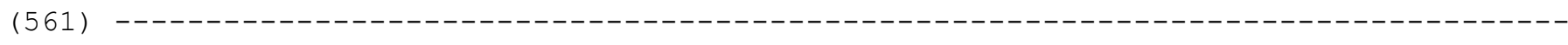




\section{Appendix 3.-Continued}

T. ELEGANS CYTB

T. ATRATUS CYTB

T. SIRTALIS__CYTB

NEFA CYTB

T. GIGAS_CYTB

CYTB forward DW 1

CYTB forward DW 2

T. GIGAS_CYTB_R1

T. GIGAS CYTB R2

T. GIGAS_CYTB_F3

T. GIGAS CYTB Probe3

T. GIGAS_CYTB-R3

T. GIGAS_CYTB_F4

T. ELEGANS CYTB

T. ATRATUS_CYTB

T. SIRTALI $\bar{S}$ CYTB

NEFA_CYTB

T. GI GAS_CYTB

CYTB forward DW 1

CYTB_forward DW 2

T. GIGAS_CYTB R1

T. GIGAS CYTB R2

T. GIGAS_CYTB_F3

T. GIGAS CYTB Probe3

T. GIGAS_CYTB-R3

T. GIGAS_CYTB-F4

T. ELEGANS CYTB

T. ATRATUS CYTB

T. SIRTALIS_CYTB

NEFA CYTB

T. GIGAS CYTB

CYTB_forward DW 1

CYTB forward DW 2

T. GĪGAS_CYTB_R1

T. GIGAS CYTB R2

T. GIGAS_CYTB_F3

T. GIGAS_CYTB_Probe3

T. GIGAS CYTB R3

T. GIGAS_CYTB_F4
641

720

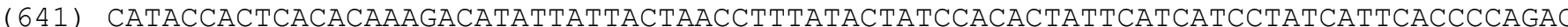

(641) CGTACCACTCACACAAAGACATATTATTACTAACCTTTATACTATCCACACTATTCATCATCCTATCATTCACCCCAGAC

(641) CATACCACTCACATAAAGATATACTACTATTAACCTTCATACTATCCGCACTATTCATCACCCTATCATTCACCCCAGAC

(641) CATACCACTCACACAAAGACATACTACTACTAACCATTATATTATCTGCACTATTCATCATCCTATCATTCACCCCAGAC

(641) CGTACCACTCACACAAAGACATATTATTACTAACCCTTATACTATCCACACTATTCATCATCCTATCATTCACCCCAGAC

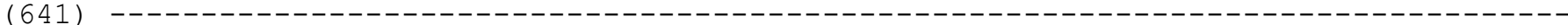

( 641$)-----------------------------------------------------------------------------1$

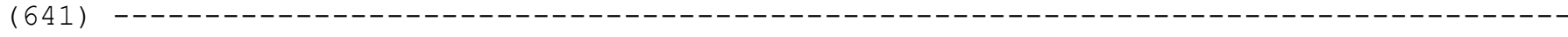

(641) CGTACCACTCACACAAAGACATAT------------------------------------------------

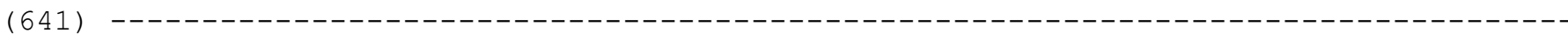

(641) --- - - - - - - - - - - - - - - - - - - - - - - - - - - - - - - - - - - - - - - - - - - - - - - - - - - - - - - - - -

( 641$)-------------------------------------------------------------------------------1$

(641)

721

800

(721) GCATTCAACGACCCAGAAAACTTCTCAAAAGCAAACCCACTAGTAACACCACAACACATTAAACCAGAATGATACTTTCT

(721) GCATTCAACGACCCAGAAAACTTCTCAAAAGCAAACCCACTAGTAACACCACAACACATTAAACCAGAATGATACTTTCT

(721) ATATTCAACGACCCAGAAAACTTCTCAAAAGCAAACCCACTAGTAACACCACAACACATTAAACCAGAATGGTATTTTCT

(721) ATATTCAACGACCCAGAGAACTTCTCAAAAGCAAATCCACTAGTAACACCACAACACATTAAACCAGAGTGATATTTTCT

(721) ACATTCAACGACCCAGAAAACTTCTCAAAAGCAAACCCACTAGTAACACCACAACACATTAAACCAGAATGATACTTTCT

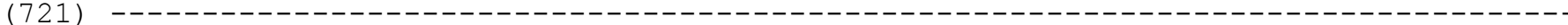

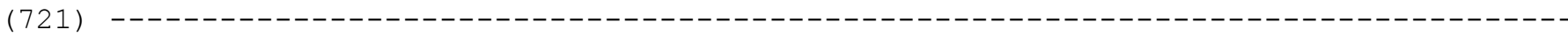

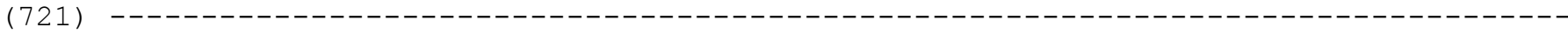

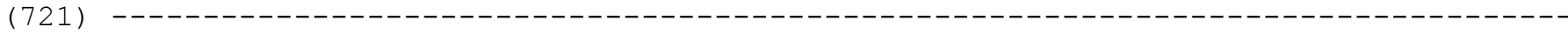

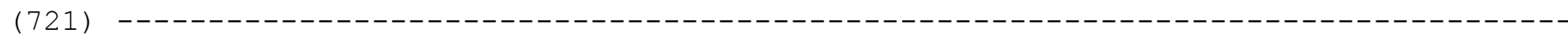

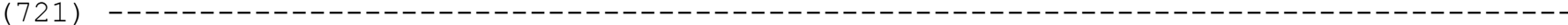

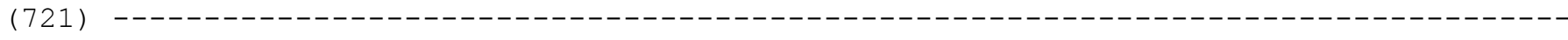

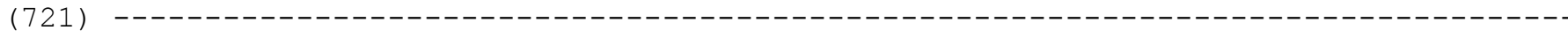
801

880

(801) ATtTGCATATGGCATCCTCCGATCTATCCCTAACAAACTAGGAGGAACAATTGCCCTAGTATTATCAGTTACCATCCTAT

(801) ATtTGCATATGGTATCCTCCGATCTATCCCTAACAAACTAGGAGgAACAATTGCCCTAGTATTATCAATTACCATCCTAT

(801) ATTTGCATATGGTATCCTCCGATCTATCCCCAATAAACTCGGAGGGACGATCGCTCTAGTATTATCAGTCACTATCCTAT

(801) ATTTGCATACGGAATCCTACGATCTATCCCCAACAAACTCGGAGGAACAATCGCCCTAGTATTATCAGTCACCATCCTAT

(801) ATTTGCATATGGCATCCTCCGATCTATCCCCAACAAACTAGGAGGAACAATTGCCCTAGTATTATCAGTTACCATCCTAT

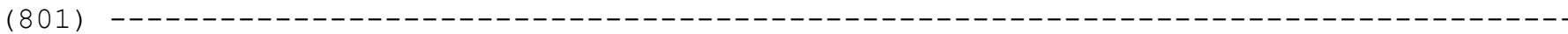

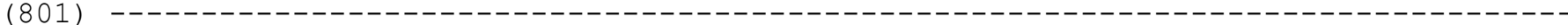

( 801$)--------------------------------------------------------------------------------$

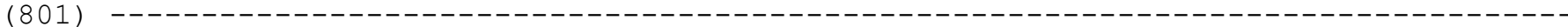

(801) --- - - - - - - - - - - - - - - - - - - - - - - - - - - - - - - - - - - - - - - - - - - - - - - - - - - - - - - - - - - - -

$(801)---------------------------------------------------------------------$

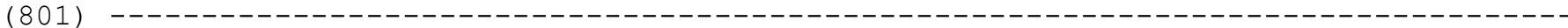

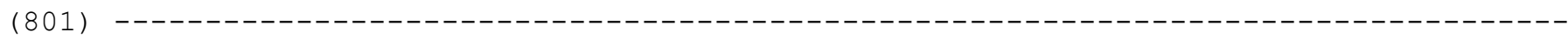




\section{Appendix 3.-Continued}

T. ELEGANS CYTB

T. ATRATUS CYTB

T. SIRTALIS_CYTB

NEFA CYTB

T. GIGAS_CYTB

CYTB forward DW 1

CYTB forward DW 2

T. GIGAS_CYTB_RI

T. GIGAS CYTB R2

T. GIGAS_CYTB_F3

T. GIGAS CYTB Probe 3

T. GIGAS_CYTB-R3

T. GIGAS_CYTB_E4

T. ELEGANS CYTB

T. ATRATUS_CYTB

T. SIRTALI $\bar{S}$ CYTB

NEFA_CYTB

T. GIGAS_CYTB

CYTB forward DW 1

CYTB forward DW 2

T. GIGAS_CYTB R1

T. GIGAS CYTB R2

T. GIGAS_CYTB_F3

T. GIGAS CYTB Probe 3

T. GIGAS_CYTB-R3

T. GIGAS_CYTB-F4

T. ELEGANS CYTB

T. ATRATUS CYTB

T. SIRTALIS_CYTB

NEFA CYTB

T. GIGAS CYTB

CYTB forward DW 1

CYTB forward DW 2

T. GĪGAS_CYTB_R1

T. GIGAS CYTB R2

T. GIGAS_CYTB_F3

T. GIGAS_CYTB_Probe3

T. GIGAS CYTB R3

T. GIGAS_CYTB_F4
881

960

(881) TTACATTACCCTTCACCCATACCTCTCATGTACGATCAATAGCCTTCCGCCCAATAGCACAACTCACATTTTGAATCCTA

(881) TCACATTACCCTTCACCCACACCTCTCATGTACGATCAATAACCTTCCGCCCAATAGCACAACTCACATTTTGAATCCTA

(881) TTACGATACCCTTTACCCACACCTCTCATGTACGATCAATAACTTTCCGCCCAATAGCACAGCTCATGTTTTGAATCCTA

(881) TTACAACACCATTCACCCACACCTCGAATGTGCGGTCAATAACCTTCCGCCCATTAGCACAACTCACATTTTGAATCCTA

(881) TTACATTACCCTTCACCCACACCTCTCATGTACGATCAATAACCTTCCGCCCAATAGCACAACTCACATTTTGAATCCTA

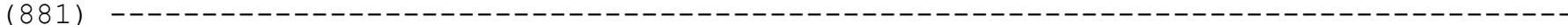

$(881)------------------------------------------------------------------------------$

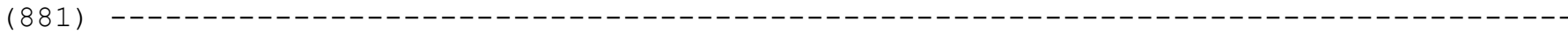

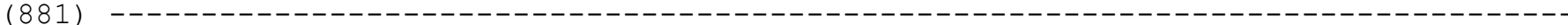

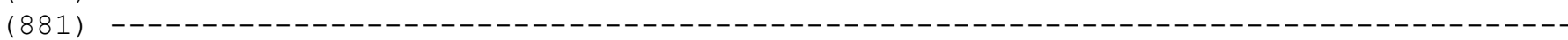

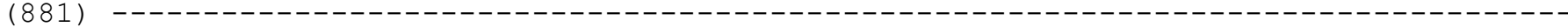

(881) --- - - - - - - - - - - - - - - - - - - - - - - - - - - - - - - - - - - - - - - - - - - - - - - - - - - - - - - - - - - - -

$(881)$

961

1040

(961) ATCGCCACCTTCATCATAATCACATGATCGGCTACAAAACCAGTAGAAACACCATTCACCTTAATCGGCCAAATCACCTC

(961) ATCGCCACCTTCATCATAATCACATGATCAGCTACAAAACCAGTAGAAACACCATTCACCCTAATCGGTCAAATCACCTC

(961) ATTGCCACCTTCATCATAATCACATGATCAGCCACAAAACCAGTAGAAACACCATTCACCATAATTGGCCAAATCACCTC

(961) ATCGCCACCTTCATCATAATCACATGATCGGCTACAAAACCAGTAGAAACACCATTCACCCTAATCGGTCAAATCACCTC

(961) ATCGCCACCTTCATCATAATCACATGATCGGCTACAAAACCAGTAGAAACACCATTCACCCTAATCGGTCAAATCACCTC

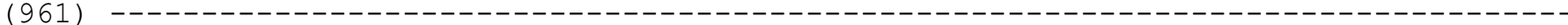

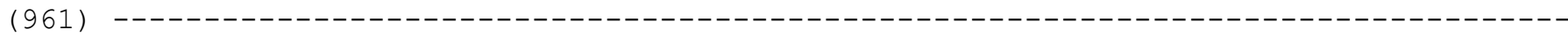

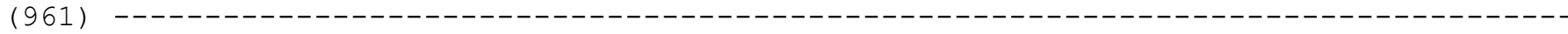

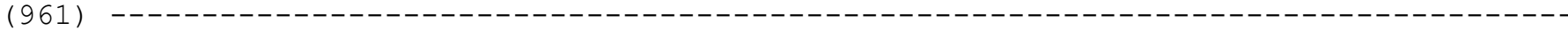

( 961$)$--- - - - - - - - - - - - - - - - - - - - - - - - - - - - - - - - - - - - - - - - - - - - - - - - - - - - - - - - - - -

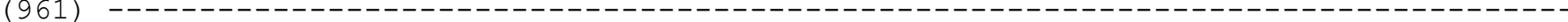

( 961$)$ - - - - - - - - - - - - - - - - - - - - - - - - - - - - - - - - - - - - - - - - - - - - - - - - - - - - - - - - - -

( 961$)$ - - - - - - - - - - - - - - - - - - - - - - - - - - - - - - - - - - - - - - - - - - - - - - - - - - - - -

1041

1117

(1041) TATTTTATACTTCACATTCTTCATAACCACCCCCTTACTAGGTTGATCAGAAAATAAACTTTCAACCACCAACACTT

(1041) TATTTTATACTTCACATTCTTCATAACCACCCCCTTACTAGGTTGATCAGAAAATAAACTTTCAACCACCAACACTT

(1041) TATTTTATACTTCACATTTTCATAATTATCCCTATGTTAGgGTGGTCAGAAAATAAACTTTCAACCACCAACACTT

(1041) TATTTTATACTTCACATTCTTCATAACCACCCCCTTACTAGGTTGATCAGAAAATAAACTTTCAACCACCAACACTT

(1041) TATTTTATACTTCACATTCTTCATAACCACCCCCTTACTAGGTTGATCAGAAAATAAACTTTCAACCACCAACACTT

(1041) --------------------------------------------------------------------------

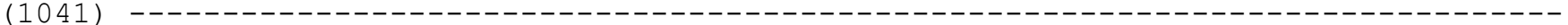

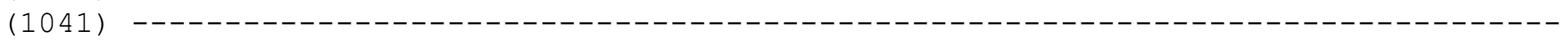

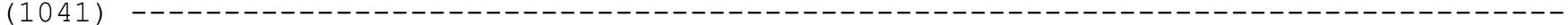

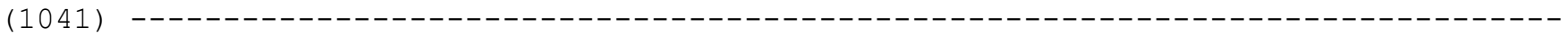

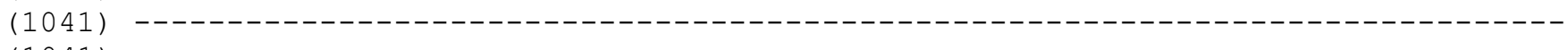

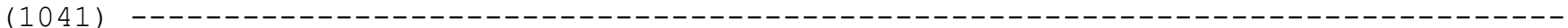

( 1041$)-----------------------------------------------------------------------------$ 
Appendix 4. Sequence Alignment of Thamnophis gigas and Nerodia fasciata and Potentially Sympatric Snake Species for the Nicotinamide Adenine Dinucleotide (Reduced Form) (NADH) Dehydrogenase Subunit 2 (ND2) and Primers/Probe Set for Use in Quantitative Polymerase Chain Reaction (qPCR) Assays, in the Central Valley, California

[Sequences highlighted in blue indicate the conserved nucleotides across the different species' sequence alignments]

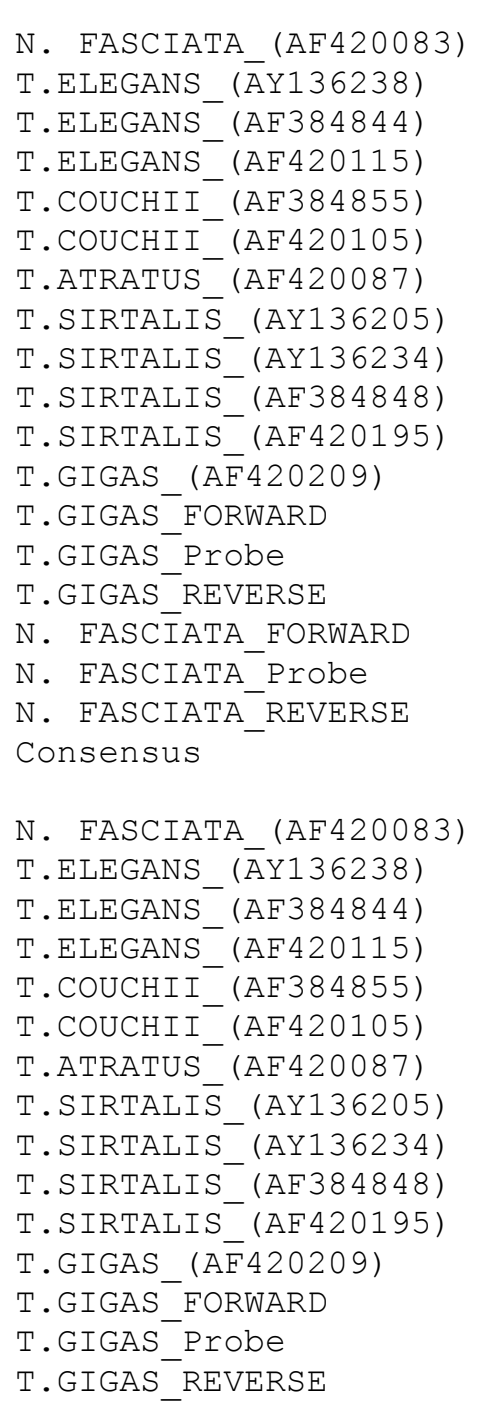

(1) -------ATTAACCTAATATCATGACTTACAATCTCAACAAGCATCATTCTAAGCACAACCATAATTACATCAAC

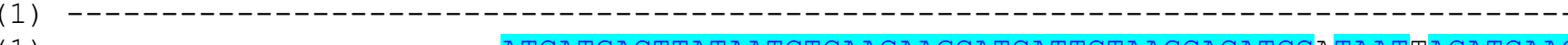

(1) ----------------- ATCATGACTTATAATCTCAACAAGCATCATTCTAAGCACATCCATAATTACATCAAC

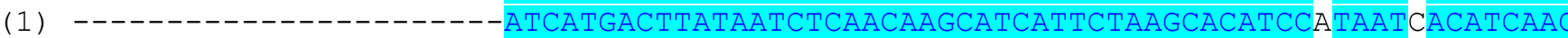

(1) --------ATTAACCTAATATCATGACTTATAATCTCAACAAGCATCATTCTAAGCACATCCCTAATTACATCAAC

(1) ---------ATTAACCTAATATCATGACTTATAATCTCAACAAGCATCATTCTAAGCACATCCATAATTACATCAAC

(1) AgGCCTCTACTAATTAACCTAATATCATGACTTATAATCTCAACAAGCATCATTCTAAGCACATCCGTAATCACATCAAC

(1) -------CTAATTAACCTAATATCATGACTTATAATCTCAACAAGCATCATTCTAAGCACATCCGTAATCACATCAAC

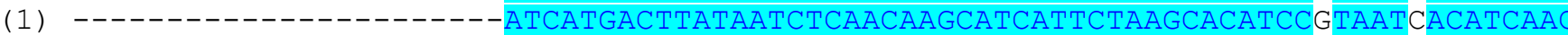

(1) --------ATTAACCTAATATCATGACTTATAATCTCAACAAGCATCATTCTAAGCACATCCGTAATCACATCAAC

(1)

(1)

(1)

(1) -

(1)

(1)

(1)

(1)

ATCATGACTTATAATCTCAACAAGCATCATTCTAAGCACATCC TAAT ACATCAAC 81

(81) AACACATTGATTGATAACCTGAGCATGCCTAGAAATCAACACACTAGCCATAACCCCAATAATCTCCAAACCAAACCACC

(81) -------GATTGATAACCTGAACATGCCTAGAAATTAATACACTATCTATAACCCCTATAATCTCCAAACCCAACCACC

(81) AACACACTGATTGATAACCTGAACATGCCTAGAAATTAATACACTATCTATAACCCCTATAATCTCCAAACCCAACCACD

(81) AACACACTGATTAATAACCTGAGCATGCCTAGAAATCAATACACTATCCATAACCCCTATAATCTCCAAACCCAACCACC

(81) AACACACTGATTAATAACCTGAGCATGCCTAGAAATCAATACACTATCCATAACCCCTATAATCTCCAAACCCAACCACC

(81) AACGCACTGACTGATAACCTGAACATGCCTAGAAATCAATACACTATCCATAACCCCTATAATCTCCAAACCTAACCACC

(81) AACACACTGATTAATAACCTGAGCATGCCTAGAAATCAATACACTATCCATAACCCCTATAATCTCCAAACCCAACCACC

(81) AACACATTGACTGATAACCTGAGCATGCCTAGAAATCAACACACTATCCATAACCCCAATGATCTCTAAACCAAACCACC

(81) AACACATTGACTGATAACCTGAGCATGCCTAGAAATCAACACACTATCCATAACCCCAATGATCTCTAAACCAAACCACC

(81) AACACATTGACTGATAACCTGAGCATGCCTAGAAATCAACACACTATCCATAACCCCAATGATCTCTAAACCAAACCACC

(81) AACACATTGACTGATAACCTGAGCATGCCTAGAAATCAACACACTATCCATAACCCCTATGATCTCTAAACCAAACCACC

(81) AACACACTGACTGATAACCTGAGCATGCCTAGAAATCAATACACTATCCATAACCCCTATAATCTCCAAACCTAACCACC

(81) -

(81)

(81) 


\section{Appendix 4.-Continued}

N. FASCIATA FORWARD

N. FASCIATA - Probe

N. FASCIATA_REVERSE

Consensus

N. FASCIATA (AF420083)

T.ELEGANS_( (A Y136238)

T.ELEGANS_ (AF384844)

T.ELEGANS_(AF420115)

T.COUCHII_(AF384855)

T.COUCHII (AF420105)

T.ATRATUS_(AF420087)

T.SIRTALIS (AY136205)

T.SIRTALIS_(AY136234)

T.SIRTALIS_(AF384848)

T.SIRTALIS (AF420195)

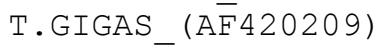

T.GIGAS FORWARD

T.GIGAS_Probe

T.GIGAS_REVERSE

N. FASCIATA FORWARD

N. FASCIATA _ Probe

N. FASCIATA REVERSE

Consensus

N. FASCIATA (AF420083)

T.ELEGANS_( $(\bar{A} Y 136238)$

T.ELEGANS (AF384844)

T.ELEGANS_(AF420115)

T.COUCHII (AF384855)

T.COUCHII (AF420105)

T.ATRATUS_(AF420087)

T.SIRTALIS (AY136205)

T.SIRTALIS_(AY136234)

T.SIRTALIS_(AF384848)

T.SIRTALIS (AF420195)

T.GIGAS_(A $\bar{F} 420209)$

T.GIGAS FORWARD

T.GIGAS_Probe

T.GIGAS REVERSE

N. FASCIATA FORWARD

N. FASCIATA_ Probe
(81)

(81) AACACA TGA TGATAACCTGAgCATGCCTAGAAATCAA ACACTATCCATAACCCC AT ATCTC AAACC AACCACC 161

240

(161) CTCGAGCAACAGAAGCCGCAACAAAATACTACCTCACCCAAACCATCGCCTCTACAACCATACTATTCTCAGCTACAATA

(161) CACGAGCAACAGAAGCCGCAACAAAATATTACCTAACCCAAACCATCGCCTCCACAACCATACTATTCTCAGCCACAATA

(161) CACGAGCAACAGAAGCCGCAACAAAATATTACCTAACCCAAACCATCGCCTCCACAACCATACTATTCTCAGCCACAATA

(161) CACGAGCAACAGAAGCCGCAACAAAATACTACCTAACCCAAACCATCGCCTCCACAACCATACTATTCTCAGCCACAATA

(161) CACGAGCAACAGAAGCCGCAACAAAATACTACCTAACCCAAACCATCGCCTCCACAACCATACTATTCTCAGCCACAATA

(161) CACGAGCAACCGAAGCCGCAACAAAATACTACCTAACCCAAACCATCGCCTCCACAACCATACTATTCTCAGCCACAATG

(161) CACGAGCAACAGAAGCCGCAACAAAATACTACCTAACCCAAACCATCGCCTCCACAACCATACTGTTCTCAGCCACAATA

(161) CACGAGCAACAGAAGCCGCAACAAAATACTACCTAACCCAAACCATCGCCTCCACAACCATACTATTTTCAGCCACAATA

(161) CACGAGCAACAGAAGCCGCAACAAAATACTACCTAACCCAAACCATCGCCTCCACAACCATACTATTTTCAGCCACAATA

(161) CACGAGCAACAGAAGCCGCAACAAAATACTACCTAACCCAAACCATCGCCTCCACAACCATACTATTTTCAGCCACAATA

(161) CACGAGCAACAGAAGCTGCAACAAAATACTACCTAACCCAAACCATCGCCTCCACAACCATACTATTTTCAGCCACAATA

(161) CACGAGCAACCGAAGCCGCAACAAAATACTACCTAACCCAAACCATCGCCTCCACAACCATACTATTCTCAGCCACAATG

(161) -

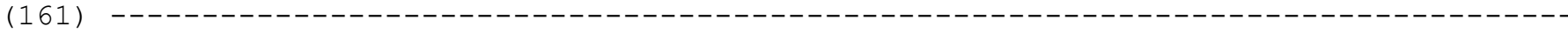

(161) ----------------- - - - - - - - - - - - - - - - - - - - - - - - - - - - - - - - - - - - - - - - - - - -

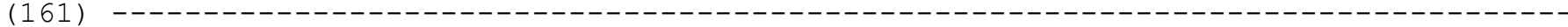

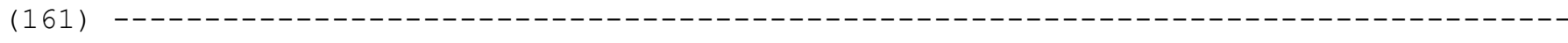

(161)

(161) CACGAGCAACAGAAgCCGCAACAAAATACTACCTAACCCAAACCATCGCCTCCACAACCATACTATT TCAGCCACAATA 241

320

(241) AACGCCCTAАACACCTCAAАCTGAGAAATACACCTCACAACAGAGCCAATAACAАСTATCATCACCCTAGCCCTTATAAT

(241) AACGCCCTAAACACCTCAAACTGAGAAATACACCTCACAATAGAACCGATAACAACCATCATCACCCTAGCCCTTATAAT

(241) AACGCCCTAAACACCTCAAACTGAGAAATACACCTCACAATAGAACCGATAACAACCATCATCACCCTAGCCCTTATAAT

(241) AACGCCCTAAACACCTCAAACTGAGAAATACACCTCACAATAGAACCAATAACAACCATCATCACCCTAGCCCTCATAAT

(241) AACGCCCTAAACACCTCAAACTGAGAAATACACCTCACAATAGAACCAATAACAACCATCATCACCCTAGCCCTCATAAT

(241) AACGCCCTAAACACCTCAAACTGAGAAATACACCTCACAACAGAACCAATAACAACCATCATCACCCTAGCCCTCATAAT

(241) AACGCCCTAAACACCTCAAACTGAGAAATACACCTCACAATAGAACCAATAACAACCATCATCACCCTAGCCCTCATAAT

(241) AACGCCCTAAATACCTCAAACTGAGAAATACACCTCACAATAGAACCAATAACAACCATCATCACCCTAGCCCTCATAAT

(241) AACGCCCTAAACACCTCAAACTGAGAAATACACCTCACAATAGAACCAATAACAACCATCATCACCCTAGCCCTCATAAT

(241) AACGCCCTAAACACCTCAAACTGAGAAATACACCTCACAATAGAACCAATAACAACCATCATCACCCTAGCCCTCATAAT

(241) AACGCCCTAAACACCTCAAACTGAGAAATACACCTCACAATAGAACCAATAACAACCATCATCACCCTAGCCCTCATAAT

(241) AACGCCCTAAACACCTCAAACTGAGAAATACACCTCACAATAGAACCAATAACAACCATCATCACCCTAGCCCTTATAAT

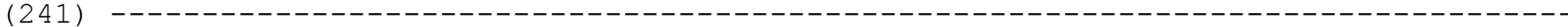

(241) --- - - - - - - - - - - - - - - - - - - - - - - - - - - - - - - - - - - - - - - - - - - - - - - - - - - - - - - - - - - - - - - -

(241) --- - - - - - - - - - - - - - - - - - - - - - - - - - - - - - - - - - - - - - - - - - - - - - - - - - - - - - - - - - - - - - -

(241) -

(241) 


\section{Appendix 4.-Continued}

N. FASCIATA_REVERSE Consensus

N. FASCIATA_(AF420083) T.ELEGANS (ĀY136238)

T.ELEGANS ${ }^{-}$(AF384844)

T.ELEGANS ${ }^{-}$(AF420115)

T.COUCHII (AF384855)

T.COUCHII-(AF420105)

T.ATRATUS (AF420087)

T.SIRTALI $\bar{S}_{-}(A Y 136205)$

T.SIRTALIS ${ }^{-}$(AY136234)

T.SIRTALIS (AF384848)

T.SIRTALIS_(AF420195)

T.GIGAS (A $\bar{F} 420209)$

T.GIGAS_FORWARD

T.GIGAS Probe

T.GIGAS REVERSE

N. FASCĪATA_FORWARD

N. FASCIATA ${ }^{-}$Probe

N. FASCIATA- REVERSE

Consensus

N. FASCIATA_(AF420083)

T.ELEGANS (AY136238)

T.ELEGANS ${ }^{-}$(AF384844)

T.ELEGANS ${ }^{-}$(AF420115)

T.COUCHII (AF384855)

T.COUCHII- (AF420105)

T.ATRATUS (AF420087)

T.SIRTALI $\bar{S}_{-}$(AY136205)

T.SIRTALIS ${ }^{-}$(AY136234)

T.SIRTALIS ${ }^{-}$(AF384848)

T.SIRTALIS_(AF420195)

T.GIGAS (AF 420209$)$

T.GIGAS_FORWARD

T.GIGAS Probe

T.GIGAS REVERSE

N. FASCĪATA_FORWARD

N. FASCIATA Probe

N. FASCIATA_ REVERSE

Consensus
(241)

(241) AACGCCCTAAACACCTCAAACTGAGAAATACACCTCACAATAGAACCAATAACAACCATCATCACCCTAGCCCT ATAAT 321

400

(321) AAAAATGGCAGCAGCACCATTCCACTTCTGACTACCAGAAGTATCACAGGGGGCCACAACAATAACCACCTTAATAATCC

(321) AAAAATGGCAGCAGCACCATTCCACTTCTGACTCCCAGAAGTGTCACAAGGAGCCACAACAATAACCACCCTAACAATCC

(321) AAAAATGGCAGCAGCACCATTCCACTTCTGACTCCCAGAAGTGTCACAAGGAGCCACAACAATAACCACCCTAACAATCC

(321) AAAAATGGCAGCAGCACCTTTCCACTTCTGACTCCCAGAAGTGTCACAAGGAGCCACAACAATAACCACTTTAACAATCC

(321) AAAAATGGCAGCAGCACCTTTCCACTTCTGACTCCCAGAAGTGTCACAAGGAGCCACAACAATAACCACTTTAACAATCC

(321) AAAAATGGCAGCAGCACCTTTCCACTTCTGACTCCCAGAAGTGTCACAAGGAGCCACAACAATAACCACTTTAACAATCC

(321) AAAAATGGCAGCAGCACCTTTCCACTTCTGACTCCCAGAAGTGTCACAAGGAGCCACAACAATAACCACTTTAACAATCC

(321) AAAAATGGCAGCAGCACCATTCCACTTTTGACTCCCAGAAGTATCACAAGGGACCACAACAATAACCACTCTAACAATCC

(321) AAAAATGGCAGCAGCACCATTCCACTTTTGACTCCCAGAAGTATCACAAGGGACCACAACAATAACCACTCTAACAATCC

(321) AAAAATGGCAGCAGCACCATTCCACTTTTGACTCCCAGAAGTATCACAAGGGACCACAACAATAACCACTCTAACAATCC

(321) AAAAATGGCAGCAGCACCATTCCACTTTTGACTCCCAGAAGTATCACAAGGGACCACAACAATAACCACTCTAACAATCC

(321) AAAAATGGCAGCAGCACCTTTCCACTTCTGACTTCCAGAAGTGTCACAAGGAGCCACAACAATAACCACTTTAACAATCC

(321)

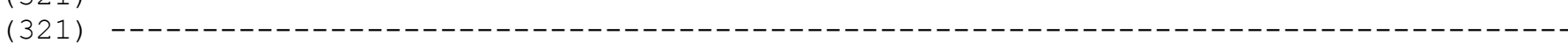

(321) - - - - - - - - - - - - - - - - - - - - - - - - - - - - - - - - - - - - - - - - - - - - - - - - - - - -

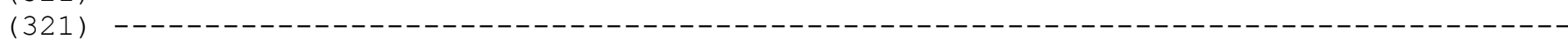

(321) --- - - - - - -

$(321)$

(321) AAAAATGGCAGCAGCACC TTCCACTT TGACTCCCAGAAGT TCACAAGG CCACAACAATAACCACT TAACAATCC 401

480

(401) TCACATGACAAAAAATCGCACCACTCACAATCCTCCTTAACACCAACAACAAAATCAACACCTCACTTATCCTATCATGC

(401) TCACATGACAAAAAATCGCACCACTCACAATCCTACTCAATACCAACAACAAAATCAACACCTCACTTATACTATTATGC

(401) TCACATGACAAAAAATCGCACCACTCACAATCCTACTCAATACCAACAACAAAATCAACACCTCGCTTATACTACTGAGC

( 401 ) TCACATGACAAAAAATCGCACCACTCACAATCCTACTTAATACCAACAACAAAATCAACACCTCACTTATACTACTGAGC

( 401 ) TCACATGACAAAAAATCGCACCACTCACAATCCTACTTAATACCAACAACAAAATCAACACCTCACTTATACTACTGAGC

(401) TCACATGACAAAAAATCGCACCACTCACAATCTTACTTAATACCAACAACAAAATCAACACCTCACTTATACTACTGAGC

(401) TCACATGACAAAAAATCGCACCGCTCACAATCCTACTTAATACCAACAACAAAATCAACACCTCACTTATACTACTAAGC

(401) TCACATGACAAAAAATCGCACCACTCACAATCCTACTCAACACCAACAACAAAATCAACACCTCCCTCATCCTATTATGC

( 401 ) TCACATGACAAAAAATCGCACCACTCACAATCCTACTCAACACCAACAACAAAATCAACACCTCCCTCATCCTATTAAGC

( 401 ) TCACATGACAAAAAATCGCACCACTCACAATCCTACTCAACACCAACAACAAAATCAACACCTCCCTCATCCTATTAAGC

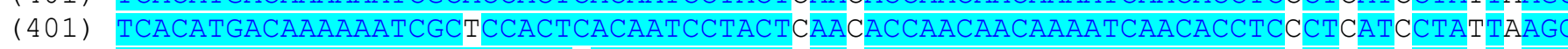

(401) TCACATGACAAAAAATCGCACCACTTACAATCCTACTTAACACCAACAACAAAATCAACACCTCACTTATACTACTGAGC

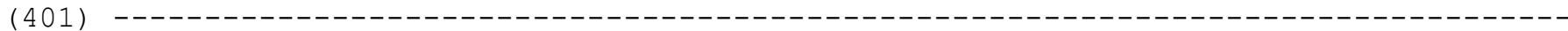

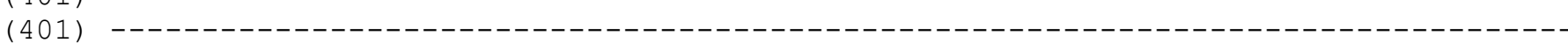

( 401$)$ - - - - - - - - - - - - - - - - - - - - - - - - - - - - - - - - - - - - - - - - - - - - - - - - - - - - - - -

$(401)$ - - - - - - - - - - - - - - - - - - - - - - - - - - - - - - - - - - - - - - - - - - - - - - - - - - - - - - - - - - - - -

( 401$)$ - - - - - - - - - - - - - - - - - - - - - - - - - - - - - - - - - - - - - - - - - - - - - - - - - - - - - - - -

( 401 ) --------------------------------------------------------------------------

(401) TCACATGACAAAAAATCGCACCACTCACAATCCTACT AA ACCAACAACAAAATCAACACCTC CT AT CTA T AGC 


\section{Appendix 4.-Continued}

N. FASCIATA (AF420083) T.ELEGANS_( (A $Y 136238)$ T.ELEGANS (AF384844) T.ELEGANS (AF420115) T.COUCHII_(AF384855) T.COUCHII (AF420105) T.ATRATUS_ (AF420087) T.SIRTALIS (AY136205) T.SIRTALIS (AY136234) T.SIRTALIS_(AF384848) T.SIRTALIS (AF420195)

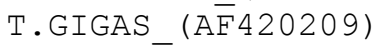
T.GIGAS FORWARD

T.GIGAS Probe T.GIGAS REVERSE N. FASCIATA FORWARD N. FASCIATA_ Probe N. FASCIATA REVERSE Consensus

N. FASCIATA (AF420083) T.ELEGANS_( $(\bar{A} Y 136238)$ T.ELEGANS ${ }^{-}$(AF384844)

T.ELEGANS (AF420115) T.COUCHII_(AF384855) T.COUCHII (AF420105) T.ATRATUS - (AF420087) T.SIRTALIS (AY136205) T.SIRTALIS_(AY136234) T.SIRTALIS (AF384848) T.SIRTALIS (AF420195) T.GIGAS_(A $\bar{F} 420209)$ T.GIGAS FORWARD

T.GIGAS_Probe T.GIGAS_REVERSE N. FASCIATA FORWARD N. FASCIATA_ Probe N. FASCIATA REVERSE Consensus
481

560

(481) GCCACCCTATCAATCATCCTTGGGGGCCTTGGAGGCCTAAACCAAACTCAACTACGAAAACTTATAGCATTCTCATCAAT (481) GCCACCCTATCAATCATCATCGGAGGTCTCGGAGGCCTCAACCAAACCCAACTACGAAAACTAATAGCATTCTCATCAAT (481) GCCACCCTATCAATCATCATCGGAGGTCTCGGAGGCCTCAACCAAACCCAACTACGAAAACTAATAGCATTCTCATCAAT (481) GCCACCTTATCAATCATCATCGGAGGTCTCGGAGGCCTCAACCAAACCCAACTACGAAAACTAATAGCATTCTCATCAAT (481) GCCACCTTATCAATCATCATCGGAGGTCTCGGAGGCCTCAACCAAACCCAACTACGAAAACTAATAGCATTCTCATCAAT (481) GCCACCTTATCAATCATCATCGGAGGCCTCGGAGGCCTCAACCAAACCCAACTACGAAAACTAATAGCATTCTCATCAAT (481) GCCACCCTATCAATCATCATCGGAGGTCTCGGAGGCCTCAACCAAACCCAACTACGAAAACTAATAGCATTCTCATCAAT (481) GCCACCCTATCAATCATCATTGGAGGCCTAGGAGGCCTCAACCAAACTCAGCTACGAAAACTAATAGCATTCTCATCAAT (481) GCCACCCTATCAATCATCATTGGAGGCCTAGGAGGCCTCAACCAAACCCAGCTACGAAAACTAATAGCATTCTCATCAAT (481) GCCACCCTATCAATCATCATTGGAGGCCTAGGAGGCCTCAACCAAACCCAGCTACGAAAACTAATAGCATTCTCATCAAT (481) GCCACCCTATCAATCATCATTGGAGGCCTAGGAGGCCTCAACCAAACTCAGCTACGAAAACTAATAGCATTCTCATCAAT (481) GCCACCTTATCAATCATCATCGGAGGTCTCGGAGGCCTCAACCAAACCCAACTACGAAAACTAATAGCATTCTCATCAAT (481) -----

(481)

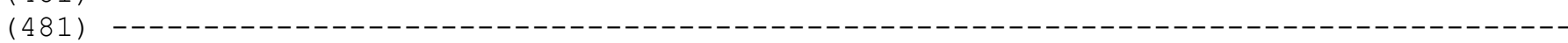

(481) -

(481) -

$(481)$

(481) GCCACC TAtCAATCATCAT GGAgG CT GGAgGCCTCAACCAAACCCA CTACGAAAACTAATAgCAtTCtCAtCAAT 561

640

(561) CGCCCACACAGGATGAATCCTAGCAACACTAAAAATAGCCCCAAACATTTCACTTCTAACCTTTTTAATCTATATTATGA (561) CGCCCATACAGGCTGAATTCTAGCAACACTAAAAATAGCACCGAACATCTCACTACTGACCTTTTTAATCTACATTATAA (561) CGCCCATACAGGCTGAATTCTAGCAACACTAAAAATAGCACCGAACATCTCACTACTGACCTTTTTAATCTACATTATAA (561) CGCCCATACAGGCTGAATTCTAGCAACACTAAAAATAGCACCGAACATCTCACTACTGACCTTTATAATCTACATTATAA (561) CGCCCATACAGGCTGAATTCTAGCAACACTAAAAATAGCACCGAACATCTCACTACTGACCTTTATAATTTACATTATAA (561) CGCCCATACAGGCTGAATTCTAGCAACACTAAAAATAGCACCGAACATCTCACTACTAACCTTTATAATCTACATTATAA (561) CGCCCATACGGGCTGAATTCTAGCAACACTAAAAATAGCACCGAACATCTCACTACTGACCTTTATAATCTACATTATAA (561) TGCCCACACAGGTTGAATCCTAGCAACACTAAAAATAGCACCGAACATCACACTACTCACCTTTATAATCTATATTATAA (561) CGCCCACACAGGTTGAATCCTAGCAACACTAAAAATAGCACCGAACATCTCACTACTCACCTTTATAATCTATATTATAA (561) CGCCCACACAGGTTGAATCCTAGCAACACTAAAAATAGCACCGAACATCTCACTACTCACCTTTATAATCTATATTATAA (561) CGCCCACACAGGTTGAATCCTAGCAACACTAAAAATAGCACCGAACATCTCACTACTCACCTTTATAATCTATATTATAA (561) CGCCCATACAGGCTGAATTCTAGCAACACTAAAAATAGCACCAAACATCTCACTACTCACCTTTATAATCTACATTATAA (561) -

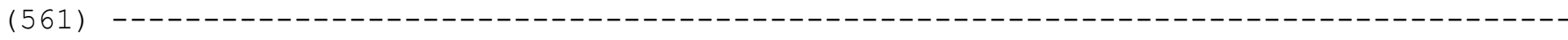

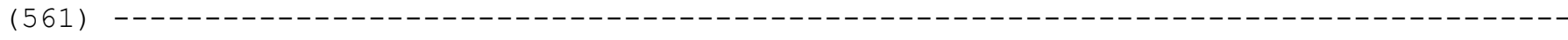

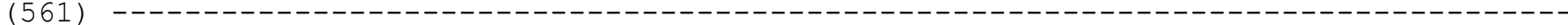

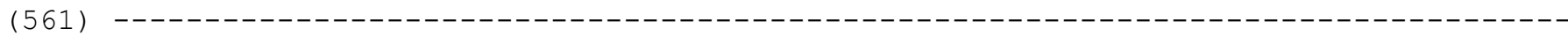

$(561)$

(561) CGCCCA ACAGG tGAAT CTAGCAACACTAAAAATAGCACCGAACATCTCACTACT ACCTTtATAATCTA ATtATAA 


\section{Appendix 4.-Continued}

N. FASCIATA (AF420083) T.ELEGANS_( (A $Y 136238)$ T.ELEGANS (AF384844) T.ELEGANS (AF420115) T.COUCHII_(AF384855) T.COUCHII (AF420105) T.ATRATUS_ (AF420087) T.SIRTALIS (AY136205) T.SIRTALIS (AY136234) T.SIRTALIS_(AF384848) T.SIRTALIS (AF420195)

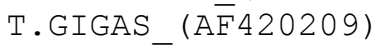
T.GIGAS FORWARD

T.GIGAS Probe T.GIGAS REVERSE N. FASCIATA FORWARD N. FASCIATA_ Probe N. FASCIATA REVERSE Consensus

N. FASCIATA (AF420083) T.ELEGANS_( $(\bar{A} Y 136238)$ T.ELEGANS $($ AF384844) T.ELEGANS_(AF420115) T.COUCHII (AF384855) T.COUCHII (AF420105) T.ATRATUS - (AF420087) T.SIRTALIS T.SIRTALIS_(AY136234) T.SIRTALIS (AF384848) T.SIRTALIS (AF420195) T.GIGAS_(A $\bar{F} 420209)$ T.GIGAS FORWARD

T.GIGAS_Probe T.GIGAS_REVERSE N. FASCIATA FORWARD N. FASCIATA N. FASCIATA REVERSE Consensus
641

720

(641) CTACCACCCCAATCTTCCTAACCATAAACACCACCACATCAACCACAATAAAAGATATTGGAACAGCTTGACCCTCTTCA (641) CCACAACCCCAATTTTCCTAACCATAAACATCACCACATCAACCACAATAAAAGACATTGGAACAGCTTGACCATCTTCA (641) CCACAACCCCAATTTTCCTAACCATAAACATCACCACATCAACCACAATAAAAGACATTGGAACAGCTTGACCATCTTCA

(641) CCACAACCCCAATTTTCCTAACTATAAACATCACCGCATCAACCACAATAAAAGACATTGGAACAGCCTGACCATCTTCA

(641) CCACAACCCCAATTTTCCTAACTATAAACATCACCGCATCAACCACAATAAAAGACATT GGAACAGCCTGACCATCTTCA

(641) CCACAACCCCAATTTTCCTAACTATAAACATCACCGCATCAACCACAATAAAAGACATTGGAACAGCCTGACCATCTTCA

(641) CCACAACCCCAATTTTCCTAACTATAAACATCACCGTATCAACCACAATAAAAGACATTGGAACAGCCTGACCATCTTCA

(641) CCACAACCCCAATCTTCCTAACCATAAACACCACCTCATCAACCACAATAAAAGACATCGGAACAACTTGACCCTCTTCA

(641) CCACAACCCCAATCTTCCTAACCATAAACACCACCTCATCAACCACAATAAAAGACATCGGAACAACTTGACCCTCTTCA

(641) CCACAACCCCAATCTTCCTAACCATAAACACCACCTCATCAACCACAATAAAAGACATCGGAACAACTTGACCCTCTTCA

(641) CCACAACCCCAATCTTCCTAACCATAAACACCACCTCATCAACCACAATAAAAGACATCGGAACAACTTGACCCTCTTCA (641) CCACGACCCCAATTTTCCTAACTATAAACATCACCGCATCAACTACAATAAAAGACATTGGAACAGCCTGACCATCTTCA (641) -----

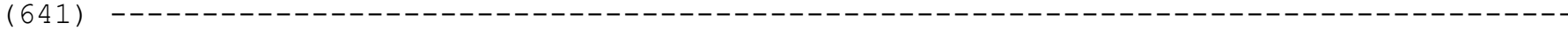

$(641)-------------------------------------------------------------------------$

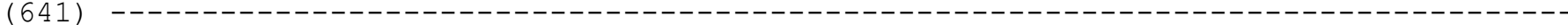

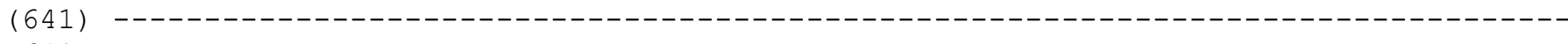

$(641)$

(641) CCACAACCCCAAT tTCCTAAC ATAAACA CACC CATCAACCACAATAAAAGACAT GGAACA C TGACC TCTTCA 721

800

(721) CCTGCTTTAATACTACTACTCTCAACAACCATCCTATCAATGGGCGGCCTACCCCCAATAACAGGATTCATGCCTAAATG

(721) CCGGCCCTTATGTTACTCCTCTCTATAACTATCCTATCAATAGGGGGCCTACCCCCAATAACAGGATTTATACCCAAATG

(721) CCGGCCCTTATGTTACTCCTCTCTATAACTATCCTATCAATAGGgGGCCTACCCCCAATAACAGGATTTATACCCAAATG

(721) CCAGCCCTAATATTGCTTCTTTCTACAGCTATCCTATCAATAGGAGGCCTACCCCCAATAACAGGATTTATACCTAAATG

(721) CCAGCCCTAATATTGCTTCTTTCTACAGCTATCCTATCAATAGGgGGTCTACCCCCAATAACAGGATTTATACCTAAATG

(721) CCAGCCCTTATATTGCTTCTTTCTACAACTATCCTATCAATAGGgGGCCTACCCCCAATAACAGGATTCATACCTAAATG

(721) CCAGCCCTTATATTACTTCTTTCTACAACTATCCTATCAATAGGAGGCCTACCCCCAATAACAGGATTTATACCTAAATG

(721) CCAACСTTAATATTACTACTTTCTACAACCATCCTTTCAATAGGAGGCCTACCCCCAATAACAGGATTCATACCTAAATG

(721) CCAACCTTAATATTACTACTTTCTACAACCATCCTTTCAATAGGAGGCCTACCCCCAATAACAGGATTTATACCTAAATG

(721) CCAACCTTAATATTACTACTTTCTACAACCATCCTTTCAATAGGAGGCCTACCCCCAATAACAGGATTTATACCTAAATG

(721) CCAACCTTAATATTACTACTTTCTACAACCATCCTTTCAATAGGAGGCCTACCCCCAATAACAGGATTTATACCTAAATG

(721) CCAGCCCTTATACTACTTCTTTCTACAACTATCCTATCAATAGGAGGCCTACCCCCAATAACAGGATTTATACCTAAATG

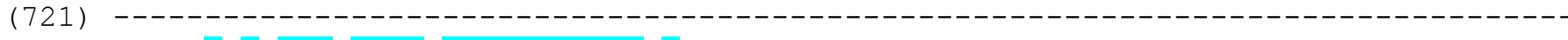

(721) -----CCTTATACTACTTCTTTCTACAACTA-----------------------------------------------

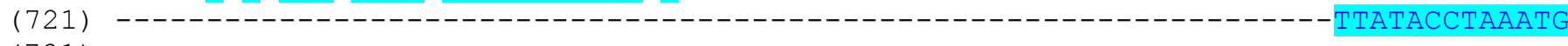

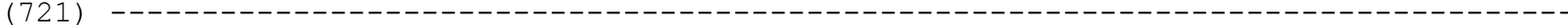

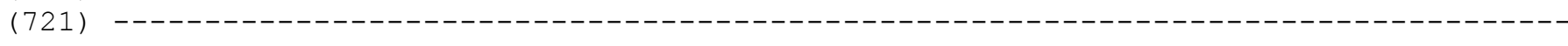

(721)

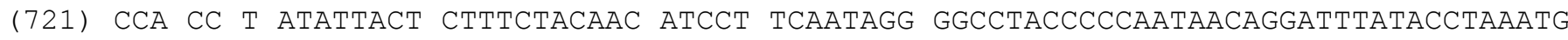




\section{Appendix 4.-Continued}

N. FASCIATA (AF420083) T.ELEGANS_( $\bar{A} Y 136238)$

T.ELEGANS ${ }^{-}$(AF384844)

T.ELEGANS ${ }^{-}$(AF420115)

T.COUCHII-(AF384855)

T.COUCHII (AF420105)

T.ATRATUS_-(AF420087)

T.SIRTALIS_ (AY136205)

T.SIRTALIS ${ }^{-}$(AY136234)

T.SIRTALIS_(AF384848)

T.SIRTALIS (AF420195)

T.GIGAS_(A $\bar{F} 420209$ )

T.GIGAS FORWARD

T.GIGAS Probe

T.GIGAS REVERSE

N. FASCIATA FORWARD

N. FASCIATA - Probe

N. FASCIATA- REVERSE

Consensus

N. FASCIATA (AF420083)

T.ELEGANS_( $\bar{A} Y 136238)$

T.ELEGANS ${ }^{-}$(AF384844)

T.ELEGANS_(AF420115)

T.COUCHII-(AF384855)

T.COUCHII (AF420105)

T.ATRATUS ${ }^{-}$(AF420087)

T.SIRTALI $\bar{S}$ (AY136205)

T.SIRTALIS_(AY136234)

T.SIRTALIS_(AF384848)

T.SIRTALIS (AF420195)

T.GIGAS_(A $\bar{F} 420209)$

T.GIGAS FORWARD

T.GIGAS_Probe

T.GIGAS REVERSE

N. FASCIATA FORWARD

N. FASCIATA- Probe

N. FASCIATA REVERSE

Consensus
801

880

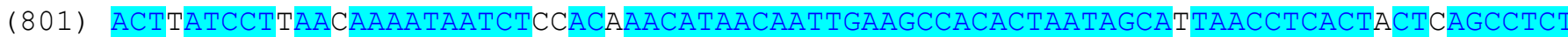

(801) ACTTATCCTAAACAAAATAATCTACACAAACATAACAATTGAAGCCACACTAATAGCACTAACCTCACTACTTAGCCTTT

(801) ACTTATCCTAAACAAAATAATCTACACAAACATAACAATTGAAGCCACACTAATAGCACTAACCTCACTACTTAGCCTTT

(801) ACTCATCCTAAACAAAATAATCTACACGAACATAACAATTGAAGCCACACTAATAGCGCTAACCTCACTGCTTAGCCTCT

(801) ACTCATCCTAAACAAAATAATCTACACGAACATAACAATTGAAGCCACACTAATAGCGCTAACCTCACTGCTTAGCCTCT

(801) ACTCATCCTAAACAAAATAATCTACACAAACATAACAATTGAAGCCACACTAATAGCACTAACCTCACTGCTTATCCTCT

(801) ACTCATCCTAAACAAAATAATCTACACGAACATAACAATTGAAGCCACACTAATAGCGCTAACCTCACTGCTTAGCCTCT

(801) ACTCATCCTAAATAAAATAATCTATACAAACATAACAATTGAAGCCACACTGATGGCATTAACTTCACTACTCAGCCTCT

(801) ACTCATCCTAAATAAAATAATCTATACAAACATAACAATTGAAGCCACACTGATGGCATTAACTTCACTACTCAGCCTCT

(801) ACTCATCCTAAATAAAATAATCTATACAAACATAACAATTGAAGCCACACTGATGGCATTAACTTCACTACTCAGCCTCT

(801) ACTCATCCTAAATAAAATAATCTGTACNAACATAACAATTGAAGCCACACTGATGGCATTAACTTCACTACTCAGCCTCT

(801) ACTCATCCTAAACAAAATAATCTACACAAACATAACAATTGAAGCCACACTAATAGCGCTAACCTCACTGCTTAGCCTCT

$(801)$

$(801)$

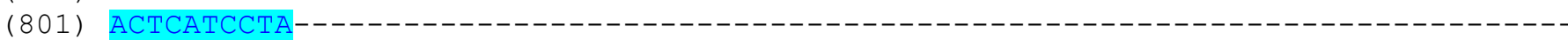

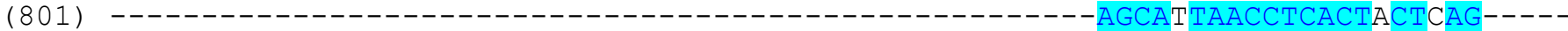

( 801$)----------------------------------------------------------------------------1$

$(801)$

CACACAATAGTGGCCTCAAC

(801) ACTCATCCTAAA AAAATAATCTA AC AACATAACAATTGAAGCCACACTAATAGCA TAACCTCACT CT AGCCTCT 881

960

(881) ACGTATATATACGATTAATATACATATCATCAATAACAACCCCACCACACACCACACTCACACCAATAAAATGACGAACC

(881) ACATTTACATACGACTAATATACGTATCATCAATAACAACCCCACCTCACACTACACTAACACCAATAAAATGACGGACC

(881) ACATTTACATACGACTAATATACGTATCATCAATAACAACCCCACCTCACACTACACTAACACCAATAAAATGACGGACC

(881) ACGTTTACATACGACTAATATACATGTCATCAATAACAACCCCACCCCACACCACACTAACACCAATAAAATGACGAACC

(881) ACGTTTACATACGACTAATATACATGTCATCAATAACAACCCCACCCCACACTACACTAACACCAATAAAATGACGAACC

(881) ACGTTTACATACGACTAATATACATATCATCAATAACAACCCCACCACACACCACACTAACACCAATAAAATGACGAACC

(881) ACGTTTACATACGACTAATATACATGTCATCAATAACAACCCCACCACACACCACACTAACACCAATAAAATGACGAACC

(881) ACGTATATATACGACTTATATACATATCATCAATAACAACCCCCCCACACACTACACTCGCACCAATAAAATGACGAACC

(881) ACGTATATATACGACTTATATACATATCATCAATAACAACCCCCCCACACACTACACTCGCACCAATAAAATGACGAACC

(881) ACGTATATATACGACTTATATACATATCATCAATAACAACCCCCCCACACACTACACTCGCACCAATAAAATGACGAACC

(881) ACGTATATATACGACTTATATACATATCATCAATAACAACCCCCCCACACACTACACTCGCACCAATAAAATGACGAACC (881) ACGTTTACATACGACTAATATACATATCATCAATGACAACCCCACCACACACCACACTAACACCAATAAAATGACGAACC

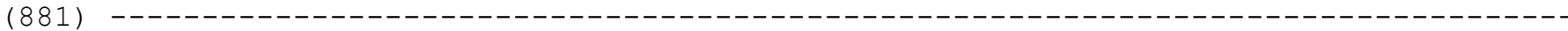

$(881)-------------------------------------------------------------------------$

$(881)-----------------------------------------------------------------------$

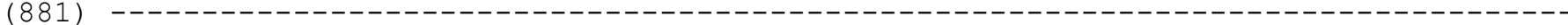

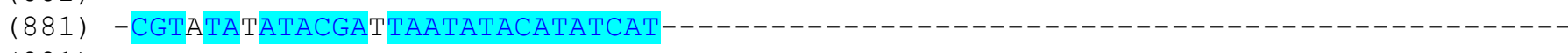

$(881)$

(881) ACGT tA AtACGACtAAtAtACAtAtCAtCAAtAACAACCCC CC CACAC ACACt CACCAAtAAAAtgACGAACC 


\section{Appendix 4.-Continued}

N. FASCIATA (AF420083) T.ELEGANS_( $(\overline{\mathrm{A}} Y 136238)$

T.ELEGANS ${ }^{-}$(AF384844)

T.ELEGANS (AF420115)

T.COUCHII_(AF384855)

T.COUCHII (AF420105)

T.ATRATUS (AF420087)

T.SIRTALIS (AY136205)

T.SIRTALIS (AY136234)

T.SIRTALIS_(AF384848)

T.SIRTALIS (AF420195)

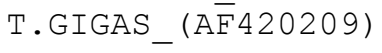

T.GIGAS FORWARD

T.GIGAS Probe

T.GIGAS REVERSE

N. FASCIATA FORWARD

N. FASCIATA - Probe

N. FASCIATA REVERSE

Consensus
961

1040

(961) ACACAAAAAAAACACACAATAGTGGCCTCAACACTAACAATAATAACCTCCCTCCTACTACCACTAACACCAAACATATA

(961) ACACATAAAAATCACACAATGACAACCTCAACATTAACAATAATAACCGCCCTCCTACTTCCACTAACACCTAACATATA

(961) ACACATAAAAATCACACAATGACAACCTCAACACTAACAATAATAACCGCCCTCCTACTTCCACTAACACCTAACAT---

(961) CCGCATAAAAATCACATAATGACAACTTCAACACTAACAATAATAACCACCCTCCTACTCCCACTAACACCTAACATATA

(961) CCACATAAAAATCACATAATGACAACTTCAACACTAACAATAATAACCACCCTCCTACTCCCACTAACACCTAACAT---

(961) ACACATAAAAATCACACAATGACAACTTCAACACTAACAATAATAACCACCTTCCTACTCCCACTWACACCCAACATATA

(961) CCACATAAAAACCACATAATGACAACTTCAACACTAACAATAATAACCACCCTCCTACTCCCACTAACACCTAACATATA

(961) ACACATAAAAAACACACAATGGTAGCTTCAACACTAACAATAATAACCACCCTCCTACTCCCACTTACACCAAATATATA

(961) ACACATAAAAAACACACAATGGTAGCTTCAACACTAACAATAATAACCACCCTCCTACTCCCACTTACACCAAATATATA

(961) ACACATAAAAAACACACAATGGTAGCTTCAACACTAACAATAATAACCACCCTCCTACTCCCACTTACACCAAATAT---

(961) ACACATAAAAAACACACAATGGTAGCTTCAACACTAACAATAATAACCACCCTCCTACTCCCACTTACACCAAATATATA

(961) ACACATAAAAATCACATAATGACAACTTCAACACTAACAATAATAACCACCCTCCTACTCCCATTAACACCTAAGA----

(961)

(961) -

(961) -

(961) -

(961)

(961)

(961) ACACATAAAAA CACA AATG A CTTCAACACTAACAATAATAACCACCCTCCTACTCCCACT ACACC AA AT 

Publishing support provided by the U.S. Geological Survey

Science Publishing Network, Tacoma Publishing Service Center

For more information concerning the research in this report, contact the Director, Western Ecological Research Center

U.S. Geological Survey

3020 State University Drive East

Sacramento, California 95819

http://www.werc.usgs.gov/ 
